\title{
Acid-Base Reactions in Benzene and Other Organic Solvents: Behavior of Bromphthalein Magenta with Different Classes of Organic Bases
}

\author{
By Marion Maclean Davis and Priscilla J. Schuhmann
}

\begin{abstract}
Practical and theoretical aspects of acid-base reactions in benzene and other organic solvents are discussed. The synthesis of the indicators bromphthalein magenta $\mathrm{E}$ and $\mathrm{B}$ (the ethyl and $n$-butyl esters of tetrabromophenolphthalein) and of their tetraalkylammonium salts is described. Qualitative and spectrophotometric data concerning the color reactions of the indicators with primary, secondary, and tertiary aliphatic amines, substituted guanidines, and other nitrogen bases in benzene and other types of organic solvents are presented. An explanation offered for the color phenomena is also applicable to sulfonephthaleins and other indicators.

Examples are given of the quantitative measurement of the relative reactivity of bases with bromphthalein magenta in benzene. Illustrations are furnished of the application of the indicators to the quantitative estimation of acids and bases, to measurements of the relative strengths of organic acids, and to the differentiation of primary, secondary, and tertiary aliphatic amines.

During the spectrophotometric investigations, it was necessary to design new types of absorption cells suitable for volatile solvents and for thin layers of solution. A description of these cells is given.
\end{abstract}

\section{Introduction}

Practical methods for measuring acidity and basicity in organic media have not kept pace with scientific and industrial needs. Moreover, the fundamental principles governing acid-base reactions in the various types of organic solvents are less understood than the principles governing these reactions in aqueous media and are considerably less familiar to the average chemist. This is understandable, as the great variety of organic solvents now commercially available at moderate cost were for the most part chemical curiosities or completely unknown before World War I. As a result, there has been an overemphasis of certain concepts that were developed in the study of aqueous systems. In particular, the theory that acidic and basic behavior are due, respectively, to hydrogen and hydroxyl ions has been firmly implanted in the minds of most chemists who received their scientific training prior to the last 10 or 20 years. Since then, the more general Brönsted-Lowry concept of acids and bases has gained increasing acceptance.
Because an understanding of the work described in this paper requires some familiarity with this theory, a brief explanation will be presented.

The Brönsted-Lowry theory of acidity and basicity.-According to the Brönsted-Lowry theory [1 to 5$]^{1}$ an acid is defined as a proton donor and a base is defined as a proton acceptor, as indicated in the following relationship:

$$
\begin{aligned}
& \mathrm{A} \rightleftarrows \mathrm{B}+\mathrm{H}^{+} . \\
& \text {acid base }
\end{aligned}
$$

Thus, when a compound behaves as an acid it is converted by loss of a proton into a base and, conversely, a base upon combination with a proton becomes an acid. A substance that acts as a base does so because it possesses a pair of unshared electrons. In other words, a base is an electron donor and an acid is an electron acceptor. Later it will be seen that this definition leads to a still more general concept of acids and bases.

Either the acid or the base may be a cation, an

\footnotetext{
${ }^{1}$ Figures in brackets indicate the literature references at the end of this paper.
} 
anion, or an electrically neutral molecule, as shown in the following examples:

$$
\begin{gathered}
\mathrm{NH}_{4}{ }^{+} \rightleftharpoons \mathrm{H}^{+}+\mathrm{NH}_{3} \\
\mathrm{HC}_{2} \mathrm{H}_{3} \mathrm{O}_{2} \rightleftharpoons \mathrm{H}^{+}+\mathrm{C}_{2} \mathrm{H}_{3} \mathrm{O}_{2}^{-} \\
\mathrm{H}_{2} \mathrm{O} \rightleftharpoons \mathrm{H}^{+}+\mathrm{OH}^{-} \\
\mathrm{HSO}_{4}^{-} \rightleftharpoons \mathrm{H}^{+}+\underset{\mathrm{SO}_{4}=}{\text { bases }} \\
\text { acids }
\end{gathered}
$$

Water behaves not only as an acid!but also as a base, as shown in the following equation:

$$
\underset{\text { base }}{\mathrm{H}_{2} \mathrm{O}}+\mathrm{H}^{+} \underset{\text { acid }}{\rightleftharpoons} \mathrm{H}_{3} \mathrm{O}^{+}
$$

It is now generally accepted that the hydrogen ion does not exist in aqueous media" as $\mathrm{H}^{+}$but is more accurately represented by the formula $\mathrm{H}_{3} \mathrm{O}^{+}$, as the proton is too readily captured to be capable of independent existence in the presence of a base. This means that reactions such as those shown in eq 2 to 5 will not occur unless a second base is present. For example, a combination of eq 2 and 4 gives

$$
\begin{aligned}
& \mathrm{H}_{2} \mathrm{O}+\mathrm{NH}_{3} \rightleftharpoons \mathrm{NH}_{4}{ }^{+}+\mathrm{OH}^{-} \\
& \text {acid }_{1} \text { base }_{2} \text { acid }_{2} \text { base }_{1}
\end{aligned}
$$

of 3 and 6 :

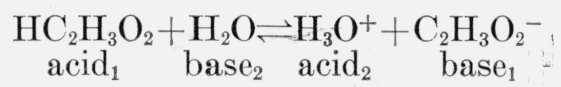

and 4 and 6 :

$$
\begin{aligned}
& \mathrm{H}_{2} \mathrm{O}+\mathrm{H}_{2} \mathrm{O} \rightleftharpoons \mathrm{H}_{3} \mathrm{O}^{+}+\mathrm{OH}^{-} \\
& \text {acid }_{1} \text { base }_{2} \text { acid }_{2} \text { base }_{1}
\end{aligned}
$$

In all cases, the net reaction is the transfer of a proton from one molecule or ion to another. $\mathrm{Acid}_{1}$ and base ${ }_{1}$ or acid and base $_{2}$ are termed "conjugate pairs."

It is obvious that any acid dissolved in water will react according to eq 8 , just as any derivative of ammonia will react according to eq 7 . The uniform behavior of all acids and of ammonia derivatives in dilute aqueous solution is thereby accounted for. Hantzsch, who studied various reactions of acids in different solvents, was the first person to recognize this clearly and he coined the expression "leveling effect" to describe the influence of water in masking differences in the intrinsic strength of acids $[6,7]$. Any solvent of basic character, such as liquid ammonia or pyridine, produces a comparable effect, giving $\mathrm{NH}_{4}{ }^{+}$, $\mathrm{C}_{5} \mathrm{H}_{5} \mathrm{NH}^{+}$, or in general solvated hydrogen ions. In a similar way, a solvent of acidic character, as for example glacial acetic acid, exerts a leveling effect upon bases [8 to 10].

It becomes evident that solvents can be divided into several categories. They are usually classified in the following way:

1. Amphiprotic solvents.-These solvents both give and take up protons with ease. Examples: Water, alcohols. Such solvents can undergo selfionization, as shown in eq 9. This reaction, termed "autoprotolysis," makes possible the construction of a $\mathrm{pH}$ scale for aqueous solutions of acids, bases, and salts.

2. Aprotic or inert solvents.-These solvents neither add nor release protons to an appreciable degree. Examples: Benzene and other hydrocarbons; chloroform and other halogenated hydrocarbons. Autoprotolysis and leveling effects do not occur. In these solvents perchloric acid, the strongest acid known, exhibits some tendency to dissociate into its ions, but most acids show practically no ionization. In aprotic solvents, most organic acids exist to some extent as double molecules or "dimers." Bases such as pyridine obviously cannot form ions. It is clear that the term $\mathrm{pH}$, which it will be recalled equals the negative logarithm of the hydrogen-ion activity, is not applicable to solutions of acids or bases in aprotic solvents. However, any pair of acids and bases will react with each other to an extent that depends upon their intrinsic acidic and basic strengths. Reactions of this kind can be followed with the aid of indicator dyes. As is generally known, an indicator dye is either an acid or a base, which in becoming a salt, undergoes a structural modification that is accompanied by a change of color. In some cases, the reaction of an acid and a base can be followed conductimetrically or potentiometrically, as most salts are somewhat ionized even in an inert solvent. These are the methods of greatest practical value, although other chemical methods are known.

3. Basic solvents.-Examples are liquid ammo- 
nia, aliphatic amines, aniline, pyridine, ethyl ether, dioxane, and esters. Basic solvents exert a pronounced leveling effect upon acids but not upon bases. In some cases, basic solvents exhibit weakly acidic properties and undergo slight autoprotolysis, as for example [11]:

$$
2 \mathrm{NH}_{3} \rightleftharpoons \mathrm{NH}_{4}++\mathrm{NH}_{2}^{-} .
$$

4. Acidic solvents.-Examples are concentrated sulfuric acid, glacial acetic acid, and other organic acids. These solvents exert a strong leveling effect on bases. Acidic solvents may be weakly basic, and autoprotolysis may occur to a very minor extent, as shown for acetic acid:

$$
2 \mathrm{CH}_{3} \mathrm{COOH} \rightleftharpoons \mathrm{CH}_{3} \mathrm{COOH}_{2}{ }^{+}+\mathrm{CH}_{3} \mathrm{COO}^{-} \text {. }
$$

In fact, Hantzsch attributed the electrolytic dissociation of perchloric acid in an aprotic solvent to a similar reaction, rather than to a dissociation into $\mathrm{H}^{+}$and $\mathrm{ClO}_{4}^{-}$ions.

The theory of the hydrogen bridge. - A second theory that is vital for the understanding of acidbase reactions is that of the "hydrogen bond," preferably termed the "hydrogen bridge" [12 to 23]. ${ }^{2} \quad$ This theory postulates that the proton may be shared between two atoms, X and Y, either of the same molecule or of different molecules, to form a bridge between $\mathrm{X}$ and $\mathrm{Y}$. In order to be capable of forming a hydrogen bridge, the proton must be part of an acidic group, which means that it is ordinarily attached to a halogen, oxygen, or nitrogen atom. In certain cases, it may be attached to a carbon atom holding a halogen or an unsaturated group, as in chloroform, hydrogen cyanide, or phenylacetylene. Atoms $\mathrm{X}$ and $\mathrm{Y}$ may be the same or different. For example, there are believed to be $\mathrm{OHO}$ bridges connecting the individual molecules of water and the alcohols, an FHF bridge in the anion of hydrogen fluoride, and NHN bridges connecting separate molecules of liquid ammonia. The weakly basic character of an aqueous solution of ammonium hydroxide is attributed to NHO bridges, and the dimerization of organic acids is attributed to $\mathrm{OHO}$ bridges. When $\mathrm{X}$ and $\mathrm{Y}$ are in the same molecule, as in salicylaldehyde, a so-called "chelate ring" is formed. In such cases, the hydrogen bridge may have a high degree of stability.

\footnotetext{
2 There is a voluminous literature on the hydrogen bridge The publications cited were selected mainly because of their general character and because they give references to many other articles on the subject.
}

Although there can be little question of its widespread occurrence, the nature of the hydrogen bridge is not clearly understood. The proton cannot share two pairs of electrons at one time, but it is possible that a proton originally attached to atom $\mathrm{X}$ penetrates to some extent an electron shell of atom Y. The usual explanation is that electronic resonance is set up, giving something intermediate between two extreme forms, such as $-\mathrm{O}-\mathrm{H}: \mathrm{NH}_{3}$ and $-\mathrm{O}^{-} \mathrm{H}:{ }^{+} \mathrm{NH}_{3}$. The simplest way of representing a hydrogen bridge can be illustrated by ammonium hydroxide: $\mathrm{NH}_{3}-\mathrm{H} \ldots \mathrm{OH}$. The linkage believed to be the weaker is indicated by a dotted line.

The concept of the hydrogen bridge necessitates a modification of Brönsted's hypothesis. He maintained that, as a consequence of his definition scheme eq 1, a conjugate pair of acids and bases do not possess the same electrical charge, so both cannot be electrically neutral molecules and at least one of them must be an ion. However, as we have just seen, a proton may be only partially surrendered by an acid and only partially captured by the base

The formation of the hydrogen bridge may therefore be regarded as incipient ionization, that is, an intermediate step in the complete transfer of a proton. The bond between hydrogen and atom $\mathrm{X}$ or $\mathrm{Y}$ is much weaker than an ordinary ionic or covalent bond, and rupture of a hydrogen bridge may lead to formation of either ions or neutral molecules. A familiar example is ammonia water, in which the following equilibria may be postulated:

$$
\mathrm{NH}_{3}+\mathrm{HOH} \rightleftharpoons \mathrm{NH}_{3} \mathrm{H} \ldots \mathrm{OH} \rightleftharpoons \mathrm{NH}_{4}{ }^{+}+\mathrm{OH}^{-} \text {(12) }
$$

The Lewis theory of acidity and basicity.-G. N. Lewis proposed a much broader concept of acids than that of Brönsted and Lowry [24 to 26]. Lewis stated that acidity and basicity have nothing to do with the presence of any specific element or group of elements, and that no particular substance is to be regarded as an acid or a base in an absolute sense. A base is defined as an electron donor and an acid as an electron acceptor. Neutralization is the formation of a coordinate covalent bond between the acid and the base. The adjective "coordinate" is inserted merely to indicate that both electrons are supplied by one of the interacting atoms, as the resulting bond is not different from other covalent bonds. The reaction occurs because a molecule of more stable electronic 
configuration results. An excellent presentation of the Lewis electronic theory and a discussion of its manifold applications, as well as a review of other acid-base theories, has been published recently [27]. ${ }^{3}$ The authors of this textbook draw attention to the analogy between the Lewis concept of acid-base reactions and the modern picture of oxidation-reduction reactions. Acids and oxidizing agents are electron acceptors, while bases and reducing agents are electron donors. However, there is a fundamental difference in the two types of reaction. In oxidation-reduction, electrons are transferred from the reducing agent to the oxidizing agent, whereas when an acid reacts with a base it gains only a share in a pair of electrons.

Lewis gave credit to Brönsted and Lowry for overcoming the reluctance of chemists to think of basicity in terms other than the hydroxyl ion. The list of bases is identical for both theories. However, while the Brönsted-Lowry theory limits acidic properties to proton donors the Lewis theory recognizes any electron acceptor as an acid. In addition to the hydrogen acids, the following compounds are included among Lewis acids: $\mathrm{BCl}_{3}, \mathrm{AlBr}_{3}, \mathrm{SnCl}_{4}, \mathrm{SO}_{3}, \mathrm{Ag}^{+}$, and $\mathrm{Cu}^{++}$. The reaction of ammonia with boron trichloride is an example of neutralization involving one of these acids:

$$
\mathrm{H}_{3} \mathrm{~N}:+\mathrm{BCl}_{3} \rightleftharpoons \mathrm{H}_{3} \mathrm{~N}: \mathrm{BCl}_{3} \text {. }
$$

The neutralization product is not necessarily a salt, but may be a covalent compound, as in the example cited. Lewis contended that "to restrict the group of acids to those substances which contain hydrogen interferes as seriously with the systematic understanding of chemistry as would the restriction of the term oxidizing agent to substances containing oxygen" [25]. However, he suggested that the term "hydrogen acids" or, for short, "H-acids" might be used to designate those acids that are proton donors. The great usefulness of Lewis's electronic theory of acids and bases in unifying a vast field of chemical reactions is clearly demonstrated in the references cited. Nonetheless, there are practical advantages as well as theoretical justification for considering the hydrogen acids as a special class. Kolthoff [30] has suggested that the Brönsted-Lowry defini-

\footnotetext{
${ }^{3}$ A more detailed review of the historical development of concepts of acids, bases, and salts can be found in a monograph by Walden [28] and in a series of articles published by the Journal of Chemical Education [29].
}

tion of an acid remain unchanged and that the larger group of acids recognized by Lewis be designated as "Lewis acids" or "proto-acids". To the writers, it is simpler and more logical to consider the Brönsted-Lowry acids as a special subclass, to be designated as hydrogen acids or H-acids, as suggested by Lewis. The acids with which the present investigations are concerned belong to this group, and the bases belong to the class of ammonia derivatives.

Object of the investigation.-Owing to the importance of water as a solvent, the behavior of acids and bases has been investigated very extensively in this medium, but relatively few systematic investigations in organic media have been conducted up to the present time. The result has been a laborious development of empirical and often unsatisfactory procedures, as well as a tendency to extrapolate concepts such as $\mathrm{pH}$ to systems in which this term is meaningless. Although academic studies have thrown considerable light upon the subject, there is as yet no systematic compilation of the knowledge so far obtained, and there is a great need for further investigation of acid-base phenomena in various solvents. The purpose of the present work is threefold: To aid in publicizing newer theories that are of considerable value in interpreting and predicting the behavior of acids and bases in organic solvents, as well as the experimental data upon which they are founded; to obtain systematic data in regard to acid-base reactions in organic media, particularly in those solvents that are very dissimilar to water, such as the aprotic solvents; and to assist in the development of practical methods for measurement of acidity and basicity in organic solvents.

The study of acid-base reactions in any medium may be divided into two phases:

1. Determination of the relative strengths of acids and bases. - In aqueous solutions, these are indicated by the $\mathrm{pH}$ values for equimolar concentrations of acids or bases. The $\mathrm{pH}$ value for an acid, HX, is a measure of the extent to which the base $\mathrm{X}^{-}$, present in relatively low concentration, can compete for the proton with the base water, which is very weak but present in high concentration. As stated above, the term pH is not applicable to aprotic solvents. However, it is possible to ascertain the relative strengths of a series of acids in an aprotic solvent in terms of the extent of their reactions with some reference base, such 
as a basic indicator dye. Similarly, the relative strengths of organic bases in an aprotic medium can be determined by measurement of their reactivity with an acidic indicator dye. An alternative method is to measure the extent to which an acid (base) competes with an indicator acid (base) for a reference base (acid).

2. Determination of total acidity or basicity.This is accomplished by titration with a strong base or acid. The indicator method for detecting the endpoint is usually the simplest and most convenient, provided indicators of satisfactory properties are available. An indicator should not only undergo color changes in the spectral range to which the eye is sensitive but should be readily soluble and stable towards light and chemical reagents other than acids or bases. Moreover, because colored impurities or suspended particles often render one indicator useless, it is desirable to have a substitute with the same acidic or basic strength but with a different color change. Considerable effort has been expended by a number of investigators in the development of indicators for aqueous media, but only a few of these indicators are suitable for organic media. Before acid-base measurements of organic solutions can be performed as readily as those of aqueous solutions, it will be necessary to provide a series of indicators, ranging from strongly acidic to strongly basic compounds. A further need is for stable, crystalline organic acids and bases sufficiently strong to neutralize weak bases and acids. Work along these lines by the writers is in progress, and it is hoped that others will direct their efforts toward the same objective.

The investigations reported in this paper included the synthesis of the indicator, bromphthalein magenta, and a study of its reactions with different types of organic bases in benzene and other organic solvents. Interesting and unexpected color phenomena were observed, which at first were very puzzling. A consistent explanation has been found that is in harmony with the theories just reviewed, as well as with current explanations for the relation of color to chemical constitution, and is applicable to other indicators. It is believed that these studies point to useful applications of the indicator and that they contribute to an understanding of acid-base phenomena in organic media.

\section{Apparatus and Method}

The absorption spectra were obtained with a Beckman quartz photoelectric spectrophotometer [31], in which the original cell compartment had been replaced by one designed to hold a pair of 3.8-cm (inside diameter) cylindrical cell holders. A photograph of the parts of the cell holder is shown in figure 1, A. In part of the experiments,

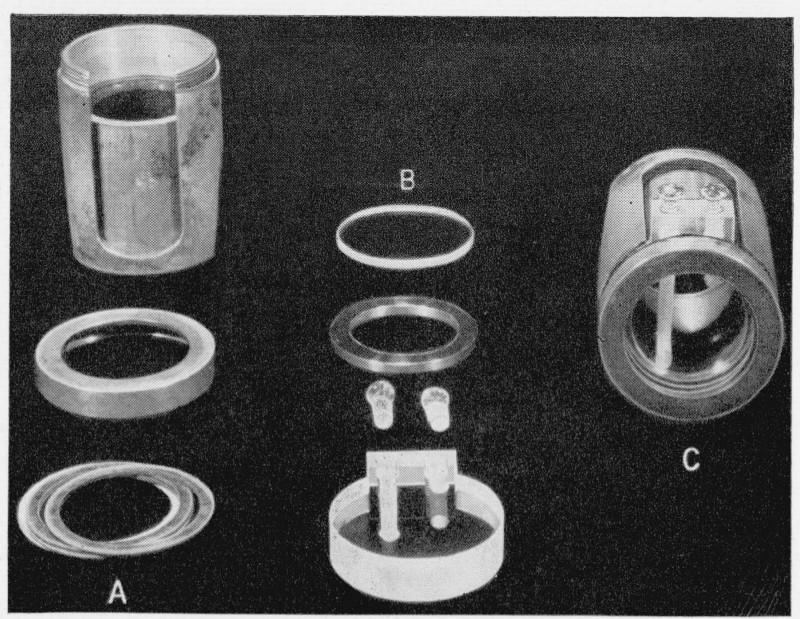

FiguRE 1. Absorption cell used in spectrophotometric measurements, and its component parts.

From top to bottom: $A$, Cell holder with metal spacer, threaded cover, gaskets; $B$, quartz endplate, stainless steel spacer, tubulated endplate and stoppers; $C$, cell assembled in the holder.

each cell consisted of a stoppered quartz cylinder $3.8 \mathrm{~cm}$ in diameter and $1 \mathrm{~cm}$ in length, open and polished at the ends, and fitted with a pair of matched crystalline quartz endplates that were $2 \mathrm{~mm}$ in thickness. The cylinder and endplates were assembled tightly in the cell holder by means of a combination of metal spacers and Bakelite and rubber gaskets. To provide a cell $5 \mathrm{~mm}$ in length, the quartz cylinder was replaced by a ring of monel metal, gold-plated. This ring (3.8 $\mathrm{cm} \mathrm{o.} \mathrm{d.} \mathrm{and} 2.8 \mathrm{~cm} \mathrm{i.} \mathrm{d.)} \mathrm{was} 5 \mathrm{~mm}$ in thickness. Two tubular openings through the rim, fitted with glass stoppers, were provided for filling the assembled cell and removing air bubbles.

As a convenient means for obtaining still shorter cells of variable lengths, a new type of tubulated endplate was designed (fig. 1, B, and fig. 2). This was cut in one piece from fused quartz to a thickness of $1 \mathrm{~cm}$, and consisted of a circular plate 3.8 $\mathrm{cm}$ in diameter having a neck $2 \mathrm{~cm}$ in width and 1 $\mathrm{cm}$ in length. Two tubular openings of unequal 


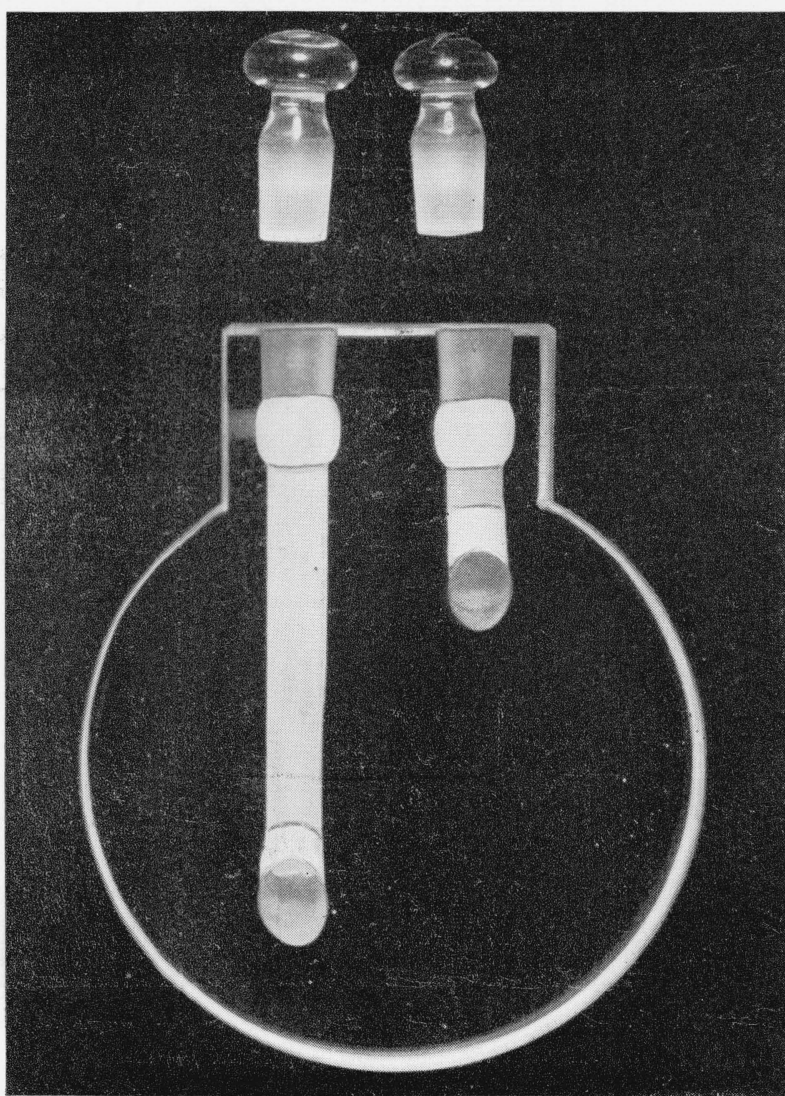

Figure 2. Tubulated quartz endplate with stoppers.

length were drilled perpendicularly through the neck and continued through the plate so as to emerge at an angle on one face of the plate. The spacing between the openings provided for noninterference with the light beam. Glass stoppers were ground to fit the openings in the neck. When assembled in the cell holder with a stainless steel spacer of the desired thickness and a 2-mm thick crystalline quartz endplate (fig. 1, C), this provided a very satisfactory absorption cell for measurements in the visible spectrum. Two such cells that contained either water or benzene were well matched between 390 and $700 \mathrm{~m} \mu$, but did not show equal absorption in the ultraviolet region. When compared with a cell having two crystalline quartz endplates, they showed a very narrow absorption band near $240 \mathrm{~m} \mu$, which was probably due to a trace of iron in the fused quartz. The intensity of this band was different in the two tubulated endplates. Stainless steel spacers of thicknesses $1,2.5$, and $5 \mathrm{~mm}$ were made, to pro- vide absorption cells of these respective lengths. ${ }^{4}$ The solution was introduced into the cell through the longer tube.

In all the spectrophotometric measurements, a pair of cells was used in the customary way. Ordinarily the reference cell contained the pure solvent ${ }^{5}$ and its companion cell contained the solution under investigation. The solutions were prepared and the measurements were performed in a room kept at $25^{\circ} \mathrm{C}$. By passing a stream of air through the cell compartment, the increase in temperature caused by proximity of the light source was limited to the range $26^{\circ} \pm 1^{\circ} \mathrm{C}$. Solutions of known concentrations were prepared and diluted quantitatively. Volumetric flasks and pipettes calibrated at the National Bureau of Standards were used throughout. The microbalance used for the preparation of most solutions of dyes gave a precision of 0.5 to 1.0 percent for weights of the order used. Readings were made at intervals of 5 or $10 \mathrm{~m} \mu$. Measurements of the percent transmittancy were usually reproducible within \pm 0.2 percent.

In some of the figures to follow, the transmittancies in percent and wavelengths in millimicrons are plotted as ordinates and abscissas, respectively. The resulting curves are called "transmittancy curves." In other figures, values for the molar absorbancy index, $a_{M}$, are given as ordinates. These were calculated by means of the equation,

$$
a_{M}=\frac{-\log _{10} T_{s}}{b \times M},
$$

in which $: T_{s}=T_{\text {soln }} / T_{\text {solv }}=$ the transmittancy of the solute, $b$ is the depth of the cell in centimeters, and $M$ is the molar concentration of the solution. ${ }^{6}$ In the latter figures, both the wavelength in millimicrons and the wave number (reciprocal of the wavelength) in centimeters ${ }^{-1}$ are given as abscissas, at the top and bottom, respectively, of the figures. These curves are referred to as "molar absorbancy curves."

\footnotetext{
4 Since completion of the experimental work described in this paper, combination of a tubulated endplate with a thin quartz plate that has a central indented area 0.1 or $0.5 \mathrm{~mm}$ in depth has given satisfactory cells of still shorter length.

${ }^{5}$ Exceptions will be noted in the text.

6 The symbols and terminology used were recently adopted for use in NBS publications and are discussed in NBS Letter Circular LC857 (May 19, 1947). The term "molar absorbancy index" is used in preference to "molar absorption index" or "molar absorption coefficient."
} 


\section{Materials}

\section{Indicator Dyes}

(a) Preparation of the ethyl ester of tetrabromophenolphthalein ("bromphthalein magenta $\mathrm{E}^{\prime \prime 7}$ ).

Phenolphthalin was prepared from phenolphthalein by a procedure based upon Baeyer's brief description [32] and was then converted successively to phenolphthalin ethyl ester, tetrabromophenolphthalin ethyl ester, the potassium salt of tetrabromophenolphthalein ethyl ester, and tetrabromophenolphthalein ethyl ester. The entire series of transformations is shown in figure 3 .
15 minutes with $100 \mathrm{ml}$ of a 25 -percent solution of ammonium chloride. The ammonium chloride solution was then decanted, and the zinc was washed twice with distilled water. The activated zinc was added to a $500-\mathrm{ml}$ round-bottom flask that contained a solution of phenolphthalein (0.25 mole, Eastman No. 202) in $125 \mathrm{ml}$ of $10-M$ sodium hydroxide, and the mixture was heated to boiling while constantly stirred. Near the boiling point, the solution became colorless. Heating was continued on the steam bath for an hour. After the addition of $150 \mathrm{ml}$ of $6-N$ sodium hydroxide, the mixture was filtered through
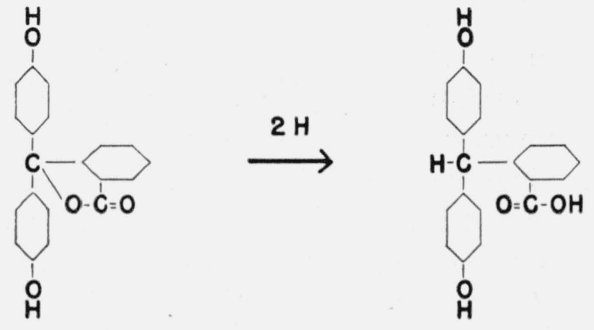

$\dot{I}$

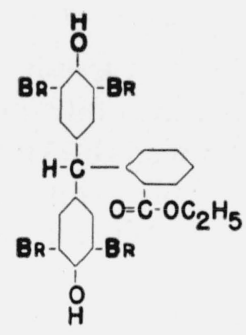

II

II
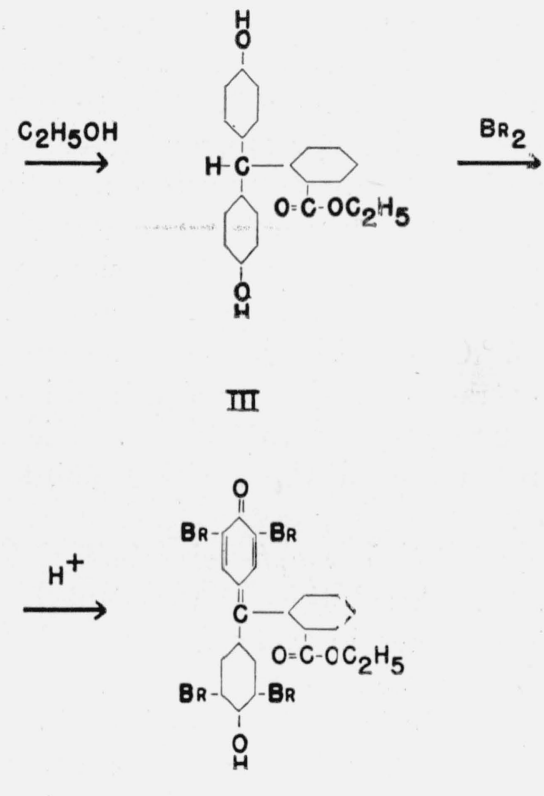

ZII

Figure 3. Outline of method for preparing bromphthalein magenta E.

I, Phenolphthalein; II, phenolphthalin; III, phenolphthalin ethyl ester; IV, tetrabromophenolphthalin ethyl ester; V, potassium salt of tetrabromophenolphthalein ethyl ester; VI, tetrabromophenolphthalein ethyl ester ("bromphthalein magenta E.")

The last four steps were performed essentially by the process outlined by Nietzki and Burckhardt [33]. However, experiments with several small batches were conducted to determine the most favorable conditions, and at various stages modifications were introduced. The following description applies to the procedures found to be most satisfactory.

Preparation of phenolphthalin (II, fig. 3).-Zinc dust (0.76 mole) was "activated" by stirring for

\footnotetext{
7 This name was coined as a substitute for the cumbersome chemical terminology and is intended to suggest the relation of the compound to phenolphthalein and to the sulfonephthalein indicators. The suggested abbreviation is BPM. The suffixes $\mathrm{E}$ and $\mathrm{B}$ denote the ethyl and $n$-butyl esters, respectively.
}

asbestos to remove the excess of zinc. The residue of zinc was heated to boiling with $6-N$ sodium hydroxide, and the mixture again filtered through asbestos. The combined filtrates were then poured into a slight excess of $3.6-N$ hydrochloric acid $(500 \mathrm{ml})$. The flocculent precipitate was digested with water on the steam bath and then washed several times by decantation. Finally, it was filtered with the aid of suction and dried in a vacuum oven at $100^{\circ} \mathrm{C}$. The yield was 77 percent. The product $\left(\mathrm{mp}, 232^{\circ} \text { to } 236^{\circ} \mathrm{C}\right)^{8}$ may be used without further purification or recrystallized

\footnotetext{
${ }^{8}$ All melting points were determined with an ASTM thermometer for 3-in. immersion, calibrated at the National Bureau of Standards.
} 
from $n$-butyl alcohol (about $50 \mathrm{ml}$ of hot butyl alcohol to $10 \mathrm{~g}$ of phenolphthalin). The recrystallized product melted at $235^{\circ}$ to $235.5^{\circ} \mathrm{C}$. Baeyer reported the melting point of phenolphthalin to be $225^{\circ} \mathrm{C}$, but in catalog No. 35 of Eastman Organic Chemicals it is given as $232^{\circ}$ to $236^{\circ} \mathrm{C}$.

Preparation of phenolphthalin ethyl ester (III, fig. 3).-Phenolphthalin (0.2 mole) was dissolved in 1 liter of absolute ethyl alcohol, the solution saturated with dry hydrogen chloride, and the flask closed tightly with a glass stopper. After the solution had stood for several days at room temperature, it was concentrated to $400 \mathrm{ml}$ on the steam bath and then poured onto ice, whereupon a curdy solid precipitated. The yield of air-dried product was 100 percent. The solid was dissolved in $100 \mathrm{ml}$ of ether and extracted with small portions of a 10-percent solution of sodium carbonate until the extract was no longer pink. The ether was evaporated and the residue was dissolved in 1.5 liters of hot benzene. Flat needles $\left(\mathrm{mp}, 156^{\circ}\right.$ to $157^{\circ} \mathrm{C}$ ) were obtained in 82.5-percent yield. After extraction with carbon tetrachloride the residue melted at $157^{\circ}$ to $158^{\circ} \mathrm{C}$. Nietzki and Burckhardt reported the melting point as $156^{\circ}$ to $158^{\circ} \mathrm{C}$.

Preparation of tetrabromophenolphthalin ethyl ester (IV, fig. 3).-A solution of phenolphthalin ethyl ester (0.15 mole) in $225 \mathrm{ml}$ of 95 -percent ethyl alcohol was cooled to $0^{\circ} \mathrm{C}$, and was then shaken constantly during the dropwise addition of bromine $(0.6$ mole). The temperature of the mixture was not allowed to rise above $15^{\circ} \mathrm{C}$. The mixture was placed in a refrigerator for half a day, during which time a large crop of granular yellowish crystals formed. The precipitate was filtered with the aid of suction, and the yellow color was removed by washing with small portions of alcohol. The yield of air-dried product (mp, $161^{\circ}$ to $162^{\circ} \mathrm{C}$ ) was 73 percent. A sample recrystallized from 95-percent alcohol containing Darco charcoal gave irregular prismatic crystals that melted at $162.5^{\circ}$ to $163.5^{\circ} \mathrm{C}$. Additional dark yellow, sticky solid was obtained by dilution of the alcoholic filtrate and washings with about 2 liters of water. Crystallizing this from alcohol produced a solid tinged with light yellow and brought the total yield to 84 percent. Nietzki and Burckhardt performed the bromination in glacial acetic acid and obtained a product that melted at $163^{\circ}$ after recrystallization from acetic acid. No yield was reported. Glacial acetic acid and carbon tetrachloride were found by us to be less satisfactory solvents than ethyl alcohol, from which the product separated more readily and in a purer condition.

Preparation of the potassium salt of tetrabromophenolphthalein ethyl ester (V, fig. 3).-Tetrabromophenolphthalin ethyl ester $(0.1$ mole $)$ was dissolved in a cold solution of 0.4 mole of potassium hydroxide in $200 \mathrm{ml}$ of water, yielding a colorless solution. The solution was mechanically stirred while potassium ferricyanide $(0.2$ mole in $200 \mathrm{ml}$ of water) was added rapidly. After about 5 minutes, the dark blue slimy precipitate was filtered with suction, and the moist product was dried on a clay plate. ${ }^{9}$ The air-dried solid, in small portions, was extracted with 95-percent ethyl alcohol in a Soxhlet apparatus. A dull green, crystalline solid separated from the alcoholic extracts (total yield, 55 percent). A white residue in the thimbles was identified as potassium ferrocyanide. A second extraction of the solid with 95-percent alcohol yielded lustrous green crystals.

Preparation of tetrabromophenolphthalein ethyl ester, "bromphthalein magenta E" (VI, fig. 3).The potassium salt of tetrabromophenolphthalein ethyl ester was converted into tetrabromophenolphthalein ethyl ester in portions of $5 \mathrm{~g}$, under slight variations of the following procedure. The potassium salt was suspended in $100 \mathrm{ml}$ of water, and acetic acid was added in small portions until, after thorough shaking, the color of the solid had changed to brick red. Benzene $(200 \mathrm{ml})$ was then added and the mixture was heated on the steam bath under reflux. After half an hour, during which the mixture was occasionally shaken, all but a trace of the brick-red solid had dissolved in the benzene. The orange-red benzene layer was separated, partly dried by filtration through filter paper and, while still hot, was divided between two 250-ml Erlenmeyer flasks and placed in a desiccator that contained calcium chloride and vaseline. The desiccator was connected with the water pump. The solution became slightly turbid but cleared after boiling started. As soon as the liquid became concentrated and cooled by the evaporation of solvent, crystals began to form. These consisted of short, silky, light-yellow needles, which at first aggregated in a sheaf-like arrange-

${ }^{8}$ Subsequent batches were centrifuged at this point with considerable saving of time. 
ment then changed into fluffy balls that finally filled the liquid. During filtration these crystals lost a considerable part of their bulk and changed to a bright orange powder. On heating for a few hours in a vacuum oven at $85^{\circ}$ to $105^{\circ} \mathrm{C}$, the color changed to brick-red. The total yield of oven-dried product from $41.5 \mathrm{~g}$ of the potassium salt was 85 percent. The products from five separate preparations gave identical absorption spectra when dissolved in benzene. Recrystallization of $31 \mathrm{~g}$ from 1.5 liters of benzene gave 91 percent of oven-dried product that had an unchanged absorption spectrum.

Several experiments were performed to determine whether the benzene of crystallization in the air-dried solid was present in stoichiometric proportions. The results were inconclusive, but may indicate the presence of 1 molecule of benzene of crystallization, which is slowly lost at atmospheric conditions. When heated at atmospheric pressure, the orange powder dries to a porous mass that can be ground to a brick-red powder. The solid melts to a deep-red liquid at about $209^{\circ}$ to $210^{\circ}$ C. Nietzki and Burckhardt obtained stout blood-red prisms (mp, about $210^{\circ}$ to $215^{\circ} \mathrm{C}$ ) from alcohol.

Analysis: ${ }^{10}$ Calculated for $\mathrm{C}_{22} \mathrm{H}_{14} \mathrm{O}_{4} \mathrm{Br}_{4}$ : C, 39.9; $\mathrm{H}, 2.1 ; \mathrm{Br}, 48.3$. Found: $\mathrm{C}, 40.5 ; \mathrm{H}, 2.3 ; \mathrm{Br}$, 48.2. For the product of Nietzki and Burckhardt the following analysis was reported: C, 39.4; $\mathrm{H}, 2.3 ; \mathrm{Br}, 49.4$.

(b) Preparation of the $n$-butyl ester of tetrabromophenolphthalein ("bromphthalein magenta B")

Preparation of phenolphthalin $n$-butyl ester.Phenolphthalin (0.1 mole) was dissolved in 150 $\mathrm{ml}$ of hot $n$-butyl alcohol (commercial high grade). The solution was saturated with dry hydrogen chloride and heated on the steam bath for several days. The excess of butyl alcohol was distilled off under reduced pressure, and the residue in the distilling flask was poured onto ice. The thick sirup that formed was converted into a granular solid after continued stirring. The suction-dried precipitate was dissolved in ether, and the ether solution was extracted with 10-percent sodium carbonate solution until the extract showed only a faint pink color. The ether layer was then washed with water and finally evaporated to dryness on the steam bath. When the residue was

${ }_{10}$ All the analyses were performed by Kenneth D. Fleischer. dissolved in $100 \mathrm{ml}$ of hot benzene, a yellow solution was formed from which small needles precipitated on cooling. The needles were filtered and washed with cold benzene until no color remained. After 4 hours in a vacuum oven at $80^{\circ} \mathrm{C}, 78$ percent of product $\left(\mathrm{mp}, 131^{\circ}\right.$ to $\left.131.5^{\circ} \mathrm{C}\right)$ was obtained.

Analysis: Calculated for $\mathrm{C}_{24} \mathrm{H}_{24} \mathrm{O}_{4}$ : C, $76.6 ; \mathrm{H}$, 6.4. Found: C, 76.7; H, 6.7.

Preparation of tetrabromophenolphthalin-n-butyl ester.-The same procedure was followed as for the preparation of the corresponding ethyl ester, except that $n$-butyl alcohol was used in place of ethyl alcohol as the solvent for bromination. Phenolphthalin $n$-butyl ester (0.2 mole) gave a 78 percent yield of the tetrabromo-derivative. The melting point $\left(138^{\circ}\right.$ to $\left.138.4^{\circ} \mathrm{C}\right)$ was raised to $139.5^{\circ}$ to $139.8^{\circ} \mathrm{C}$ by recrystallization from methyl alcohol.

Preparation of the potassium salt of tetrabromophenolphthalein n-butyl ester.-The procedure corresponded to that used for the ethyl ester. The largest quantity used in an experiment was 0.03 mole of tetrabromophenolphthalin $n$-butyl ester. Acetone was used for extraction of the product in a Soxhlet apparatus. Lustrous green crystals were obtained.

Preparation of tetrabromophenolphthale in $n$-butyl ester, "bromphthalein magenta B."-The method was similar to that used for the preparation of bromphthalein magenta $\mathrm{E}$. The oven-dried product (yield from 0.0275 mole, 72 percent) was bright orange in color and melted and partly decomposed at about $110^{\circ} \mathrm{C}$.

Analysis: Calculated for $\mathrm{C}_{24} \mathrm{H}_{18} \mathrm{O}_{4} \mathrm{Br}_{4}$ : C, 41.8; $\mathrm{H}, 2.6 ; \mathrm{Br}, 46.3$. Found: C, $42.2 ; \mathrm{H}, 3.0 ; \mathrm{Br}, 47.4$. The absorption spectrum of a $5 \times 10^{-5}-M$ solution of bromphthalein magenta $\mathrm{B}$ in benzene did not differ significantly from that of an equimolar solution of bromphthalein magenta $\mathrm{E}$. The synthesis of bromphthalein magenta B has not hitherto been reported.

\section{Organic Bases}

The best commercial grade of organic bases was used in all cases. Samples of bases that are available only in research quantities were obtained through the courtesy of several manufacturers. A list of the bases, which includes 14 primary aliphatic amines, 18 secondary aliphatic amines, 12 tertiary aliphatic amines, and 6 guanidine deri- 
vatives, is given in table 1 . The source and the method of purification, if any, are indicated in the footnotes at the end of the table. The preparation of tetraalkylammonium salts is described later in the text. Reference will also be made to trimethylamine oxide, certain alkaloids, and guanidine salts.

TABLE 1.-Source of organic bases and method of purification

\begin{tabular}{|c|c|c|}
\hline $\begin{array}{l}\text { Primary aliphatic } \\
\text { amines }\end{array}$ & $\begin{array}{c}\text { Secondary aliphatic } \\
\text { amines }\end{array}$ & $\begin{array}{c}\text { Tertiary aliphatic } \\
\text { amines }\end{array}$ \\
\hline 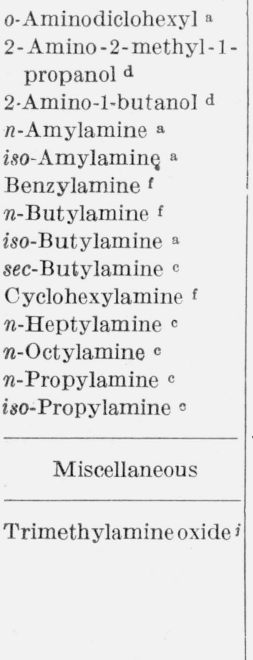 & 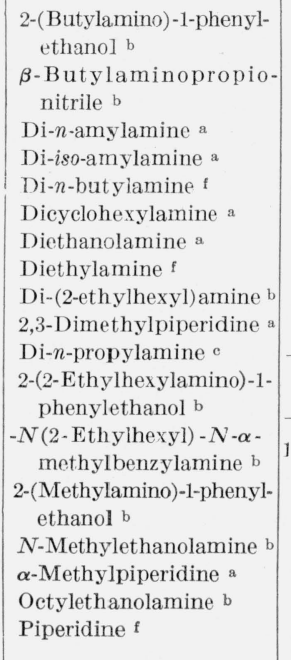 & 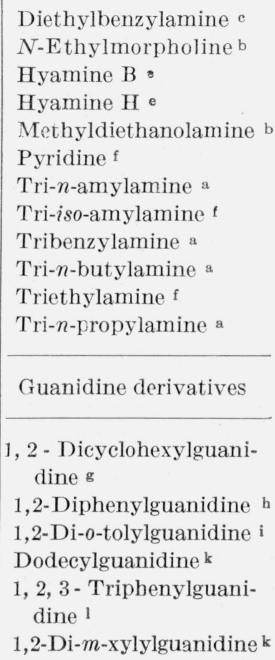 \\
\hline
\end{tabular}

a Eastman Kodak Co.; no further treatment.

b Carbide \& Carbon Chemicals Corp.; no further treatment.

c Sharples Chemicals Inc.; no further treatment.

d Commercial Solvents Corp.; no further treatment.

e Röhm and Haas; no further treatment.

\& Eastman Kodak Co.; dried over sodium hydroxide and redistilled.

g American Cyanamid Co.; isolated from hydrochloride, recrystallized from toluene, and dried in a vacuum oven at $100^{\circ} \mathrm{C}, \mathrm{Mp}, 180^{\circ}$ to $181^{\circ} \mathrm{C}$.

h Eastman Kodak Co.; recrystallized twice from toluene and dried in a vacuum oven at $75^{\circ} \mathrm{C} ; \mathrm{Mp}, 147.5^{\circ}$ to $147.8^{\circ} \mathrm{C}$.

i Eastman Kodak Co.; recrystallized twice from benzene and dried in a vacuum oven at $100^{\circ}$ to $110^{\circ} \mathrm{C} ; \mathrm{Mp}, 175^{\circ}$ to $175.5^{\circ} \mathrm{C}$.

j Synthesized from trimethylamine; $\mathrm{mp}, 94.5^{\circ}$ to $95.5^{\circ} \mathrm{C}$ (dihydrate).

* American Cyanamid Co.; no further treatment.

1 Eastman Kodak Co.; recrystallized once from cyclohexane and dried in air; $\mathrm{mp}, 144.5^{\circ}$ to $145.5^{\circ} \mathrm{C}$.

\section{Solvents}

Benzene.-A high grade of commercial solvent was dried over Drierite and redistilled [34].

Cyclohexane.-A high-grade solvent suppled for spectroscopic work was used without further treatment.

Dioxane.-Commercial solvent was purified according to the method of Eigenberger [35].

Ethyl acetate.-A high grade of commercial sol- vent was shaken with dilute alkali, washed, dried, and distilled [34].

Ethyl alcohol.-95-percent alcohol was redistilled [34].

Ethylene dichloride (1, 2-dichloroethane).-A high grade of commercial solvent was used. This showed no acidity when freshly opened. If stored in a colorless glass container in indirect daylight, the solvent acquired an acidic reaction in a few weeks. Deterioration was very slow when the solvent was protected from light.

Other solvents used in qualitative tests were high-grade commercial specimens.

\section{Data and Discussion}

\section{History of Bromphthalein Magenta}

The dye referred to as "bromphthalein magenta E" was first synthesized in 1897 [33] in the course of studies of the structures of phenolphthalein and related compounds. In a similar connection, it has been the subject of spectrographic studies by Thiel and Diehl [36], and by Madame RamartLucas and her collaborators [37, 38]. The dye and its potassium salt have been proposed for the detection of native protein [39].

Bromphenol blue, the sulfonephthalein analog of bromphthalein magenta, has been used as an indicator in acid-base studies in hydrocarbons and their halogenated derivatives, such as benzene [40 to 43] and chlorobenzene [44], as well as in acetonitrile [45]. It has limited solubility in benzene (about $10^{-4}$ mole per liter) and tends to form precipitates with certain amines. Furthermore, two changes in color, namely from colorless to yellow and from yellow to blue, complicate its use as an indicator [43]. It had been thought likely that the ethyl ester of tetrabromophenolphthalein ("bromphthalein magenta E," "BPM-E") would show a higher solubility in hydrocarbons and related solvents, as well as a single color change from yellow to blue or purple upon reaction with an organic base. The first expectation was fulfilled, ${ }^{11}$ and the products of its reaction with secondary aliphatic amines or tetraalkylammonium hydroxides give blue solutions in benzene. However, the interesting discovery was made that tertiary aliphatic amines and symmetrical di- or triaryl-substituted guanidines react with bromphthalein magenta to give magenta solutions in

\footnotetext{
11 The solubility in benzene is more than $10^{-3}$ mole per liter.
} 
benzene and like solvents. Primary aliphatic amines display hybrid behavior, giving red-violet solutions whose absorption spectra show resemblances to those of both secondary and tertiary amines. Furthermore, there are characteristic differences in the blue color and absorption spectra of tetraalkylammonium derivatives of bromphthalein magenta, on the one hand, and solutions of the dye to which secondary amines have been added. These and other color phenomena are discussed in following sections. At this point, however, it may be noted that the differentiating behavior of bromphthalein magenta towards various classes of bases in benzene is not unique but is shown by bromphenol blue and other sulfonephthaleins as well as by dyes of several other classes [43].

Replacement of the ethyl radical in the carbethoxy group by a $n$-butyl radical would not be expected to affect the behavior of bromphthalein magenta as an indicator in a noticeable way but might confer increased solubility in hydrocarbons. Bromphthalein magenta $\mathrm{B}$ is indistinguishable from bromphthalein magenta $\mathrm{E}$ as an indicator, although it is five or more times as soluble in benzene on a molar basis. In cyclohexane, a $10^{-4}-M$ solution of bromphthalein magenta $\mathrm{B}$ could be prepared, but the solubility of bromphthalein magenta $\mathrm{E}$ was too small for use in this solvent.
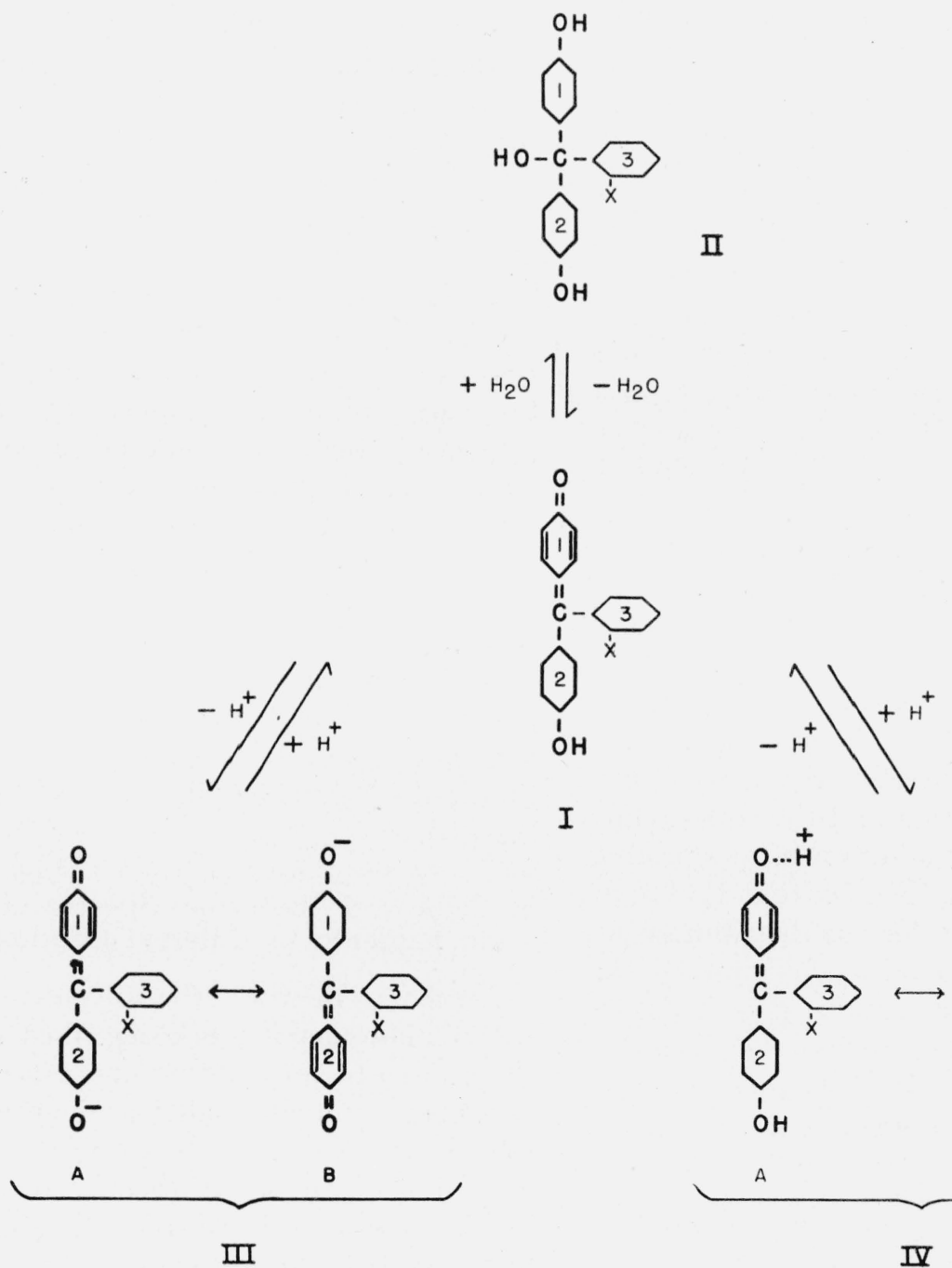

\section{I}

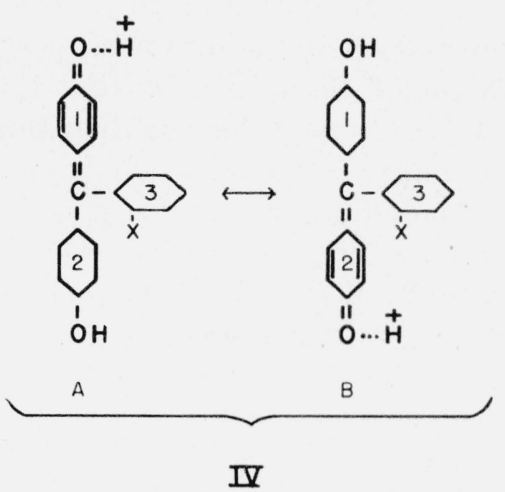

Figure 4. Structural and color changes of benzaurin.

I, Benzaurin ( $\mathrm{X}=\mathrm{H})$ (yellow); II, carbinol (colorless); III, anion (deep color); IV, cation (deep color). 


\section{Structure of Bromphthalein Magenta}

Bromphthalein magenta ( $\mathrm{E}$ or $\mathrm{B})$ belongs to the broad class of triphenylmethane dyes. More specifically, it may be regarded as a derivative of the simplest benzein dye, benzaurin. ${ }^{12}$ The formula of benzaurin (I) is given in figure 4. The position marked $\mathrm{X}$ in ring 3 is that occupied by $-\mathrm{H}$ in benzaurin, by the group $-\mathrm{COOC}_{2} \mathrm{H}_{5}$ in bromphthalein magenta $\mathrm{E}$, and by the group $-\mathrm{COOC}_{4} \mathrm{H}_{9}$ in bromphthalein magenta $\mathrm{B}$. The latter two compounds have 4 bromine atoms in place of the hydrogen atoms ortho to the $\mathrm{C}-\mathrm{O}$ groups in rings 1 and 2. For simplification of the figure, the bromine atoms have been omitted. The sulfonephthalein dyes are likewise related to benzaurin, having $\mathrm{X}=-\mathrm{SO}_{3} \mathrm{H}$. Substituents in position $\mathrm{X}$ have often been found to protect triphenylmethane dyes from the addition of a molecule of water, which would give the colorless carbinol (II, fig. 4). It is likely, also, that they produce a slight modification of the color of the dyes. ${ }^{13}$

However, the groups that are most actively involved in the structural changes and the accompanying shifts in spectral absorption, which occur when a dye of the benzaurin type is transformed to its anion or cation, are the methane carbon atom and the quinoid (ring 1 ) and phenolic (ring 2 ) groups. The simplest explanation of the color phenomena involved is based upon the present-day concept of electronic resonance. ${ }^{14}$ The neutral molecule gives yellow solutions in hydrocarbons and halogenated hydrocarbons because the well known chromophoric or "color-bearing" quinoid group $\mathrm{O}=\mathrm{C}_{6} \mathrm{H}_{4}=$ is present in the molecule. The anion (III, fig. 4) is formed by the loss of one proton from the $-\mathrm{OH}$ group, and the cation (IV, fig. 4) is produced by the addition of one proton to $=\mathrm{O}$. In figure 4 , it can be seen that III, B, is the same as III, A, except for the distribution of electrons. A similar relationship holds for IV, A, and IV, B. The anion (or cation) is believed to have a hybrid structure, intermediate between the extremes $\mathrm{A}$ and $\mathrm{B}$, due to the resonance between these two forms. ${ }^{15}$ Such resonance is considered

\footnotetext{
12 Its relationship to phenolphthalein has already been noted.

13 This has been found to be true for basic triphenylmethane dyes. Dyes of the benzaurin class have been less thoroughly studied [38].

14 Equivalent to the British term "mesomerism."

15 The double-headed arrow is often used to indicate resonance among different structures [23]. A and B are the principal but not the sole contributors to the hybrid anion (or cation).
}

to cause a shift in the absorption of light, from the ultraviolet to the visible portion of the spectrum. When this occurs, the substance in question transmits light of one of the deeper colors, for example red, violet, blue, or green [17, 23, 36, 46 to 50].

The cation form of bromphthalein magenta (red in color) can be obtained by dissolving the dye in concentrated sulfuric acid. The dye in this case is acting as a base. However, its behavior as an acid is of principal interest. It is well known that phenol shows acidic properties that are enhanced by substitution of halogen or nitro groups in the ortho position. This principle was used to advantage in the synthesis of certain sulfonephthalein indicators [51]. An explanation for the acidic behavior of phenol based upon the resonance concept has been given [17, 23]. According to this view, the $-\mathrm{OH}$ group lies in the plane of the benzene ring, with the $-\mathrm{H}$ directed toward one of the ortho positions. Studies of the infrared spectra of phenol, o-bromophenol, 2,4,6tribromophenol, and other substituted phenols have led to the belief that, when ortho positions in phenol are substituted by halogen atoms, the proton of $-\mathrm{OH}$ is displaced to a small extent toward an adjacent halogen atom $[52,53]$. When the same ideas are applied to bromphthalein magenta, which has a structural relationship to tribromophenol, its acidic properties may be accounted for and it can be predicted that, because the proton of $-\mathrm{OH}$ is attracted toward a neighboring bromine atom within the molecule, the tendency for intermolecular association of the $-\mathrm{OH}$ groups to give dimers or higher polymers [16] will be absent. In other words, bromphthalein magenta should exist in monomeric form in solution and is therefore likely to conform to Beer's law [54].

\section{Color and Absorption Spectra of Bromphthal- ein Magenta in Different Organic Solvents}

Benzene.--Benzene, the solvent for most of the spectrophotometric work described in this paper, was chosen because of its importance as a solvent, availability in high quality at moderate cost, good solvent properties, aprotic character, stability, and miscibility with other organic solvents. In the range of concentrations studied, namely, $1.6 \times 10^{-5}$ to $3 \times 10^{-4} \mathrm{M}$, the absorption spectra of BPM-E and BPM-B coincided within experimental error and showed no deviations from Beer's law. The 
absorption spectrum of a $10^{-4}-M$ solution of BPM-E showed no change after 4 months, during which time it was protected only from evaporation and from direct sunlight.

The absorbancy curve obtained for $5 \times 10^{-5}-M$ bromphthalein magenta $\mathrm{E}$ in benzene is shown in figure 5 (curve 1). The high absorption of benzene

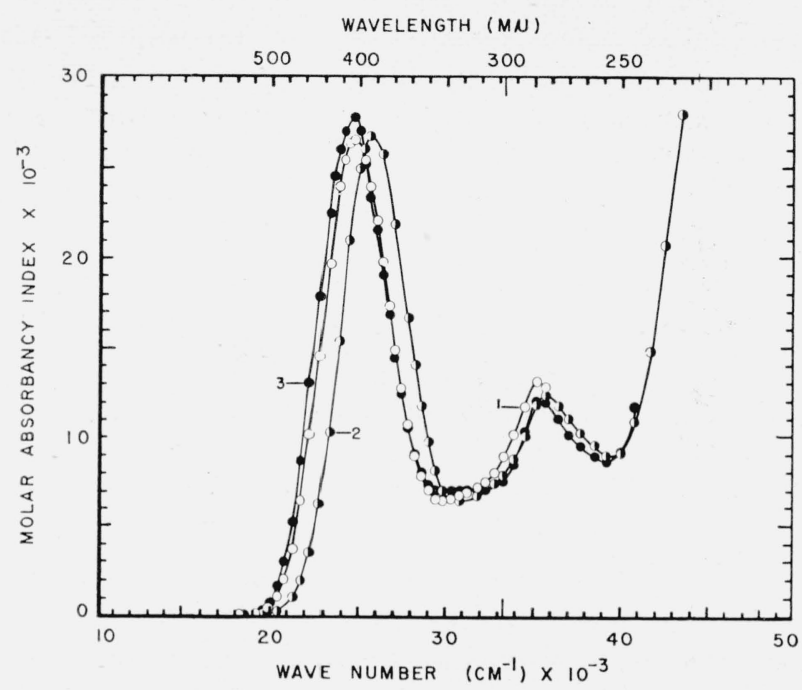

Figure 5. Effect of solvent upon molar absorbancy of bromphthalein magenta.

(1) Bromphthalein magenta $\mathrm{E}$ in benzene; (2) bromphthalein magenta $\mathrm{B}$ in cyclohexane; (3) bromphthalein magenta $\mathrm{E}$ in ethylene dichloride.

prevents measurements at wavelengths shorter than about $280 \mathrm{~m} \mu$, and there is no measurable absorption by the dye at wavelengths greater than about $530 \mathrm{~m} \mu$. Two maxima appear near $285 \mathrm{~m} \mu\left(35,100 \mathrm{~cm}^{-1}\right)$ and $405 \mathrm{~m} \mu\left(24,700 \mathrm{~cm}^{-1}\right)$, with the respective molar absorbancy indexes 13,000 and 26,500. A minimum is located near $335 \mathrm{~m} \mu\left(29,800 \mathrm{~cm}^{-1}\right)$, with the molar absorbancy index 6,450 .

Ramart-Lucas has given the absorbancy curve for the same compound in benzene [38] with the values for the logarithm of the molar absorbancy index plotted as ordinates. When the data for our best sample of bromphthalein magenta $\mathrm{E}$ were plotted on the same basis, the absorbancy was found to be greater than that reported by Ramart-Lucas. A sample of bromphthalein magenta $\mathrm{E}$ (specimen $A$ ), obtained from the first trial synthesis, was later shown by its chemical analysis and absorption spectrum to be contaminated with some other substance. The most likely impurity was tetrabromophenolphthalein, which could be formed by saponification of the potassium salt during the synthesis of bromphthalein magenta E. From the chemical analysis, it was calculated that specimen $A$ might contain 80 percent of bromphthalein magenta $E$ and 20 percent of tetrabromophenolphthalein. ${ }^{16}$ This assumption was checked spectrophotometrically; that is, when the absorption spectrum of specimen $A$ in benzene was compared with that of a solution that contained the calculated equivalents of bromphthalein magenta $\mathrm{E}$ and tetrabromophenolphthalein, the differences in the spectra were within experimental error. The lower values for the absorbancy indexes found by Ramart-Lucas may have been due to the presence of tetrabromophenolphthalein in the ethyl ester used by her. Unlike bromphthalein magenta, tetrabromophenolphthalein does not show visible reaction with organic bases in benzene, and it may therefore be regarded as an inert impurity when bromphthalein magenta is used as an indicator in benzene.

Cyclohexane.-The absorbancy curve obtained for $5 \times 10^{-5}-M$ bromphthalein magenta $\mathrm{B}$ in cyclohexane is given in figure 5 (curve 2). In the visible region, the curve is very similar to that obtained for an equimolar solution in benzene, except that it is slightly narrower and has shifted about 15 $\mathrm{m} \mu$ toward the ultraviolet. There are two maxi$\mathrm{ma}$, at the approximate positions $280 \mathrm{~m} \mu \quad(35,700$ $\left.\mathrm{cm}^{-1}\right)$ and $390 \mathrm{~m} \mu\left(25,600 \mathrm{~cm}^{-1}\right)$, with the respective molar absorbancy indexes 12,000 and 27,000. A strong absorption band near $45,000 \mathrm{~cm}^{-1}$ is also indicated. Two minima appear near $255 \mathrm{~m} \mu$ $\left(39,200 \mathrm{~cm}^{-1}\right)$ and $325 \mathrm{~m} \mu\left(30,800 \mathrm{~cm}^{-1}\right)$, with the respective indexes 9,000 and 6,400 . There was no evident deviation from Beer's law in the range of concentrations $1.25 \times 10^{-5}$ to $5 \times 10^{-5} \mathrm{M}$.

Ethylene dichloride.-The absorbancy curve obtained for $5 \times 10^{-5}-M$ bromphthalein magenta $\mathrm{E}$ in ethylene dichloride (curve 3, fig. 5) shows absorption and transmission bands in the same regions as those observed for an equimolar solution in benzene but with differences in intensity. The absorption bands found near $285 \mathrm{~m} \mu(35,100$ $\left.\mathrm{cm}^{-1}\right)$ and $405 \mathrm{~m} \mu\left(24,700 \mathrm{~cm}^{-1}\right)$ have the respective molar absorbancy indexes 12,000 and 28,000. That is, absorption of BPM-E in the ultraviolet region is slightly less in ethylene dichloride than in benzene, but in the visible region it is greater. However, these differences in the intensity of

\footnotetext{
16 A commercial specimen of the latter compound showed two narrow absorption bands in benzene near $284 \mathrm{~m} \mu$ and $292 \mathrm{~m} \mu$, with the respective molar absorbancy indexes 5,900 and 5,300.
} 
absorption are not great enough to exclude the possibility of their being due to some impurity in the commercial ethylene dichloride. Transmission bands appear near $255 \mathrm{~m} \mu\left(39,200 \mathrm{~cm}^{-1}\right)$ and $332 \mathrm{~m} \mu\left(30,100 \mathrm{~cm}^{-1}\right)$, with the respective absorbancy indexes 8,600 and 7,000. The latter transmission band is very flat, showing almost constant transmittancy in the range 310 to 340 $\mathrm{m} \mu$.

Other solvents. - Ramart-Lucas obtained nearly identical absorbancy curves for the ethyl ester of tetrabromophenolphthalein in benzene, chloroform, and dioxane [38]. For alcohol, she obtained an entirely different curve, with a narrow band of high intensity near $600 \mathrm{~m} \mu$. This is because the alcohol reacts as a base with the indicator, just as distilled water acts as a base with bromphenol blue to give the blue form of that indicator. ${ }^{17} \mathrm{~A}$ curve obtained for the potassium salt of bromphthalein magenta $\mathrm{E}$ or $\mathrm{B}$ in alcohol had a pattern very similar to that obtained by Ramart-Lucas and by the authors for the free indicator acid in alcohol, as well as to a curve obtained by her for a solution of the indicator in aqueous alcohol which contained sodium carbonate. Bromphthalein magenta gives a yellow solution in hydrocarbons and halogenated hydrocarbons other than those mentioned, as well as in very weak bases, such as ethyl ether and dioxane. Acetic acid and other

\footnotetext{
17 Ramart-Lucas does not attribute the change from yellow to blue to reaction with a base, as she does not recognize that alcohol and water have basic properties [38].
}

organic acids also give a yellow solution of the indicator. The effect of basic solvents will be discussed more fully in sections IV -8 and IV-10.

\section{Reaction of Bromphthalein Magenta with Ali- phatic Amines and Symmetrical Di- or Triaryl Derivatives of Guanidine in Benzene}

Tertiary aliphatic amines and $d i$ - or triarylguanidines. - The yellow color of bromphthalein magenta ( $\mathrm{E}$ or $\mathrm{B}$ ) in benzene is changed to a vivid magenta when an excess of a tertiary aliphatic amine or a symmetrical di- or triarylguanidine is added. Solutions in which only part of the indicator has been converted to the "salt" are orange, red, and intermediate tones, depending upon the relative concentrations of indicator and base. The different colors are very bright and easily distinguished by the unaided eye. Examples of organic bases that belong in this group are shown in figure 6 . Each of the solutions contained $4 \times 10^{-5}-M$ bromphthalein magenta $\mathrm{E}$, together with $5 \times 10^{-5}-M$ base $(1.25$ molar equivalents of the indicator) in a 1-cm absorption cell. In numerical order, the transmittancy curves represent the following solutions: (1) Indicator without added base; (2) $N$-ethylmorpholine; (3) 1, 2, 3-triphenylguanidine; (4) triisoamylamine; (5) tri- $n$-butylamine; (6) tri- $n$-amylamine; (7) triethylamine; (8) 1,3-diphenylguandine; (9) 1,3-di-o-tolylguanidine. Other bases not shown in this figure fell into the following respective orders: (1a) Tribenzylamine; (1b) pyridine; (3a) diethylbenzyl-

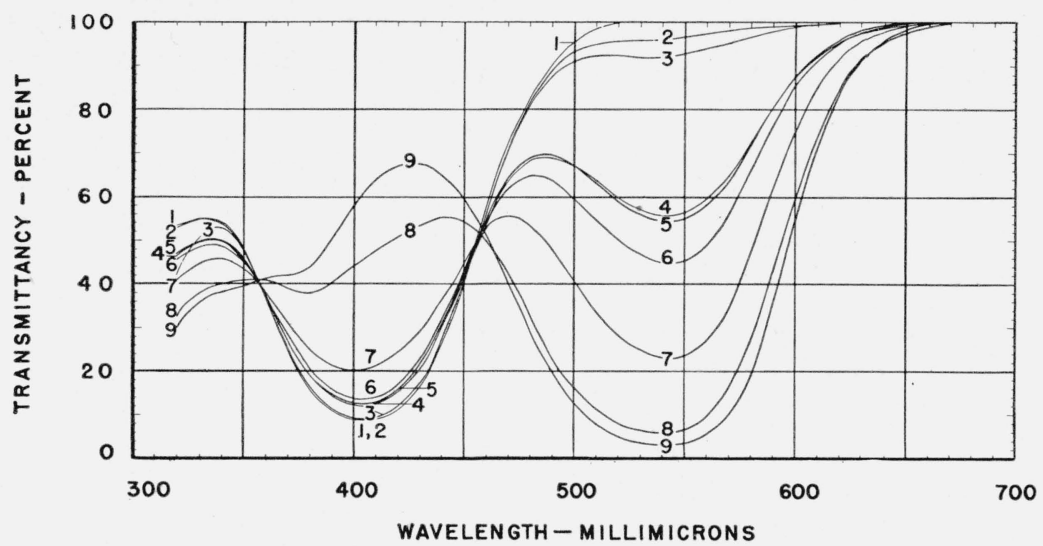

Figure 6. Transmittancy curves for bromphthalein magenta $\mathrm{E}, 4 \times 10^{-5} \mathrm{M}$ in benzene, in 1-cm cell, with $1.25 \mathrm{molar}$ equivalent of the following organic bases.

(1) None; (2) N-ethylmorpholine; (3) 1,2,3,-triphenylguanidine; (4) triisoamylamine; (5) tri- $n$-butylamine; (6) tri- $n$-amylamine; (7) triethylamine; (8) 1,3-diphenylguanidine; (9) 1,3-di-o-tolylguanidine. 
amıne; (5a) tri- $n$-propylamine; (10) di-m-xylylguanidine. Tribenzylamine and pyridine reacted only feebly. The studies were conducted primarily to determine the general pattern of behavior, and only a single measurement was made with each base. Because of this fact and also because traces of water, carbon dioxide, and in some cases other impurities may have been present in the bases, the quantitive relationships can be regarded as only provisional. However, the extent to which the various bases reacted with bromphthalein magenta gives some indication of their relative strengths. Comparison with the values for relative basic strengths in water is also of interest.

In table 2 are summarized values taken from the literature for the ionization constants in water of some of these bases. The values are expressed as $\mathrm{p} K_{b}$, that is, the negative logarithm of the ionization constant of the base. When one of these bases is dissolved in water, the following reactions may occur:

$$
\underset{\text { base }}{\mathrm{B}+\mathrm{H}_{2} \mathrm{O} \rightleftharpoons \mathrm{(a)}} \stackrel{(\mathrm{b})}{\rightleftharpoons} \mathrm{BHOH} \rightleftharpoons \mathrm{BH}^{+}+\mathrm{OH}^{-} .
$$

The ionization constant, $K_{b}$, refers to reaction $(1 b)$ and is formulated as follows: ${ }^{18}$

Therefore,

$$
K_{b}=\left[\mathrm{BH}^{+}\right]\left[\mathrm{OH}^{-}\right] /[\mathrm{BHOH}] .
$$

$$
\mathrm{p} K_{b}=\log \left\{[\mathrm{BHOH}] /\left[\mathrm{BH}^{+}\right]\left[\mathrm{OH}^{-}\right]\right\} .
$$

In table 2 , a smaller value for $\mathrm{p} K_{b}$ indicates a relatively large tendency for ions to form and vice versa. Authorities differ as to the role of reactions (a) and (b) in the evaluation of basic ionization constants. For example, the ionization constant is sometimes defined as $\left[\mathrm{BH}^{+}\right]\left[\mathrm{OH}^{-}\right] /[\mathrm{B}]$, or as $\left[\mathrm{BH}^{+}\right]\left[\mathrm{OH}^{-}\right] /\{[\mathrm{BHOH}]+[\mathrm{B}]\}$. Briegleb [55] has asserted that for the reaction of ammonia with water, reactions (a) and (b) are practically complete, and the ionization constant for ammonia is actually a measure of the equilibrium between $\left[\mathrm{NH}_{3} \mathrm{aq}\right]$ and $\left[\mathrm{NH}_{4} \mathrm{OH}_{\mathrm{aq}}\right]$. Moore and Winmill, on the other hand [12,56], assumed the reaction of $\mathrm{B}$ and $\mathrm{H}_{2} \mathrm{O}$ to give $\mathrm{BHOH}$, followed by ionization of $\mathrm{BHOH}$, with the respective equilibrium constants $K_{2}$ and $K_{1}$, and attempted the measurement of $K_{2}$ in order to arrive at a true value for $K_{1}$. In addition to the resultant uncertainty as to how:

\footnotetext{
${ }_{18}$ Concentrations are expressed as moles per liter; for simplification, con centrations are assumed to be identical with activities.
}

closely the "apparent" ionization constant in water approximates the "true" ionization constant, the low solubility of some of the organic bases in water is an obstacle to accurate measurements in this solvent [56]. Further considerations have been summarized by Hall [10].

TABLE 2.-Dissociation constants of some tertiary amines and arylguanidines in water a

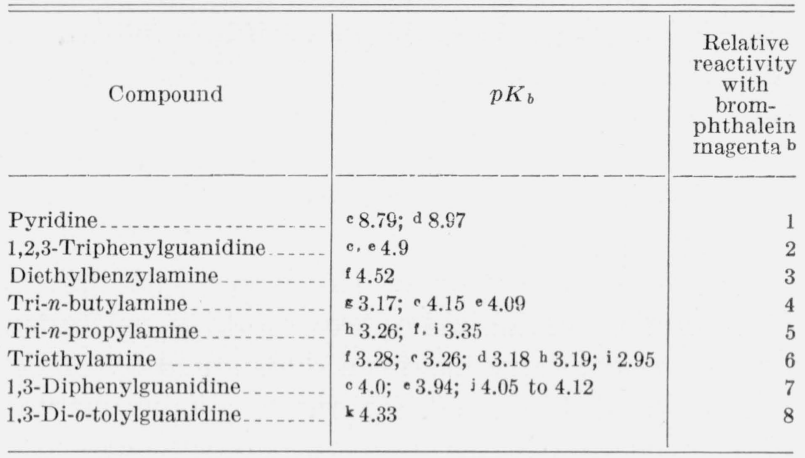

a In some cases, measurements were made in aqueous alcohol, and the value for aqueous solution was obtained by extrapolation. Values are for $25^{\circ} \mathrm{C}$, unless otherwise indicated.

b In benzene, in increasing order.

- N. F. Hall, J. Am. Chem. Soc. 52, 5115 (1930).

d H. T. S. Britton and W. G. Williams, J. Chem. Soc. (London) 796 (1935); values are for $18^{\circ} \mathrm{C}$.

- N. F. Hall and M. R. Sprinkle, J. Am. Chem. Soc. 54, 3469 (1932).

${ }^{\mathrm{J}} \mathrm{J}$. Kendall, Int. Crit. Tables VI, 260 (National Research Council, Washington, D. C., 1929).

g Damsgaard-Sørensen and A. Unmack, Z. phys. Chem. [A] 172, 389 (1935), value for $18^{\circ} \mathrm{C}$; Hall's valne 4.15 (sce footnote ${ }^{\circ}$ ) is believed by these authors to be in error.

h G. Bredig, Z. phys. Chem. 13, 289 (1894).

i T. S. Moore and T. F. Winmill, J. Chem. Soc. (London) 101, 1635 (1912).

j P. Walden and H. Ulich, Z. Elektrochem. 34, 25 (1928).

× L. Metz, Z. Elektrochem. 34, 292 (1928).

When an organic base, B, reacts with the indicator acid bromphthalein magenta, HOR, in benzene, the equation $\mathrm{B}+\mathrm{HOR} \rightleftharpoons \mathrm{BHOR}$ can be assumed for the initial reaction. There is strong evidence that in a partially substituted ammonium salt, "non-Coulombic interaction" occurs between the negative ion, and the proton of the positive ion in nonaqueous solvents $[28,57]$. In other words, a hydrogen bridge is formed between the cation and the anion. It seems to be fairly well established that in salts of the type BHOR the hydrogen bridge is strong, even in a solvent of relatively high dielectric constant. The cation and anion are also attracted to each other by a Coulombic force [57]. It therefore seems likely that in benzene the extent of dissociation of $\mathrm{BHOR}$ into $\mathrm{BH}^{+}$and $\mathrm{OR}^{-}$ions is negligible in many cases, though the molecule probably has a highly polar character. In benzene, consequently, 
the strength of the base is not to be measured in terms of the free ions formed, but by the extent to which B and HOR combine to form the salt BHOR. ${ }^{19}$ The basicity constant, $K_{b}$, may then be formulated in the following way:

$$
K_{b}=\left[{ }^{+} \mathrm{BH}^{-} \mathrm{OR}\right] /[\mathrm{B}][\mathrm{HOR}] .
$$

When the values for $\mathrm{p} K_{b}$ in water of the bases listed in table 2 are compared with the relative reactivities of the same bases with bromphthalein magenta in benzene, there seems to be a direct parallelism for the last five bases. The lack of conformity of the first three bases to this relationship may be due to the fact that their ionization constants in water are not a true measure of the extent of combination of the bases with water. The validity of ionization constants as an index of intrinsic basicity has been questioned by many writers, and the need for further experimental data, particularly for nonaqueous solvents, has been stressed. Furthermore, recent experiments have shown that in some instances steric factors interfere with the estimation of relative basicities $[59,60]$. For example, the basicities of tertiary aliphatic amines are lower than would be predicted on theoretical grounds [60].

There evidently is considerable uncertainty in the measurement of basicities in water. The sensitivity of the reaction of organic bases with bromphthalein magenta in benzene demonstrates the possible usefulness of this and other indicators for studying the effect of structure upon intrinsic basic strengths. In fact, the greater solubility

${ }^{19}$ According to some authorities, this should be called a "pseudo-salt." of these bases in benzene and the aprotic character of this solvent make the interpretation of data less equivocal than for parallel studies in water.

In figure 7 transmittancy curves are given to show the progressive reaction of $4 \times 10^{-5}-M$ bromphthalein magenta $E$ in benzene in a $1-\mathrm{cm}$ absorption cell with various increments of 1,3 diphenylguanidine. The smallest increment of base was 0.025 molar equivalent of the dye $\left(10^{-6}-M\right.$ base $)$, and with less than 3.75 molar equivalents $\left(1.5 \times 10^{-4}-M\right.$ base $)$ the "limiting" curve" was obtained. ${ }^{20}$ Two well-marked isosbestic points ${ }^{21}$ occur at approximately 355 and $460 \mathrm{~m} \mu$. The magenta form gives a broad absorption band with maximum near $540 \mathrm{~m} \mu$ and a transmission band near $420 \mathrm{~m} \mu$. Quantitative aspects of this reaction will be discussed more fully in a later section.

In figure 8 is shown the molar absorbancy curve of the salt that produces a magenta solution in benzene. The compositions of the solutions that gave the three curves shown were as follows: (1) bromphthalein magenta $\mathrm{B}, 5 \times 10^{-5} \mathrm{M}$, with $5 \times 10^{-3}-M$ triethylamine (molar ratio, $1: 100$ ); (2) bromphthalein magenta $\mathrm{E}, 5 \times 10^{-5} \mathrm{M}$, with $5 \times 10^{-4}-M 1$, 3-di-c-tolylguanidine (molar ratio, $1: 10)$; (3) bromphthalein magenta $\mathrm{B}, 2.5 \times 10^{-5} \mathrm{M}$, with $2.5 \times 10^{-4}-M$ ditolylguanidine (molar ratio, 1:10). The concentration of base was in each case

\footnotetext{
${ }^{20}$ So termed because continued addition of base produced no further change in the transmittancy curve, showing that reaction of the indicator with the base had reached completion.

${ }^{21}$ Isosbestic means "of equal extinction" [61]. Well-marked isosbestic points are interpreted as showing that two and only two colored species are present in the solution under examination. Isosbestic points at the same spectral regions may be noted in figure 6 .
}

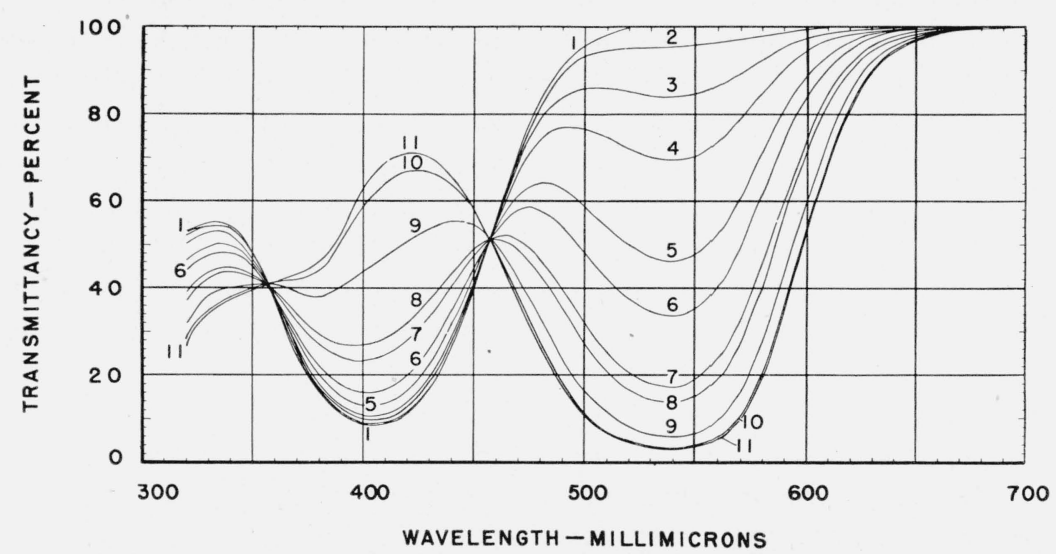

Figure 7. Transmittancy curves for bromphthalein magenta $\mathrm{E}, 4 \times 10^{-5} \mathrm{M}$ in benzene, in 1 -cm cell, with various concentrations of 1,3-diphenylguanidine expressed as molar equivalents of the indicator.

(1) None; (2) 0.025 ; (3) 0.0625 ; (4) 0.125 ; (5) 0.25 ; (6) 0.375 ; (7) 0.625 ; (8) 0.75 ; (9) 1.25 ; (10) 2.5 ; (11) 3.75 (limiting curve). 


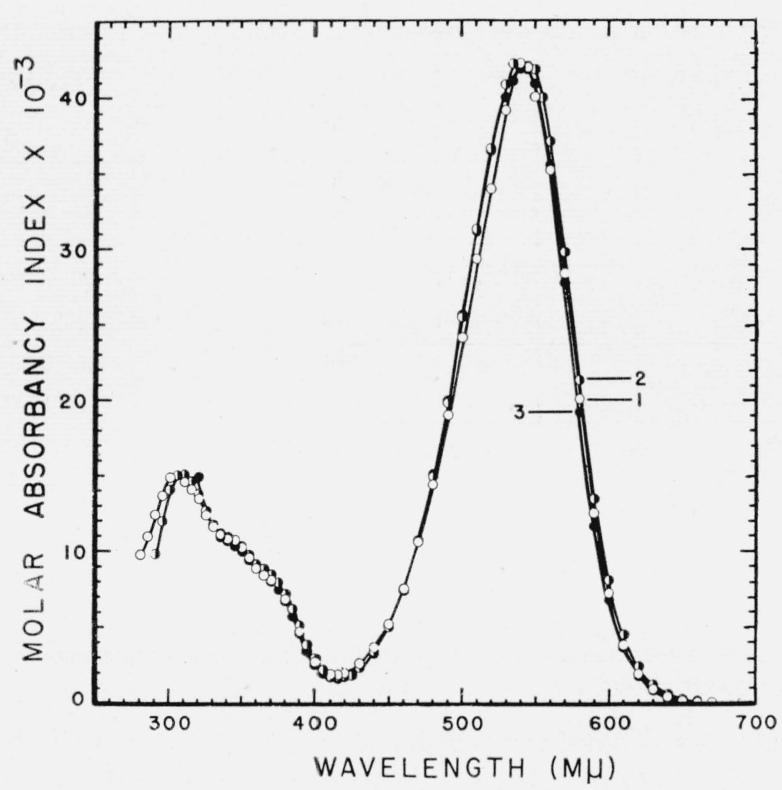

FIgURE 8. Typical molar absorbancy curves for products from complete reaction of bromphthalein magenta with tertiary aliphatic amines or 1,3-diarylguanidines in benzene.

(1) Bromphthalein magenta $\mathrm{B}, 5 \times 10^{-5} M$, plus 100 molar equivalents of triethylamine; (2) bromphthalein magenta $\mathrm{E}, 5 \times 10^{-5} \mathrm{M}$, plus 10 molar equivalents of 1,3-di-o-tolylguanidine; (3) bromphthalein magenta B, $2.5 \times 10^{-5}$ $M$, plus 10 molar equivalents of 1,3-di-o-tolylguanidine.

greater than that required to produce the limiting curve. There are two absorption bands in the spectrum, one near $305 \mathrm{~m} \mu\left(a_{M}=\right.$ approximately 15,000$)$ and a second near $540 \mathrm{~m} \mu\left(a_{M}=\right.$ about 42,000$)$, together with evidence of overlapping bands in the region 340 to $370 \mathrm{~m} \mu$ and a transmission band near $415 \mathrm{~m} \mu\left(a_{M}=2,000\right)$. When these curves were obtained, the absorption cells available were not short enough to permit measurements of high accuracy near $540 \mathrm{~m} \mu$. This is probably the cause of the slight differences among the curves in this region. In order to conserve base, and also because the effect of the indicator upon the spectral absorption of the base is not known, benzene was used in the comparison cell instead of a solution containing the base. This would account for the small differences in the region 280 to $330 \mathrm{~m} \mu$. It is evident that the change in absorption is small when (1) bromphthalein magenta B is used instead of bromphthalein magenta E; (2) ditolylguanidine is used instead of triethylamine; or (3) the concentration of indicator is altered to a limited degree.

It should be mentioned-and this statement applies to all of the reactions of bromphthalein magenta with bases - that in all of the experiments performed, the color change produced by adding one of these bases to this indicator in benzene appeared to be instantaneous. The reaction is exothermic, as shown by the fact that heating the solution causes fading toward the original yellow, and cooling produces a deepening toward magenta. The heat of reaction appears to be smaller for the reaction of bromphthalein magenta with a tertiary amine than for its reaction with primary or secondary amines.

Secondary aliphatic amines. - The yellow color of bromphthalein magenta in benzene is changed to purple-blue ("cornflower" blue) when an excess of a secondary aliphatic amine is added. ${ }^{22}$ Solutions that contain free indicator acid mixed with some of its salt with a secondary amine are green or another intermediate color, depending upon the relative amounts of the yellow and blue substances. This is in striking contrast to the behavior of bromphthalein magenta with tertiary amines, which was described in the preceding section. The appearance of the transmittancy curves is illustrated in figure 9 . The concentrations of indicator and of base were the same as those that gave the curves in figure 6 ; that is, each solution contained $4 \times 10^{-5}-M$ bromphthalein magenta $\mathrm{E}$ in benzene together with $5 \times 10^{-5}-M$ amine (molar ratio, $1: 1.25$ ) in a 1-cm absorption cell. Ignoring for the moment curves 2 to 13 , the curves represent solutions containing the following bases: (1) None; (14) di- $n$-propylamine; (15) di- $n$-amylamine; (16) di- $n$-butylamine; (17) diethylamine; (18) diisoamylamine; (19) dicyclohexylamine; (20) piperidine. Other secondary amines not given in the figure, in their relative order of apparent strength, are (17a) di- $n$-octylamine; (19a) 2,3dimethylpiperidine; (19b) 2-methylpiperidine. Dibenzylamine showed negligible reaction, and di-2ethylhexylamine was much less reactive than dipropylamine. These relative orders are decidedly provisional. In the first place, it is evident from figure 9 that the transmittancies near 575 $\mathrm{m} \mu$, the region of maximum absorption, are too close to zero for precise measurements. At the time these measurements were made the shortest available absorption cell was $1 \mathrm{~cm}$ long, and it was desired to use concentrations of indicator and

22 This statement should be qualified as referring to daylight illumination and to relatively dilute solutions or to thin layers of more concentrated solutions. By incandescent light or in thick layers, the color is dark ed. This behavior resembles the so-called "dichromatism" of some of the sulfonephthalein indicators, including bromphenol blue [62, 63]. 


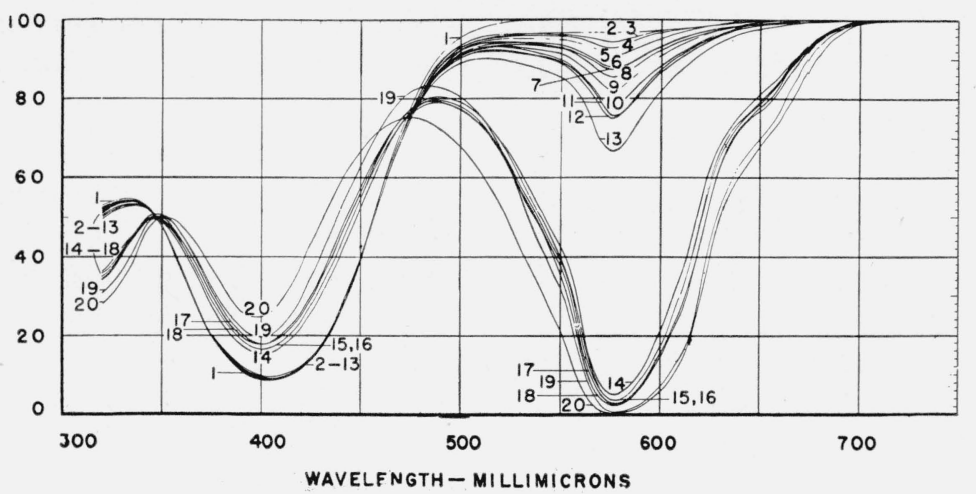

FigURE 9. Transmittancy curves for bromphthalein magenta $\mathrm{E}, 4 \times 10^{-5} \mathrm{M}$ in benzene, in 1-cm cell, with $1.25 \mathrm{molar}$ equivalent of the following organic bases.

(1) None; (2) benzylamine; (3) isobutylamine; (4) o-aminodicyclohexyl; (5) $n$-propylamine; (6) isoamylamine; (7) $n$-butylamine; (8) sec-butylamine; (9) $n$-amylamine; (10) isopropylamine; (11) cyclohexylamine; (12) $n$-octylamine; (13) $n$-heptylamine; (14) di- $n$-propylamine; (15) di- $n$-amylamine; (16) di- $n$-butylamine; (17) diethylamine; (18) diisoamylamine; (19) dicyclohexylamine; (20) piperidine.

amine that would permit comparisons with other experiments. Variations of a few tenths of a degree in the temperature during measurement are also more serious than in the case of measurements involving tertiary amines. Although the temperature of the room was kept constant without much difficulty, heating of the solutions by the adjacent light source presented a problem. For these reasons and also because several of the amines were available only in small quantities, purification was dispensed with in some cases. However, for the solution that contained di- $n$ butylamine, the same transmittancy curve was obtained irrespective of whether the commercial high-grade amine was used without treatment or after drying and redistilling. It was at first supposed that a trace of water in the secondary amine might be the explanation for a blue rather than a magenta color, but no such effect of water could be demonstrated.

The curves have common intersections near 345 to $350 \mathrm{~m} \mu$ and 470 to $475 \mathrm{~m} \mu$, with the exception of the curve for the solution that contained dicyclohexylamine. The latter curve intersects the curve for the indicator at approximately $480 \mathrm{~m} \mu$. The deviation may be characteristic of this amine or may be evidence of an impurity in the sample.

The values for $\mathrm{p} K_{b}$ in water for some secondary aliphatic amines are given in table 3 . When these values are compared with those given in table 2 for corresponding tertiary aliphatic amines, the following facts are illustrated: 1, The secondary aliphatic amines, with lower values for $\mathrm{p} K_{b}$, show greater basicity in water; 2 , variations in basicity among secondary aliphatic amines are relatively slight; 3 , quantitative comparisons of the basicity of secondary amines are difficult, because of the variation in the values of $\mathrm{p} K_{b}$ for a given amine reported by different investigators.

TABLE 3.-Dissociation of some secondary aliphatic amines in water s

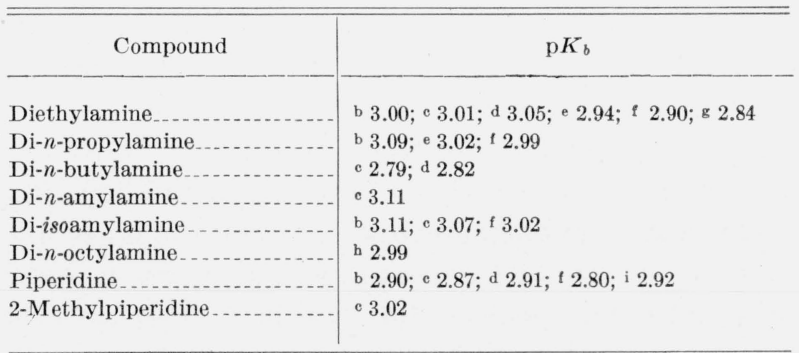

a In some cases, measurements were made in aqueous alcohol, and the value for aqueous solution was obtained by extrapolation. Values are for $25^{\circ} \mathrm{C}$ unless otherwise indicated.

b J. Kendall, Int. Crit. Tables VI, 260 (National Research Council, Washington, D. C., 1929).

- N. F. Hall and M. R. Sprinkle, J. Am. Chem. Soc. 54, 3469 (1932).

d N. F. Hall, J. Am. Chem. Soc. 52, 5115 (1930).

e T. S. Moore and T. F. Winmill, J. Chem. Soc. (London) 101, 1635 (1912).

f G. Bredig, Z. phys. Chem. 13, 289 (1894).

g H. T. S. Britton and W. G. Williams, J. Chem. Soc. (London), 796 (1935); values are for $18^{\circ} \mathrm{C}$.

h C. W. Hoerr, M. R. McCorkle, and A. W. Ralston, J. Am. Chem. Soc. 65, 328 (1943); probable accuracy \pm 0.01 .

i T. S. Moore, J. Chem. Soc. London 91, 1373 (1907).

The transmittancy curves in figure 10 show the reaction of bromphthalein magenta $\mathrm{E}$ in benzene with progressively increasing amounts of piperidine. The curves correspond to $5 \times 10^{-5}-M$ indicator in a 5-mm cell with the following concentrations of piperidine, expressed as molar equivalents of dye: (1) None; (2) 0.1 ; (3) 0.3 ; (4) 
0.5 ; (5) 1.0 ; (6) 10 (limiting curve). In other words, the reaction was complete upon or before the addition of $5 \times 10^{-4}-M$ piperidine. Three isosbestic points at 295,345 , and $475 \mathrm{~m} \mu$ are evident. ${ }^{23}$ The sensitivity of the reaction is noteworthy. Quantitative aspects will be referred to in a later section.

The molar absorbancy curve of the "salt" formed by the complete reaction of bromphthalein magenta with a secondary aliphatic amine in benzene is shown in figure 11 . Curve 1 corresponds to $2.5 \times 10^{-5}-M$ BPM-E with 40 molar equivalents of diethylamine, and curve 2 corresponds to $5 \times 10^{-5}-M$ BPM-E with 10 molar equivalents of piperidine. In both cases an excess of base was present. These two curves are virtually superimposed and except in the nearly vertical portions, where measurements are less precise, values for the two solutions are almost identical. The absorbancy curve shows a very strong band near $575 \mathrm{~m} \mu \quad\left(a_{M}=\right.$ approximately $90,000)$ and two weaker bands near $315 \mathrm{~m} \mu\left(a_{M}=\right.$ $18,000)$ and $395 \mathrm{~m} \mu\left(a_{M}=\right.$ about 10,000$)$, with transmission bands in the regions $360 \mathrm{~m} \mu\left(a_{M}=6,000\right)$ and $450 \mathrm{~m} \mu \quad\left(a_{M}=\right.$ less than 1,000). When this absorbancy curve is compared with that for the salt that is formed by reaction of bromphthalein magenta with a tertiary amine (see fig. 8), the striking difference between the two absorbancy curves, that correspond respectively to purpleblue and magenta solutions in benzene, is readily perceived. The difference would be even more apparent had the two graphs been plotted with the same relation between ordinates and abscissas. The maximum molar absorbancy for the purpleblue solution is more than twice that for the magenta solution. The main band obtained for the former solution is very steep and narrow, while that for the magenta solution is relatively much broader. Differences in the spectral region, 280 to $400 \mathrm{~m} \mu$, are also obvious. The differences in the reactions of secondary and tertiary amines with bromphthalein magenta in benzene will be discussed more fully in sections IV -8 and IV -10 .

Primary aliphatic amines.-The color produced by adding an excess of a primary aliphatic amine to bromphthalein magenta in benzene is a redpurple, sometimes referred to as "plum color."

\footnotetext{
${ }^{23}$ Piperidine was not present in the comparison cell, but even at the highest concentration used in these experiments its effect upon the transmittancy of benzene is only about 0.3 percent at $280 \mathrm{~m} \mu$ and less at longer wavelengths.
}

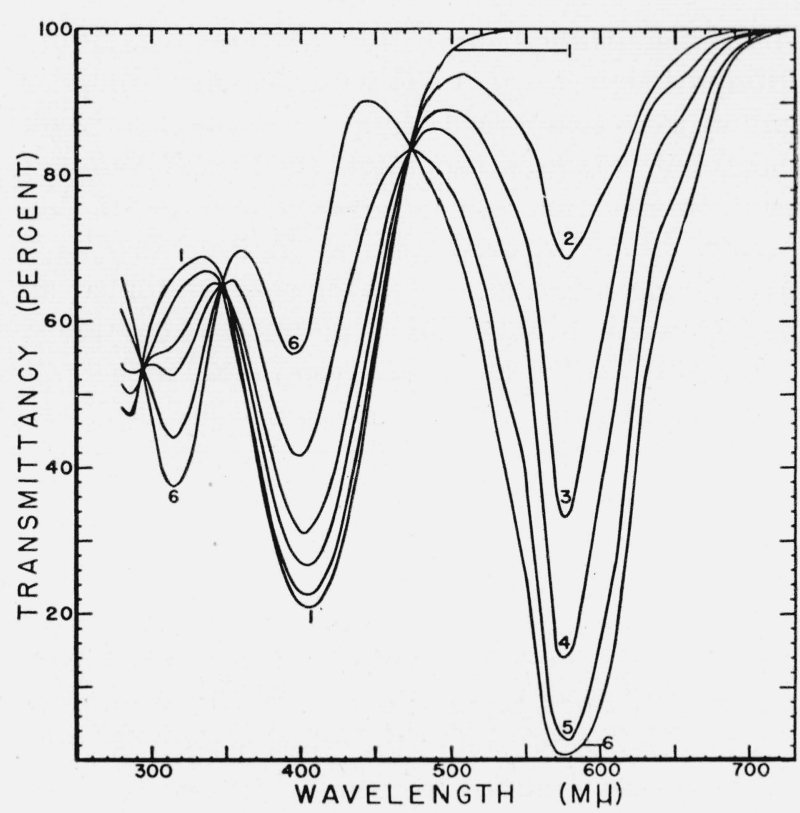

Figure 10. Transmittancy curves for bromphthalein magenta $\mathrm{E}, 5 \times 10^{-5} \mathrm{M}$ in benzene, in 5 - $\mathrm{mm}$ cell, with various concentrations of piperidine expressed as molar equivalents of the indicator.

(1) None; (2) 0.1 ; (3) 0.3 ; (4) 0.5 ; (5) 1.0 ; (6) 10 (limiting curve).

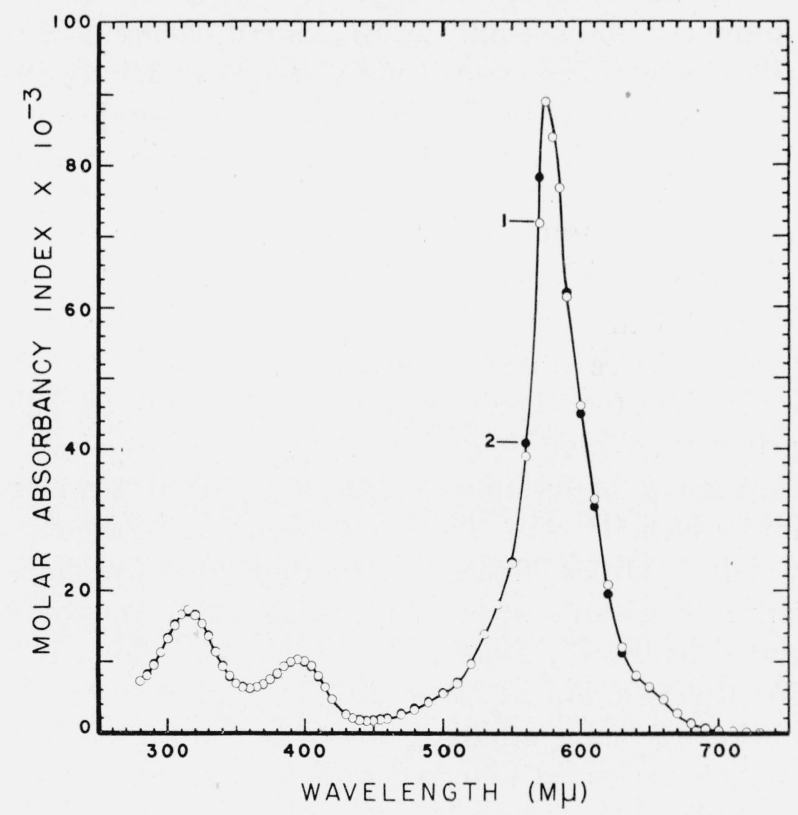

Figure 11. Typical molar absorbancy curves for products of complete reaction of bromphthalein magenta $E$ in benzene with secondary aliphatic amines.

(1) Bromphthalein magenta $\mathrm{E}, 2.5 \times 10^{-5} \mathrm{M}$, plus 40 molar equivalents of diethylamine; (2) bromphthalein magenta $\mathrm{E}, 5 \times 10^{-5} \mathrm{M}$, plus 10 molar equivalents of piperidine. 
The transmittancy curves for solutions containing bromphthalein magenta $\mathrm{E}$ with primary aliphatic amines were temporarily ignored in the discussion of figure 9. On first inspection, the primary amines seem to resemble the secondary amines in behavior, except in producing a smaller effect at a given concentration. The curves assembled in figure 9 were obtained for solutions that contained $4 \times 10^{-5}-M$ BPM-E in a $1-\mathrm{cm}$ cell, with 1.25 molar equivalent of the amine $\left(5 \times 10^{-5} M\right)$. In the order of increasing reactivity with bromphthalein magenta, the amines were: (2) Benzylamine; (3) isobutylamine; (4) o-aminodicyclohexyl; (5) n-propylamine; (6) isoamylamine; (7) n-butylamine; (8) sec.-butylamine; (9) n-amylamine; (10) isopropylamine; (11) cyclohexylamine; (12) n-octylamine; and (13) n-heptylamine. 2 -Amino- $n$-octane was in the relative position (9a). It should be emphasized that quantitative relationships are only provisional, because the experiments were of an exploratory nature, and minor variations in temperature had a noticeable effect upon the values obtained.

Data for values of $\mathrm{p} K_{b}$ in water for some primary aliphatic amines are presented in table 4 . The lack of agreement in the values of $\mathrm{p} K_{b}$ for a given amine are due not only to the varied nature of the experimental procedures used by different investigators but also to diversity in the assumptions made in calculations from the data. It is plainly evident, however, that the primary aliphatic amines exhibit lower basicity in water than the corresponding secondary amines. If the values cited first in table 4 are used as the basis for comparison, there appears to be a trend toward greater ionization for the homologous series of normal primary aliphatic amines from $n$-propylamine through $n$-heptylamine, and a reversal toward lower basicity with further increase in molecular weight. Other effects of structure upon the ionization constants of primary amines in water were noted by Bredig (footnote d, table 4). The data for the reaction of bromphthalein magenta with various primary amines in benzene show a smaller tendency toward reaction than that exhibited by secondary amines. Furthermore, as $\mathrm{p} K_{b}$ for water increases, there is seemingly a decrease in the reactivity in benzene. This is the reverse of the relationship found for five of the tertiary amines and arylguanidines. The data for the reactions of different primary amines with brom- phthalein magenta in benzene are too provisional to warrant detailed speculation, but it seems likely that the reaction of the amines with water and with bromphthalein magenta may be obscured by lack of knowledge as to the real extent of hydration and ionization of these bases in water and of any self-association of the amine.

TABLE 4.-Dissociation constants of some primary aliphatic amines in water a

\begin{tabular}{|c|c|c|}
\hline & Compound & $\mathrm{p} K_{b}$ \\
\hline & Methylamine..... & b $3.36 ;$ c $3.40 ;$ d, $\circ 3.30 ;$ f 3.44 \\
\hline & Ethylamine....... & - $3.34 ; \mathrm{d} 3.13 ; \bullet 3.25 ;{ }^{\mathrm{f}} 3.40$ \\
\hline (3) & $n$-Propylamine & • $3.41 ;$ d $3.32 ;$ • 3.33 \\
\hline (5) & $n$ Butylamine $\ldots$ & g,h 3.39 \\
\hline \multirow{2}{*}{ (7) } & $n$-Amylamine & E $3.37 ;$ : 3.33 \\
\hline & $n$-Hexylamine & g 3.36 \\
\hline (11) & $n$-Heptylamine & s 3.34 \\
\hline \multirow{8}{*}{ (10) } & $n$-Octylamine. & g 3.35 \\
\hline & $n$-Nonylamine $\ldots \ldots$ & g 3.36 \\
\hline & $n$-Decylamine & g 3.36 \\
\hline & $n$-Undecylamine & g 3.37 \\
\hline & $n$-Dodecylamine................ & g 3.37 \\
\hline & $n$-Tridecylamine. & g 3.37 \\
\hline & $n$-Tetradecylamine & g 3.38 \\
\hline & $n$-Pentadecylamine & g 3.39 \\
\hline (8) & Isopropylamine & с $3.37 ; \bullet 3.28$ \\
\hline (2) & Isobutylamine . . . & g $3.57 ;$ с $359 ;$ e 3.51 \\
\hline (6) & sec-Butylamine & - $3.44 ; \circ 3.34$ \\
\hline (4) & Isoamylamine & с, в $3.40 ;$ ॰ $3.30 ; ³ .33$ \\
\hline (9) & Cyclohexylamine & i $3.31 ;$ j 3.80 \\
\hline (1) & Benzylamine & \$ $4.63 ; \bullet 4.70 ; \bullet 4.61$ \\
\hline
\end{tabular}

a In some cases, measurements were made in aqueous alcohol, and the value for aqueous solution was obtained by extrapolation. Values are for $25^{\circ} \mathrm{C}$ unless otherwise indicated. The numbers to the left of the names of the compounds indicate the apparent order of increasing reactivity with bromphthalein magenta $E$ in benzene.

b H. S. Harned and B. B. Owen, J. Am. Chem. Soc. 52, 5079 (1930).

- J. Kendall, Int. Crit. Tables VI, 260 (National Research Council, Washington, D. C., 1929).

d T. S. Moore and T. F. Winmill, J. Chem. Soc. (London) 101, 1635 (1912). - G. Bredig, Z. phys. Chem. 13, 289 (1894).

` H. T. S. Britton and W. G. Williams, J. Chern. Soc. (London) 796 (1935); values are for $18^{\circ} \mathrm{C}$.

g C. W. Hoerr, M. R. McCorkle, and A. W. Ralston, J. Am. Chem. Soc. 55, 328 (1943); probable accuracy, \pm 0.01 unit.

h L. D. Goodhue and R. M. Hixon, J. Am. Chem. Soc. 56, 1329 (1934).

j N. F. Hall and M. R. Sprinkle, J. Am. Chem. Soc. 54, 3469 (1932).

i A. Waksmundzki, Roczniki Chem. 18 i, 865 (1938), through Chem. Abstracts 33, 6688 (1939); values are for $18^{\circ}$ to $20^{\circ} \mathrm{C}$.

k W. H. Carothers, C. F. Bickford, and G. J. Hurwitz, J. Am. Chem. Soc. 49, 2908 (1927).

Figure 12 contains curves for the reaction of $5 \times 10^{-5}-M$ bromphthalein magenta $\mathrm{E}$ with successively greater quantities of $n$-heptylamine in a 5-mm cell. The curves show the effects of the following concentrations of the amine, expressed as molar equivalents of the indicator: (1) none; (2) 1 ; (3) 2 ; (4) 5 ; (5) 20 ; (6) 80 (limiting curve). At the higher concentrations of amine, it becomes apparent that the main absorption band has two 
maxima, one near $540 \mathrm{~m} \mu$ and the other near 575 $\mathrm{m} \mu$. These are the wavelengths of maximum absorbancy for solutions of bromphthalein magenta with tertiary amines and secondary amines, respectively. In figure 12 three well-defined isosbestic points are evident in the spectral region 290 to $295 \mathrm{~m} \mu, 345$ to $350 \mathrm{~m} \mu$, and 465 to $470 \mathrm{~m} \mu$. Two single absorption bands occur near $315 \mathrm{~m} \mu$ and $390 \mathrm{~m} \mu$, and transmission bands near 335 and $440 \mathrm{~m} \mu$. The ratio of the molar absorbancy indexes at $575 \mathrm{~m} \mu$ and $540 \mathrm{~m} \mu$ is not constant but falls from nearly 2.5 for one molar equivalent of base to approximately 1.2 for five or more molar equivalents. Dimerization of the base or formation of still larger aggregates at the higher concentrations may be a partial explanation for this behavior. The absorbancy curve is affected by fluctuations of only a few tenths of a degree centigrade, and when the temperature is lowered there is some evidence of a strengthening of the band near $575 \mathrm{~m} \mu$ at the expense of the band near $540 \mathrm{~m} \mu$.

In figure 13 are shown typical molar absorbancy curves for the salts formed by the complete reaction of bromphthalein magenta with three different primary amines in benzene. These curves were obtained for $5 \times 10^{-5}-M$ bromphthalein magenta $\mathrm{E}$ with the following amines: (1) Cyclohexylamine (100 molar equivalents); (2) $n$-heptylamine (80 molar equivalents); (3) $n$ octylamine ( 80 molar equivalents). The general pattern of the absorption bands is the same throughout the spectrum for the three salts, and the intensity of absorbancy is practically the same in the region 280 to $420 \mathrm{~m} \mu$. In the main band the total intensity differs for the three substances, and the ratios of the molar absorbancy indexes of the partial bands at 575 and $540 \mathrm{~m} \mu$ are approximately $1.25,1.17$, and 1.14 for salts 1,2 , and 3 , respectively. The differences do not appear to be due to instability, for solutions that contained up to 100 molar equivalents of any one of these bases gave constant transmittancy values for at least 4 hours.

\section{Preparation and Absorption Spectra of Tet- raalkylammonium Salts of Bromphthalein Ma- genta}

Preparation.-To prepare the tetraethylammonium salt of bromphthalein magenta E, a solution of bromphthalein magenta $\mathrm{E}$ in benzene was shaken mechanically for 10 minutes with an ap-

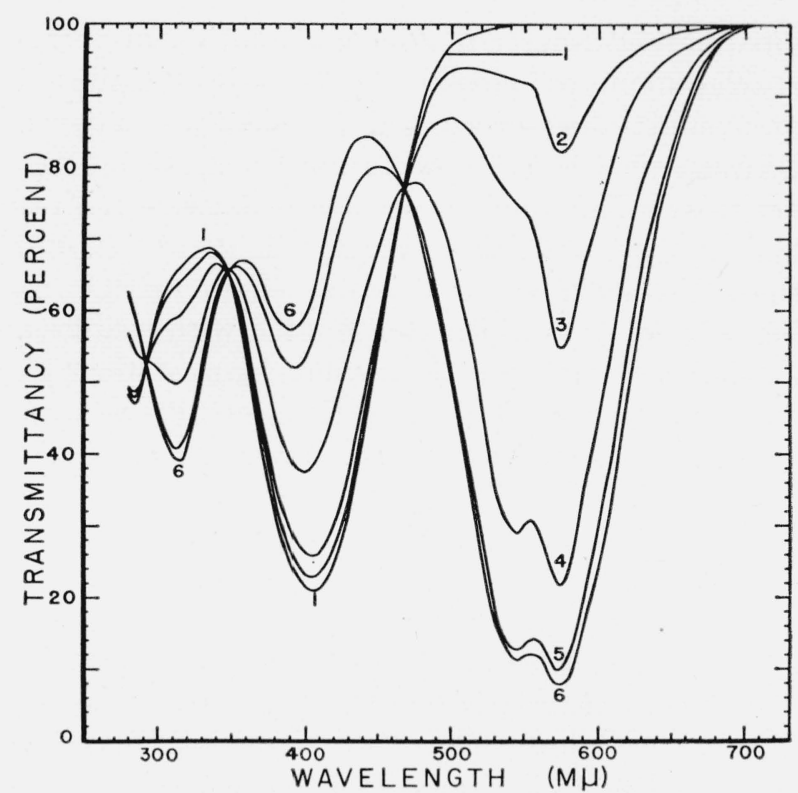

Figure 12. Transmittancy curves for bromphthalein magenta $E, 5 \times 10^{-5} M$ in benzene, in a $5-\mathrm{mm}$ cell, with various concentrations of n-heptylamine expressed as molar equivalents of the indicator.

(1) None; (2) 1 ; (3) 2; (4) 5; (5) 20; (6) 80 (limiting curve).

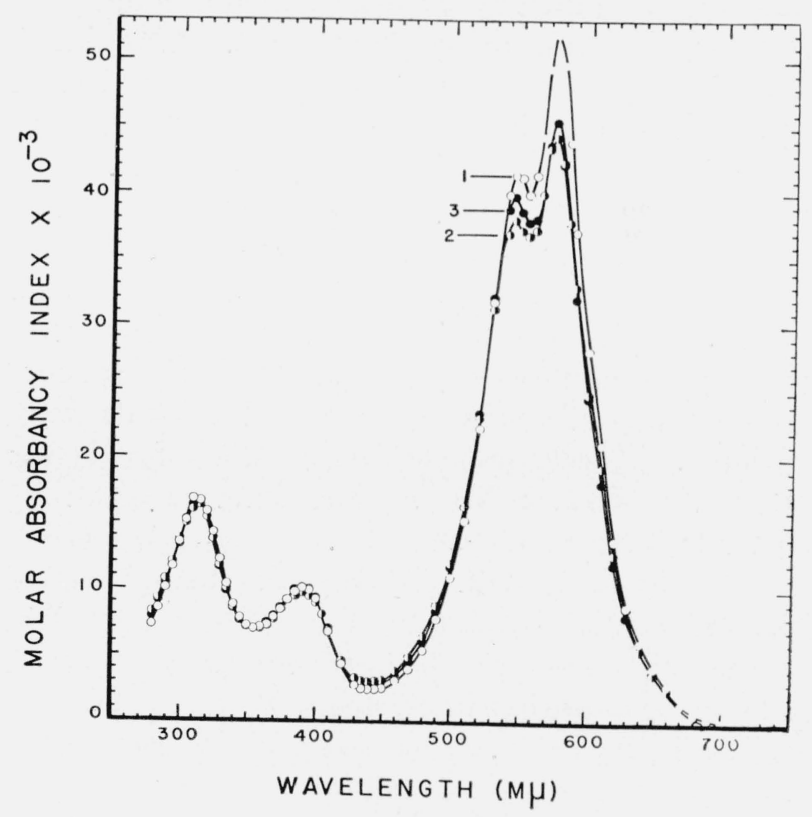

Figure 13. Typical molar absorbancy curves for products of complete reaction of bromphthalein magenta $E, 5 \times 10^{-5}$ $M$ in benzene, with primary aliphatic amines.

Amine added and its concentration in molar equivalents of the indicator: (1) Cyclohexylamine, 100; (2) $n$-heptylamine, 80 ; (3) $n$-octylamine, 80. 
proximately 2-percent aqueous solution of tetraethylammonium hydroxide. The salt precipitated as a shiny dark-green solid, which was filtered with suction after decantation of most of the benzene. The solid was extracted twice with a little hot benzene, the benzene was decanted, and the moist residue was dried at about $60^{\circ} \mathrm{C}$. A benzene solution of the salt reacted with cellulose, as shown by the fact that when poured through dry filter paper the first portions of filtrate were green instead of the original blue. Moreover, a sample of the solid salt was decomposed to a gummy orange solid upon extraction with benzene in a Soxhlet apparatus. It is reasonable to suppose that this decomposition was caused by the strong tendency of quaternary ammonium bases, like other strong bases, to combine with and even dissolve cellulose [64]. The reaction may be visualized as an alcoholysis of the salt, with cellulose playing the role of an alcohol.

Analysis of purified salt: Calculated for $\mathrm{C}_{30} \mathrm{H}_{33} \mathrm{O}_{4} \mathrm{NBr}_{4}$ : C, 45.5; H, 4.2; N, 1.8; Br, 40.4. Found: C, 45.2; H, 4.6; N, 1.6 ; Br, 40.6.

In the preparation of the tetra- $n$-butylammonium salt of bromphthalein magenta B, a benzene solution of bromphthalein magenta $B$ was shaken mechanically with an aqueous solution of tetra- $n$-butylammonium hydroxide. The latter solution was the filtrate from the reaction of aqueous tetra- $n$-butylammonium iodide with an excess of freshly precipitated silver oxide. The tetrabutylammonium salt remained dissolved in the benzene layer, giving it a deep-blue color, but was precipitated upon the addition of petroleum ether. When the precipitate was redissolved in benzene and allowed to crystallize slowly at room temperature, large bronze-colored crystals were formed. Analysis: Calculated for $\mathrm{C}_{40} \mathrm{H}_{53} \mathrm{O}_{4} \mathrm{NBr}_{4}$ : C, $51.6 ; \mathrm{H}, 5.7 ; \mathrm{N}, 1.5 ; \mathrm{Br}, 34.3$. Found: C, $51.5 ; \mathrm{H}, 5.8 ; \mathrm{N}, 1.4 ; \mathrm{Br}, 34.5$.

Absorption spectra.-Molar absorbancy curves for the tetraethylammonium salt of bromphthalein magenta $\mathrm{E}$ and for the tetra- $n$-butylammonium salt of bromphthalein magenta $\mathrm{B}$ in benzene are shown in figure 14 . Curves $1 \mathrm{~B}$ to $6 \mathrm{~B}$ are for the butyl derivative, at the following successive molar concentrations: $10^{-4}, 4 \times 10^{-5}, 2 \times 10^{-5}, 10^{-5}$, $5 \times 10^{-6}$, and $2 \times 10^{-6}$. The less concentrated solutions were prepared by dilution of the $10^{-4}-M$ stock solution. Curves $4 \mathrm{E}$ to $6 \mathrm{E}$ are for the ethyl derivative at the following successive molar con- centrations: $10^{-5}, 5 \times 10^{-6}$, and $2 \times 10^{-6}$. The stock solution in this case was $10^{-5} \mathrm{M}$, which is about the limit of the solubility of the ethyl derivative in benzene. Both salts possess a strong absorption band near 600 to $605 \mathrm{~m} \mu(16,670$ to $16,530 \mathrm{~cm}^{-1}$ ) and three shallow absorption bands in the ultraviolet region. However, the molar absorbancy at $605 \mathrm{~m} \mu$ does not adhere to Beer's law but decreases with dilution, falling from the maximum absorbancy index 82,000 for $1 \mathrm{~B}$ to the index 51,000 for $6 \mathrm{~B}$. The indexes at $605 \mathrm{~m} \mu$ for $4 \mathrm{E}$ and $6 \mathrm{E}$ are, respectively, 62,500 and 42,000 .

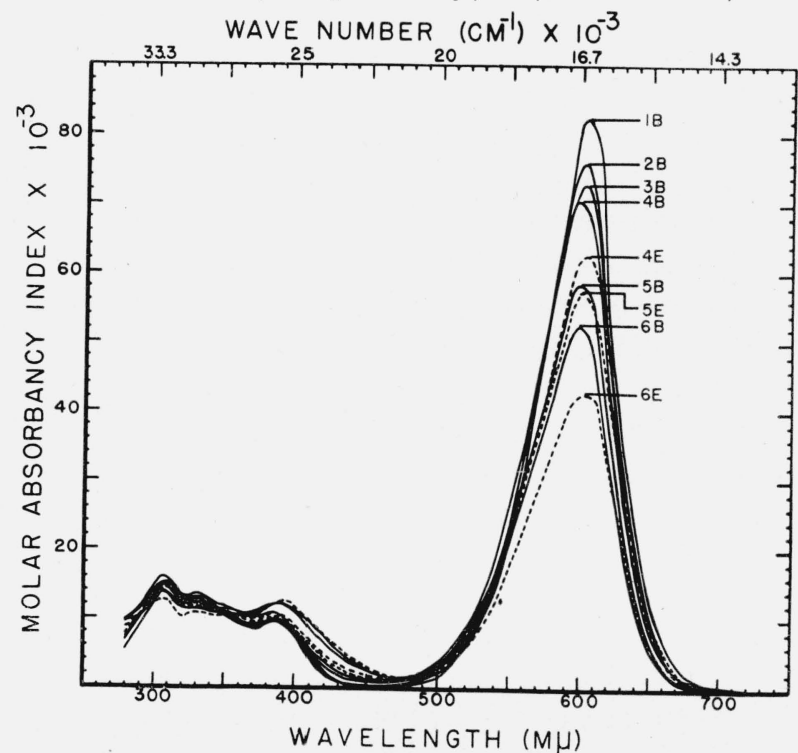

Figure 14. Molar absorbancy curves for tetraalkylammonium salts of bromphthalein magenta in benzene at various concentrations.

(1B to $6 \mathrm{~B}$ ) Tetra- $n$-butylammonium salt of bromphthalein magenta $\mathrm{B}$; $(4 \mathrm{E}$ to $6 \mathrm{E})$ tetraethylammonium salt of bromphthalein magenta $\mathrm{E}$. Concentrations: (1) $10^{-4} M$; (2) $4 \times 10^{-5} M$; (3) $2 \times 10^{-5} M$; (4) $10^{-5} M$; (5) $5 \times 10^{-6} M$; 6) $2 \times 10^{-6} \mathrm{M}$.

On the other hand, the molar absorbancy near $400 \mathrm{~m} \mu$, the region in which bromphthalein magenta in the form of the free acid has a strong absorption band, shows a slight increase with dilution. The rate of change of the indexes with dilution is somewhat irregular.

In accounting for the change in the intensity of absorbancy with dilution, one possibility to consider is an equilibrium between ion pairs and quadrupoles, such as that which has been postulated to interpret conductance phenomena [65]. ${ }^{24}$

\footnotetext{
${ }^{24}$ As no Tyndall beam was detected, and no precipitate was visible in any of the flasks, even after 10 months, the salts may be assumed to have been molecularly dispersed in the benzene solution. Kraus and coworkers in studying the conductance of tetraalkylammonium salts in anisole, ethylene dibromide, and ethylene dichloride observed dilution effects which they attributed to sorption [58].
} 
It is questionable whether such an equilibrium would account for spectral changes of the kind observed, and also whether quadrupoles would exist in measurable quantity at such low concentrations of solute. A second possible explanation is found in evidence of the reaction of the salt with glass. The flasks that contained the solutions corresponding to curves $1 \mathrm{~B}$ and $4 \mathrm{E}$ were drained, washed three times with benzene, and allowed to drain until dry. Freshly boiled distilled water containing phenolphthalein added to each flask produced a pink color that was more vivid in the flask that had contained the ethyl (less soluble) derivative. ${ }^{25}$ A possible interpretation of this behavior is reaction of the tetraalkylammonium salt with $\mathrm{HO}-\mathrm{Si}(-\mathrm{O}-)_{3}$ at the glass surface, ${ }^{26}$ giving $\mathrm{R}_{4} \mathrm{~N}^{+}-\mathrm{OSi}(-\mathrm{O}-)_{3}$ with bromphthalein magenta as byproduct, followed by hydrolysis of the $\mathrm{R}_{4} \mathrm{~N}^{+}-\mathrm{O}-\mathrm{Si}$ portion of the complex upon the addition of water, to yield $\mathrm{R}_{4} \mathrm{~N}^{+}$, $\mathrm{OH}^{-}$, and $\mathrm{HO}-\mathrm{Si}(-\mathrm{O}-)_{3}$. The greater size of the hydrocarbon groups in the tetrabutylammonium salt not only explains its higher solubility in benzene, but also might well impede its reaction with glass.

In figure 15 the molar absorbancy curve for the tetraethylammonium salt of bromphthalein magenta $E$ in benzene (curve 1 ) is compared with those obtained for solutions in ethyl acetate (curve 2 ), ethylene dichloride (curve 3), and 95-percent ethyl alcohol (curve 4). Curve 4 shows two wellmarked absorption bands near 26,000 and 32,000 $\mathrm{cm}^{-1}$, in place of the three shallow bands in the ultraviolet noted previously for the solution in benzene (page 242). In curve 3, a shift of about $2,500 \mathrm{~cm}^{-1}(10 \mathrm{~m} \mu)$ towards the infrared is shown for the main absorption band. In other respects the molar absorbancy curves obtained for the tetraethylammonium salt in the four solvents are qualitatively similar. The only marked difference is in the intensity of the main absorption band, which is not significantly different for the solutions in ethylene dichloride and 95-percent alcohol but is lower for the solution in ethyl acetate and still lower for that in benzene. The molar absorbancy curves for $5 \times 10^{-5}-M$ solutions in ethylene dichloride and alcohol did not differ from those

\footnotetext{
${ }_{25}$ Negative tests were obtained with three flasks chosen at random from the stock. Other organic bases have been observed to undergo adsorption or reaction at the surface of glassware.

26 The oxygen atoms which are not attached to hydrogen are considered to be linked to silicon atoms in the glass network $[66,67]$.
}

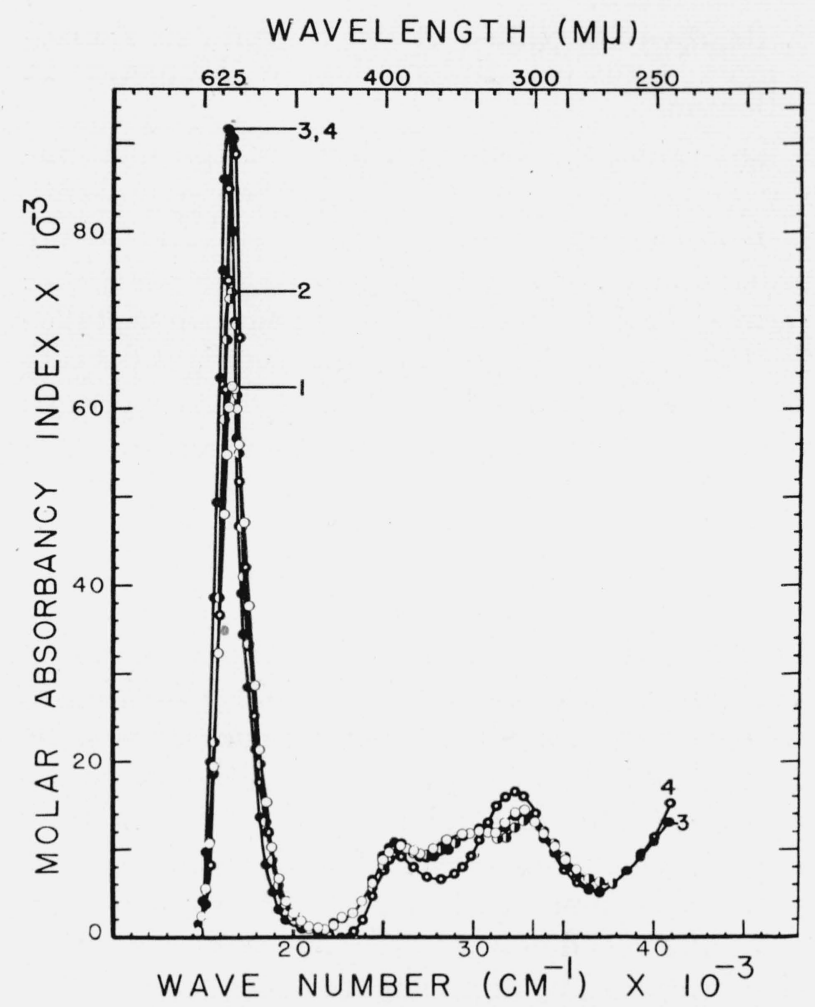

Figure 15. Molar absorbancy curves for tetraethylammonium salt of bromphthalein magenta $E$.

(1 to 3) $10^{-5} \mathrm{M}$ in 1-cm cell, in benzene, ethyl acetate, and ethylene dichloride, respectively; (4) $5 \times 10^{-5} \mathrm{M}, 2.5$-mm cell, in 95-percent ethyl alcohol.

obainted for $10^{-5}-M$ solutions. Furthermore, the same curve was obtained for the potassium salt of bromphthalein magenta $\mathrm{E}$ in alcohol as for the tetraethylammonium salt. Due to limited solubility, absorbancy curves for $5 \times 10^{-5}-M$ solutions of the tetraethylammonium salt in benzene and ethyl acetate could not be obtained. It can be noted, however, that at twice this concentration the molar absorbancy at $605 \mathrm{~m} \mu$ found for the tetrabutylammonium salt in benzene (1B, fig. 14) is less than that found for the tetraethylammonium salt in ethylene chloride and in alcohol. As there is doubt that the ethyl and butyl salts remained entirely in solution in benzene (see preceding paragraph), the low intensity of the main absorption band for the solution in benzene may, at least tentatively, be accounted for on that basis. A similar explanation serves for the data found for the ethyl acetate solution. The dielectric constant for ethyl acetate is approximately 4.5 , or about twice as great as that for benzene; this probably explains its somewhat higher solvent action upon the salts in question. 
6. Relative Stabilities of the Substituted Ammonium Salts of Bromphthalein Magenta in Benzene

The primary, secondary, tertiary, and quaternary ammonium salts of bromphthalein magenta in benzene appear to increase in stability in the order named. Complete quantitative data concerning the rate and extent of changes upon standing have not been obtained, because slight fluctuations in temperature are great enough in some cases to account for part of the observed changes. It is possible, however, to make several statements about the stability of the solutions.

In regard to solutions containing primary amines, it has already been stated that $5 \times 10^{-5}-M$ bromphthalein magenta in benzene with up to 100 molar equivalents of (a) cyclohexylamine, (b) $n$-heptylamine, or (c) n-octylamine showed no measurable change for at least 4 hours. In 3 weeks solution (a) had lost all its color. With only 1 molar equivalent of amine, the solution was stable for at least a day and retained some color at the end of 3 weeks. The same concentration of dye with 180 molar equivalents of $n$-butylamine showed noticeable fading in two hours-a 9-percent decrease in transmittancy at $540 \mathrm{~m} \mu$. When kept for several weeks all of the solutions showed fading, which was greater at the higher concentrations of base. The most concentrated solutions when almost colorless contained minute quantities of iridescent green crystals that gave a blue solution in alcohol.

A solution that contained 1.4 molar equivalent of piperidine showed changes after 2 days that appeared to involve both fading and reaction of the base with glass. There are two positions in the dye molecule where reaction with a primary or secondary amine might occur. One is the ester group, that might be saponified if the solution absorbed water. The other position is the central carbon, which can add $\mathrm{RNH}-$ or $\mathrm{R}_{2} \mathrm{~N}-$, with simultaneous addition of $\mathrm{H}-$ to the quinoid oxygen atom. This latter reaction is analogous to the conversion of triphenylmethane dyes to the carbinol form in aqueous solutions of relatively high $\mathrm{pH}$. An example is the fading of some of the sulfonephthalein indicators in alkaline media. A smaller tendency in this direction can be expected for primary amines that contain a bulky $\mathrm{R}$ group, and secondary amines should react less readily than primary amines. The amines that have been investigated behave in accordance with this prediction. Thus it happens that the amines showing the least stability with bromphthalein magenta in benzene are those most easily determined in water; that is, primary amines of low molecular weight. The bases with large alkyl groups are difficultly soluble in water, but give sufficiently stable solutions with bromphthalein magenta for measurement of their reactivity with this compound.

Tertiary amines cannot react with the central carbon atom in the manner discussed in the preceding paragraph. It is found experimentally that the transmittancy of solutions of bromphthalein magenta with tertiary amines or diarylguanidines changes by 1 or 2 percent at most in a month, and even after a year there is no fading evident to the eye.

As for the tetraalkylammonium salts of bromphthalein magenta in benzene, changes such as those discussed in section IV-5 were not evident for solutions that were not diluted. The tetraethylammonium salt gave the same transmittancy values after 9 days, and showed no visual change in over a year. Apparently the extent of change is limited by the area of the glass surface with which the solution comes in contact.

It might be pointed out that as the indicator alone and many of the amines alone give stable solutions in benzene, it has been possible to keep stock solutions of both indefinitely. The desired mixtures can then be prepared as needed.

\section{Relative Strengths of Some Organic Bases in Benzene}

In the preceding sections, several statements were made about the comparative strengths of organic bases as evidenced by their ionization constants in water and their reactivity with bromphthalein magenta in benzene. Some consideration was also given to the probable mechanism of the reaction of the bases with the indicator acid. Before discussing the reaction constants for organic bases, a few words should be said about the reactions from a purely empirical standpoint. In figure 16 the number of moles of base per mole of indicator acid has been plotted against the absorbancy at $540 \mathrm{~m} \mu^{27}$ for $5 \times 10^{-5}-M$ bromphthalein

\footnotetext{
27 Values for absorbancy, which is the negative logarithm of the transmittancy, can be read directly from the instrument but not so precisely as the values for transmittancy. The absorbancies given in figure 16 were computed from the transmittancies. Absorbancy is preferred to the equivalent term "optical density."
} 


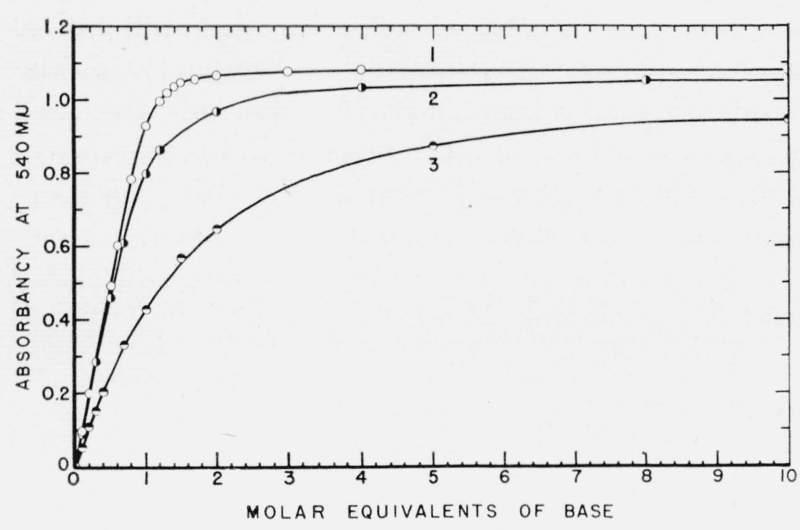

FiguRE 16. Change in absorbancy produced by adding different amounts of base, expressed in molar equivalents of the indicator, to $5 \times 10^{-5}-M$ bromphthalein magenta in benzene.

(1) Bromphthalein magenta E plus di-o-tolylguanidine; (2) bromphthalein magenta $\mathrm{E}$ plus diphenylguanidine; (3) bromphthalein magenta $\mathrm{B}$ plus triethylamine.

magenta in benzene with (1) di-o-tolylguanidine; (2) diphenylguanidine; and (3) triethylamine. The smoothness of the curves makes it possible to obtain by interpolation the absorbancy for other concentrations of base. In fact, a good approximation of the correct curve may be drawn from only a few experimental points. Equally smooth curves were obtained for the reaction of bromphthalein magenta with secondary amines. It is apparent from the figure that the reactivity of the three bases decreases in the order given. The reaction had reached completion with 10 molar equivalents of di-o-tolylguanidine. Slightly more than this quantity of diphenylguanidine was required, and about ten times as much triethylamine. In the reaction of any one of these bases with bromphthalein magenta, intermediate color tones can be detected visually with great ease. The possibility of application to rapid colorimetric analysis is therefore evident.

In section IV-4, a simple mechanism for the reaction of bromphthalein magenta with organic bases in benzene is proposed, namely, the addition of the indicator acid to the base in a $1: 1$ ratio. This may be an oversimplification, or may not be applicable in all cases. However, it serves as a starting point for numerical comparisons. The type of equation for the reaction was $\mathrm{B}+\mathrm{HOR} \rightleftarrows$ BHOF or, using the symbols $\mathrm{A}$ and $\mathrm{S}$ to represent the acid HOR, and the product, $\mathrm{B} \stackrel{+}{\mathrm{H}} \overline{\mathrm{O}} \mathrm{R}$, respec- tively, the equilibrium constant may be formulated as follows:

$$
[\mathrm{S}] /\{[\mathrm{B}] \times[\mathrm{A}]\}=K_{b} .
$$

From this, the following expression is obtained:

$$
\log \{[\mathrm{S}] /[\mathrm{A}]\}-\log [B]=\log K_{b} .
$$

When the value of $[\mathrm{S}] /[\mathrm{A}]$ is unity, $\log K_{b}=-\log$ [B]. In figure 17 experimental data for the reac-

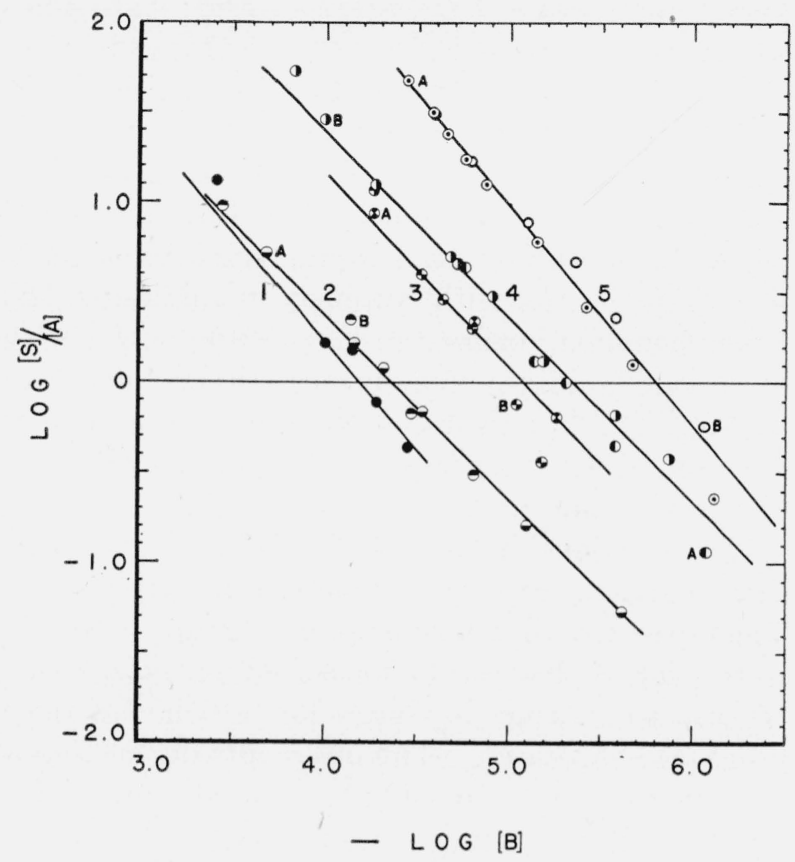

FIGURE 17. Graphical evaluation of equilibrium constants for the reaction of bromphthalein magenta with different bases in benzene.

(1) Bromphthalein magenta $\mathrm{E}, 1.6 \times 10^{-5} \mathrm{M}$, with di- $n$-butylamine; $(2 \mathrm{~A})$ $5 \times 10^{-5}-M$ bromphthalein magenta $\mathrm{B}$, and (2B) $4 \times 10^{-5}-M$ bromphthalein magenta $\mathrm{E}$, with triethylamine; ( $3 \mathrm{~A}$ and $3 \mathrm{~B}$ ) $5 \times 10^{-5-} \mathrm{M}$ bromphthalein magenta $\mathrm{E}$ with piperidine; (4A) $4 \times 10^{-5}-M$ bromphthalein magenta $\mathrm{E}$, and (4B) $5 \times 10^{-5}-M$ bromphthalein magenta $\mathrm{B}$, wish diphenylguanidine; (5A) $5 \times 10^{-5}-M$ bromphthalein magenta $\mathrm{E}$, and (5B) $2.5 \times 10^{-5}-M$ bromphthalein magenta $\mathrm{B}$, with di-o-tolylguanidine.

tion of bromphthalein magenta with five different bases are presented, with values for $\log \{[\mathrm{S}] /[\mathrm{A}]\}$ as ordinates and values for $-\log [\mathrm{B}]$ as abscissas. In order to calculate the values for [S], [A], and [B], data such as those used in plotting figure 16 were used. The ratio of the absorbancy for a given concentration of base to the absorbancy for complete conversion of the indicator acid to the salt gives the fraction of the indicator that was converted to the salt. This fraction multiplied by the molar concentration, $C_{a}$, of indicator originally in the solution gives [S], the concentration of the salt in moles per liter. The concentra- 
tion of residual_acid, [A], is $C_{a}-[\mathrm{S}]$. If $C_{b}$ is the molar concentration of base added to the mixture, the concentration of base in the equilibrium mixture, [B], equals $C_{b}-[\mathrm{S}]$. From a series of values for $[\mathrm{S}],[\mathrm{A}]$, and $[\mathrm{B}]$ calculated in this way for solutions containing the same initial concentration of the indicator acid with different amounts of base, the curves shown in figure 17 were constructed. ${ }^{28}$ These curves correspond to the following mixtures in benzene as solvent: (1) Bromphthalein magenta $\mathrm{E}, 1.6 \times 10^{-5} M$, with di- $n$ butylamine; (2A) bromphthalein magenta B, $5 \times 10^{-5} M$, with triethylamine; (2B) bromphthalein magenta $\mathrm{E}, 4 \times 10^{-5} \mathrm{M}$, with triethylamine; (3A and $3 \mathrm{~B}$ ) bromphthalein magenta $\mathrm{E}, 5 \times 10^{-5}$ $M$, with piperidine; (4A) bromphthalein magenta $\mathrm{E}, 4 \times 10^{-5} M$, with diphenylguanidine; (4B) bromphthalein magenta $\mathrm{E}, 5 \times 10^{-5} \quad M$, with diphenylguanidine; (5A) bromphthalein magenta $\mathrm{E}, 5 \times 10^{-5} \mathrm{M}$, with di-o-tolylguanidine; and (5B) bromphthalein magenta $\mathrm{B}, 2.5 \times 10^{-5} \mathrm{M}$, with di-o-tolylguanidine.

The relationship postulated in eq 2, if valid, should give linear curves, with unit negative slope. The lines were drawn so as to give greatest weight to the values that can be measured with the greatest precision, that is, those for which the ratio $[\mathrm{S}] /[\mathrm{A}]$ is near unity. The intercepts on the $x$-axis at zero value for $\log \{[\mathrm{S}] /[\mathrm{A}]\}$ give the values for $\log K_{b}$. These values, together with the values for the slope, are given in table 5 . It is apparent from figure 17 that if the lines had been drawn so as to give slightly different values for the slope, the values obtained for $\log K_{b}$ would have been practically unchanged. For triethylamine and diphenylguanidine, the slope of the line differs by only 3 percent from the theoretical value. The greatest deviation, 23 percent, occurs in the case of ditolylguanidine. Factors such as polymerization of the base may complicate the reaction. For example, Hunter and Marriott [68], from measurements of molecular weights in naphthalene, concluded that 1,3-diphenylguanidine and related compounds are partly associated in this solvent. However, the solutions used in the experiments described in this paper were so dilute that the degree of association of the base should be very small. The authors are inclined to attribute

${ }_{28}$ To be exact, the values used in constructing the curves were the averages from two sets of measurements-one for the wave length 400 or $405 \mathrm{~m} \mu$ and the other for the wavelength 540 or $580 \mathrm{~m} \mu$. discrepancies largely to experimental difficulties, such as minor fluctuations in temperature, the tendency of the base to react or become absorbed at a glass surface, and the unavailability of adsorption cells of suitable length at the time the spectrophotometric measurements were made.

TABLE 5.-Values of $\log K_{b}, K_{b}$, and slope for reaction of organic bases with bromphthalein magenta in benzene

\begin{tabular}{l|r|r|r}
\hline \hline \multicolumn{1}{c|}{ Organic base } & $\log K_{b}$ & \multicolumn{1}{c|}{$K_{b}$} & $\begin{array}{l}\text { Slope of } \\
\text { curve }\end{array}$ \\
\hline & & & \\
Di- $n$-butylamine $\ldots .19$ & $1.55 \times 10^{4}$ & -1.17 \\
Triethylamine & 4.36 & $2.3 \times 10^{4}$ & -1.03 \\
Piperidine & 5.08 & $1.2 \times 10^{5}$ & -1.08 \\
Diphenylguanidine & 5.35 & $2.2 \times 10^{5}$ & -1.03 \\
Ditolylguanidine & 5.80 & $6.3 \times 10^{5}$ & -1.23 \\
\hline
\end{tabular}

In figure 18 the numerical values of $\log K_{b}$ in benzene for the five bases are plotted as ordinates, with the values of $\mathrm{p} K_{b}$ in water as abscissas. It will be remembered that $\mathrm{p} K_{b}$ is the reciprocal of the logarithm (negative logarithm), of the ionization constant of the base in water (see section $I V-4)$. From the figure it is evident that the relationship between $\log K_{b}$ in benzene and $\mathrm{p} K_{b}$ in water is remarkably constant for triethylamine and the two arylguanidines. Whether a consistent relationship holds for other tertiary amines and secondary amines can be determined only by further studies. We hope to extend these studies, under improved experimental conditions.

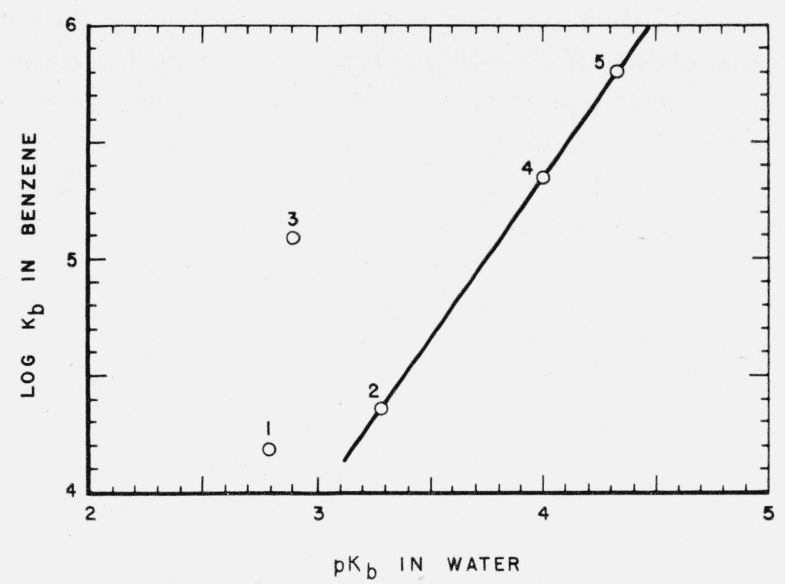

Figure 18. Comparison of $\log K_{b}$ in benzene with $\mathrm{p} K_{b}$ in water.

$K_{b}=$ equilibrium constant for reaction of base with bromphthalein magenta; $\mathrm{p} K_{b}=\log$ reciprocal of ionization constant of the base in water. (1) Di- $n$ butylamine; (2) triethylamine; (3) piperidine; (4) 1,3-diphenylguanidine; (5) 1,3-di-o-tolylguanidine. 


\section{Explanation for the Different Color Reactions of Bromphthalein Magenta With Organic Bases in Benzene}

As described earlier in this paper, the color change accompanying the reaction of an organic base with bromphthalein magenta in benzene is different for primary, secondary, and tertiary amines. Moreover, the quaternary ammonium salts, while resembling most nearly the product with secondary amines, show characteristic differences from the partially substituted ammonium salts of the indicator.

The conductance of quaternary ammonium salts in benzene and other solvents of low dielectric constant has been studied extensively, particularly by Walden and Kraus and their coworkers $[28,57 \mathrm{~b}]$. These studies have given strong experimental support to Bjerrum's theory of ionpairs [69]. In harmony with the views of these authors, the tetraethylammonium and tetra- $n$ butylammonium salts of bromphthalein magenta may be considered to exist in dilute benzene solution predominantly as individual ion-pairs, $\mathrm{M}^{+} \mathrm{A}^{-}$, $\mathrm{M}^{+}$representing the cation, and $\mathrm{A}^{-}$the anion. To a minor degree, dissociation of $\mathrm{M}^{+} \mathrm{A}^{-}$into $\mathrm{M}^{+}$ and $\mathrm{A}^{-}$, or association into triple-ions, $\mathrm{MAM}^{+}$ and $\mathrm{AMA}^{-}$, or into still more complex groupings may occur. According to Bjerrum, the extent of dissociation or association will not be evident in the optical behavior of the salt.

The difference in the optical behavior of the quaternary ammonium (blue) and tertiary ammonium (magenta) salts of bromphthalein magenta in benzene is paralleled by differences in conductances found for salts of the two classes $[28,57]$. In the tertiary ammonium salts, the existence of a proton in place of one of the alkyl groups of the quaternary ammonium salt permits a closer approach of the positive and negative ions to each other, with a consequent increase in the Coulombic force. However, the change in behavior that accompanies this modification in structure is undoubtedly due in part to the formation of a hydrogen bridge between the nitrogen of the base and the oxygen of the indicator acid. ${ }^{29}$ The structure of the salt can be indicated in a general way by the formula $\mathrm{B}-\mathrm{H}^{+}$. . $\mathrm{O} \mathrm{R}$.

In accounting for the purple-blue color of benzene solutions of the secondary ammonium salts

\footnotetext{
${ }^{29}$ See discussion in the introduction and in section $\mathrm{IV}-4$.
}

of bromphthalein magenta, other observations are of value. During titrations of secondary amines in benzene with bromphthalein magenta as indicator, it was discovered that the initial effect of adding an acid to the purple-blue solution was to produce a magenta color, which was changed to yellow by an excess of acid. When an excess of base was then added, the magenta color reappeared, but the purple-blue color could not be restored by any quantity of base. ${ }^{30}$ Further tests showed that the conversion of the purple-blue color to magenta was not characteristic of acids but was produced by a variety of oxygen compounds, such as dioxane, acetone, ethyl acetate, and alcohols. ${ }^{31}$ The magenta color is very similar to that of mixtures of bromphthalein magenta with tertiary amines in benzene. Spectrophotometric measurements of solutions that contained $5 \times$ $10^{-5}-M$ bromphthalein magenta $\mathrm{E}$ and $5 \times 10^{-4}-M$ piperidine in benzene, with from 4 to 6 percent of absolute ethyl alcohol by volume, showed that the absorbancy curves of these solutions are very similar to, though not identical with, the characteristic limiting curve for the reaction of a tertiary amine with bromphthalein magenta in benzene. $^{32}$

It can hardly be questioned that the change in color produced by dioxane, acetone, and other oxygen compounds arises from their interaction with the amine. Numerous studies of hydrogen bridges between amines and other types of compounds have been made by infrared and Raman spectroscopy, solubility measurements, and other methods. From these studies, the ability of amines to form hydrogen bridges with compounds containing active hydrogen, such as alcohols and haloforms, appears to be well established. Associated molecules in which the hydrogen of the amine is bridged to the oxygen of another molecule seem to be less common. However, Gordy [70] obtained spectroscopic evidence for association of this type in mixtures of aniline with acetone,

\footnotetext{
${ }^{30}$ It was noted subsequently that a similar behavior has been described for bromphenol blue, the sulfonephthalein analog of bromphthalein magenta [41].

31 Normal mercaptans with $8,10,12$, or 14 carbon atoms were without effect. For additional compounds that produce the effect, see section IV-10.

32 Continued addition of alcohol produced a gradual shift to the blue color and molar absorbancy curve characteristic of the tetraethylammonium or potassium salts of bromphthalein magenta in 95-percent alcohol. In the absence of piperidine, the effect of 4 to 6 percent of alcohol on the absorbancy curve of the indicator was almost negligible. The molar absorbancy curve for $5 \times 10^{-6}-M$ bromphthalein magenta in absolute ethyl alcohol is the same as that found for the potassium and tetraethylammonium salts in 95-percent alcohol, but in more concentrated solutions the presence of unchanged indicator is evident.
} 
esters, and ethers. Furthermore, according to Huggins [15], ammonium hydrogens can be expected to form stronger bridges to oxygen compounds than amino hydrogens. Consequently, formula II in figure 19 provides a reasonable structure for the magenta complex formed in

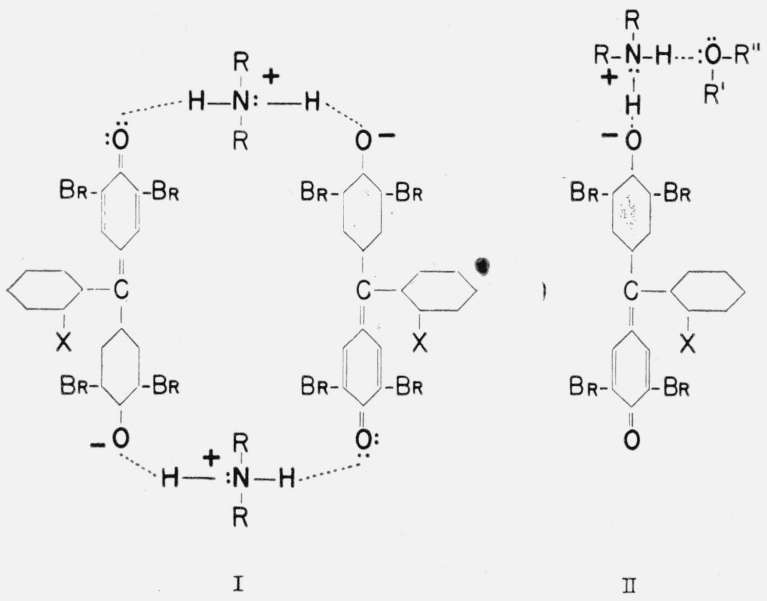

Figure 19. Formulas postulated for dialkylammonium salts of bromphthalein magenta.

I, Dimer formed in benzene and other inert solvents; II, monomer formed in presence of an oxygen compound. $R$ and $R^{\prime}=$ alkyl groups; $R^{\prime \prime}=$ hydrogen, alkyl, or other organic radical.

benzene solutions of bromphthalein magenta, a secondary amine, and an oxygen compound, $\mathrm{R}^{\prime} \mathrm{OR}^{\prime \prime}$. When the purple-blue salt is formed by reaction of bromphthalein magenta with \& secondary amine in benzene, it is logical to assume that in the absence of an oxygen compound the hydrogen atom linked to oxygen in formula II is involved in some other type of bridge. A structure for the purple-blue salt is suggested in figure 19, formula I. That is, a dimer, stabilized by resonance, is postulated. This hypothesis is consistent with the fact that the color of the secondary ammonium salt of bromphthalein magenta is intermediate between that of the tertiary ammonium salt and the quaternary ammonium salt. Additional support is furnished by studies of the molar polarization of partially substituted ammonium picrates in benzene as a function of concentration, performed in this laboratory by A. A. Maryott. Tertiary ammonium picrates exhibit no association, while secondary ammonium picrates are associated appreciably in a way that results in some cancellation of the dipole moments of the individual molecules of the salt. Primary ammonium picrates are still more highly associated.
When dioxane is used as solvent, there is no evidence of association for any one of the three types of salt [71].

As for the primary ammonium salts of bromphthalein magenta, which show optical resemblances to both the secondary ammonium and the tertiary ammonium salts, the following explanation is suggested. A dimer may form, as in the case of the secondary ammonium salt, but the third hydrogen of the ammonium ion can participate in bridge formation. As a result, the other nitrogen bridges are weakened.

It seems quite likely that phenomena of the kind so readily observed in the reaction of bromphthalein magenta with bases in benzene play an important role in numerous other acid-base reactions.

\section{Reaction of Bromphthalein Magenta in Benzene With Miscellaneous Other Organic Bases}

Ammonia.-A blue color results when dry ammonia is passed into bromphthalein magenta in benzene, and there is spectrophotometric evidence of an absorption band near $575 \mathrm{~m} \mu$. However, the product separates so rapidly from solution that quantitative studies are not possible.

Alkanolamines and other oxygen-containing amines.-As described in section $\mathrm{IV}-8$, addition of an alcohol or other oxygen compound to the blue solution of bromphthalein magenta and a secondary amine in benzene changes the color to magenta. It is not surprising, therefore, that oxygen within the secondary amine molecule, as in morpholine or methylmonoethanolamine, $\mathrm{CH}_{3} \mathrm{NHCH}_{2} \mathrm{CH}_{2} \mathrm{OH}$, produces a similar effect. For example, a small amount of morpholine with bromphthalein magenta in benzene produces a blue color, which changes to magenta with continued addition of morpholine. A trace of methylmonoethanolamine produces a blue color slightly tinged with red, and a larger quantity gives a magenta color. Such color phenomena, which at first seemed puzzling, can readily be understood and predicted by applying the explanation offered in section IV-8. That is, when relatively few molecules of the oxygenated amine are present their amino groups react with the indicator acid to give the blue dimer; when surplus molecules are present their oxygen groups become linked through hydrogen with the nitrogen atoms in the dimer, and the dimer changes to the magenta-colored monomeric 
salt. As the second reaction may occur before the first change is complete, there is likely to be evidence of the magenta color, together with the blue. The "normal" behavior of primary amines is likewise affected by oxygen groups within the molecule. Triethanolamine and $N$-methyl- or $N$-ethylmorpholine, on the other hand, give the magenta color produced by any tertiary aliphatic amine. The change in the color of bromphthalein magenta caused by any particular amine depends upon the character of its nitrogen atom (primary, secondary, or tertiary), upon the nature and number of its oxygen groups, and upon the concentrations of the amine and the indicator acid. The behavior of concentrated solutions in general is complicated by overlapping effects. Sensitive color reactions, such as those of bromphthalein magenta with simple amines in benzene, are helpful in gaining an understanding of more complex systems.

Alkaloids.-Alkaloids and other drugs of basicity comparable to that of aliphatic amines should show color reactions with bromphthalein magenta like those already described, provided steric or other factors do not interfere. A few tests with alkaloids containing tertiary amino groups gave results of the character anticipated. For example, a minute quantity of brucine, codeine, nicotine, quinine, or strychnine produced a magenta tone with bromphthalein magenta in benzene. Brucine, codeine, and nicotine were added as the free bases. The other two alkaloids, available as the sulfate, were dissolved in water, a trace of alkali was added, and the base was extracted with benzene. The benzene extract gave an immediate color reaction with the indicator.

One may predict the behavior of other alkaloids and drugs. For instance, coniine ( $\alpha$ - $n$-propylpiperidine) should give a blue color with bromphthalein magenta in benzene, but the majority of the alkaloids are likely to give a magenta tone.

Aromatic amines.-Aniline, dimethylaniline, and similar aromatic bases are too weakly basic to show measurable reaction with bromphthalein magenta at concentrations such as those dealt with in the preceding sections of this paper. In amounts greater than about 100 molar equivalents, aniline or dimethylaniline produce a perceptible change of color from yellow to amber. With increasing amounts of the aromatic amine, the color gradually deepens. Spectrophotometric measurements showed that no stoichiometric reaction occurs.

Tetrahydroquinoline and decahydroquinoline.Decahydroquinoline, even in a very small quantity, gives a blue color with bromphthalein magenta in benzene, showing the usual behavior of a secondary amine. The basicity of tetrahydroquinoline, on the other hand, is weakened by the partial aromatic character of the molecule. In optical behavior, it shows some similarity to primary aliphatic amines. Absorbancy curves for different mixtures of bromphthalein magenta $\mathrm{E}$ with tetrahydroquinoline in benzene are shown in figure 20. These curves correspond to $10^{-4}-M$

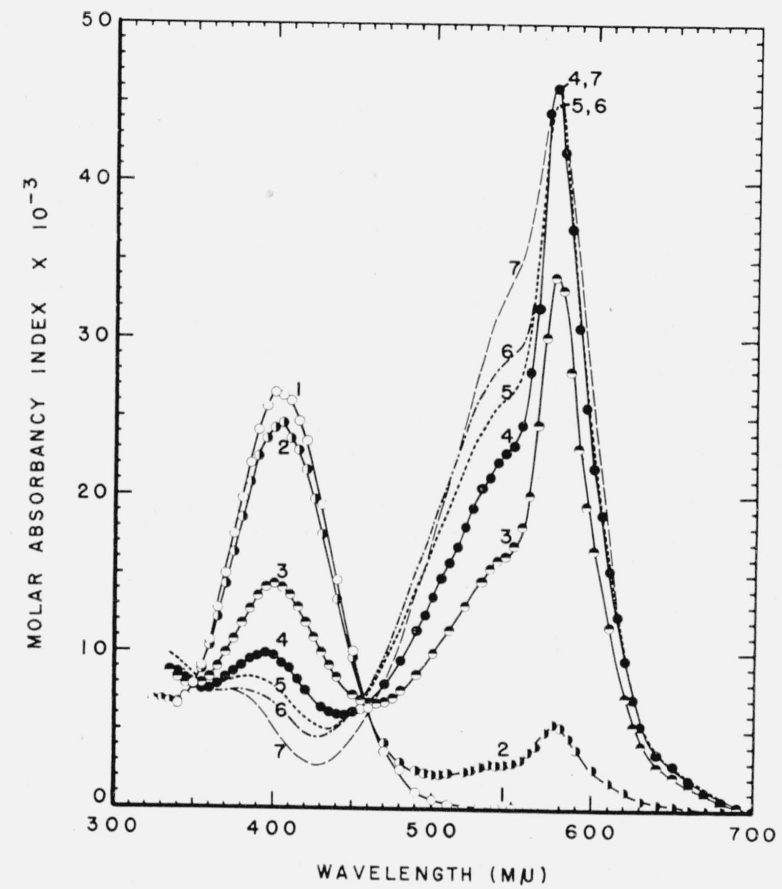

Figure 20. Absorbancy curves for bromphthalein magenta $\mathrm{E}, 10^{-4}-\mathrm{M}$ in benzene, 2.5- $\mathrm{mm}$ cell, with various concentrations of tetrahydroquinoline expressed as molar equivalents of the indicator.

(1) None; (2) 80; (3) 500; (4) 800; (5) 1,200; (6) 1,680; (7) 10,000.

dye in a 2.5-mm cell, with the following concentrations of tetrahydroquinoline, expressed as molar equivalents of the indicator: (1) None; (2) 80; (3) 500 ; (4) 800 ; (5) 1,200 ; (6) 1,680 ; (7) 10,000 . In addition to the absorption band at $575 \mathrm{~m} \mu$, there is evidence of a second band near $540 \mathrm{~m} \mu$. For concentrations of tetrahydroquinoline up to 800 molar equivalents (curve 4), there are two fairly sharp isosbestic points, one near $350 \mathrm{~m} \mu$ and the other near $455 \mathrm{~m} \mu$. The absorption band 
near $575 \mathrm{~m} \mu$ reached a maximum index of 45,000 to 46,000 for about 800 molar equivalents of tetrahydroquinoline. When larger quantities of the base were added to the dye, the molar absorbancy index at $575 \mathrm{~m} \mu$ remained fairly constant, but the absorbancy near $540 \mathrm{~m} \mu$ increased, and the solution exhibited instability. Consequently, it was not possible to determine the limiting curve for the reaction. The lack of constancy of the molar absorbancy index near $575 \mathrm{~m} \mu$ may be due to incipient precipitation of the salt. Tetrahydroquinoline was present in the comparison cell when the data for curves 2,3 , and 4 were obtained. Insufficient tetrahydroquinoline was available to permit its use in the comparison cell during the measurements for curves 5, 6, and 7. Furthermore, the two sets of curves were obtained with two different samples which contained different amounts of a brown impurity. The lack of constancy of the isosbestic point near $455 \mathrm{~m} \mu$ in the case of curves 5,6 , and 7 is probably caused partly by the colored impurity and partly by incipient precipitation of the salt.

Alkyl-substituted guanidines.-This interesting class of nitrogen bases has been ignored by most investigators. A valuable contribution was made by Davis and Elderfield, who studied the effect upon the basicity of guanidine and its derivatives of variations in the kind of substituent and the position of substitution [72]. They pointed out that guanidine and its monoalkyl, $N, N$-dialkyl, and $N, N^{\prime}, N^{\prime \prime}$-trialkyl derivatives give aqueous solutions comparable in basic strength with aqueous potassium hydroxide. On the other hand, monophenylguanidine is considerably less basic than monoalkylguanidines and $N, N^{\prime}$ dialkylguanidines are weaker still. Pauling has suggested that resonance in the guanidinium ion may explain the high basicity of guanidine and the effect of substituents [17]. The study of acidbase reactions in organic media has been hampered by the lack of an organic base sufficiently strong to react with acetic, benzoic, and other weak organic acids. The data presented in section IV-7, indicate that $N, N^{\prime}$-diphenyl- and di-otolylguanidine are more strongly basic in benzene than piperidine, which is generally considered to be the strongest of the aliphatic amines. $N, N^{\prime}$ Dialkylguanidines should be more basic than the corresponding diarylguanidines, and $N, N^{\prime}, N^{\prime \prime}$ trialkylguanidines, still stronger. Unfortunately, such compounds are not available commercially at the present time. However, interest in guanidine derivatives is growing, and it is hoped that eventually a series of symmetrical di- and trialkyl derivatives will be obtainable. One would expect that $N, N^{\prime}, N^{\prime \prime}$-trialkylguanidines in which the alkyl groups contain 4 or more carbon atoms would possess sufficiently great basicity and solubility in inert solvents to prove useful in titrations of weak organic acids.

Experimental samples of $N, N^{\prime}$-dicyclohexylguanidine hydrochloride, monododecylguanidine, and the salts of a few other monosubstituted guanidine derivatives were obtained from a commercial source. ${ }^{33}$ Dicyclohexylguanidine hydrochloride was converted into the free base. As anticipated, this is more basic than diphenyl- or di-o-tolylguanidine. It also differs in giving a blue color instead of magenta when mixed with bromphthalein magenta in benzene. The usefulness of dicyclohexylguanidine is limited by its rather low solubility, which is little more than 0.0025 mole per liter of benzene. Monododecylguanidine also turns bromphthalein magenta blue and is difficultly soluble in benzene.

In other experiments, a suspension of monophenyl-, $\alpha$-naphthyl-, or $p$-tolylguanidine carbonate in distilled water was shaken for a few moments with benzene. Partial hydrolysis of the salt occurred, and a sufficient quantity of the free guanidine derivative dissolved in the benzene layer to give a blue color upon the addition of bromphthalein magenta. Commercial methylguanidine sulfate behaved similarly when a trace of alkali was added to its aqueous solution and the latter was then shaken with benzene.

In figure 21 are shown the molar absorbancy curve for bromphthalein magenta in benzene (curve A) and the limiting curves for its reaction with the following organic bases: (1) Cyclohexylamine; (2) diethylamine; (3) triethylamine; (4) tetra- $n$-butylammonium hydroxide; (5) 1,3-dicyclohexylguanidine; (6) tetrahydroquinoline. ${ }^{34}$ Curves 2, 3, and 4 show single maxima in the visible region. These occur at the following respective wave numbers $\left(\mathrm{cm}^{-1} \times 10^{-3}\right): 17.4$, $18.4,16.5$. For curve $\mathrm{A}$, the maximum in the

\footnotetext{
${ }_{33}$ The authors are indebted to the American Cyanamid Co. for these samples.

${ }_{34}$ The maximum molar absorbancy index for curve 4 is probably too low (see section $\mathrm{IV}-5$ ). Curve 6 was calculated from the data shown as absorbancy curve 4 in fig. 20 .
} 


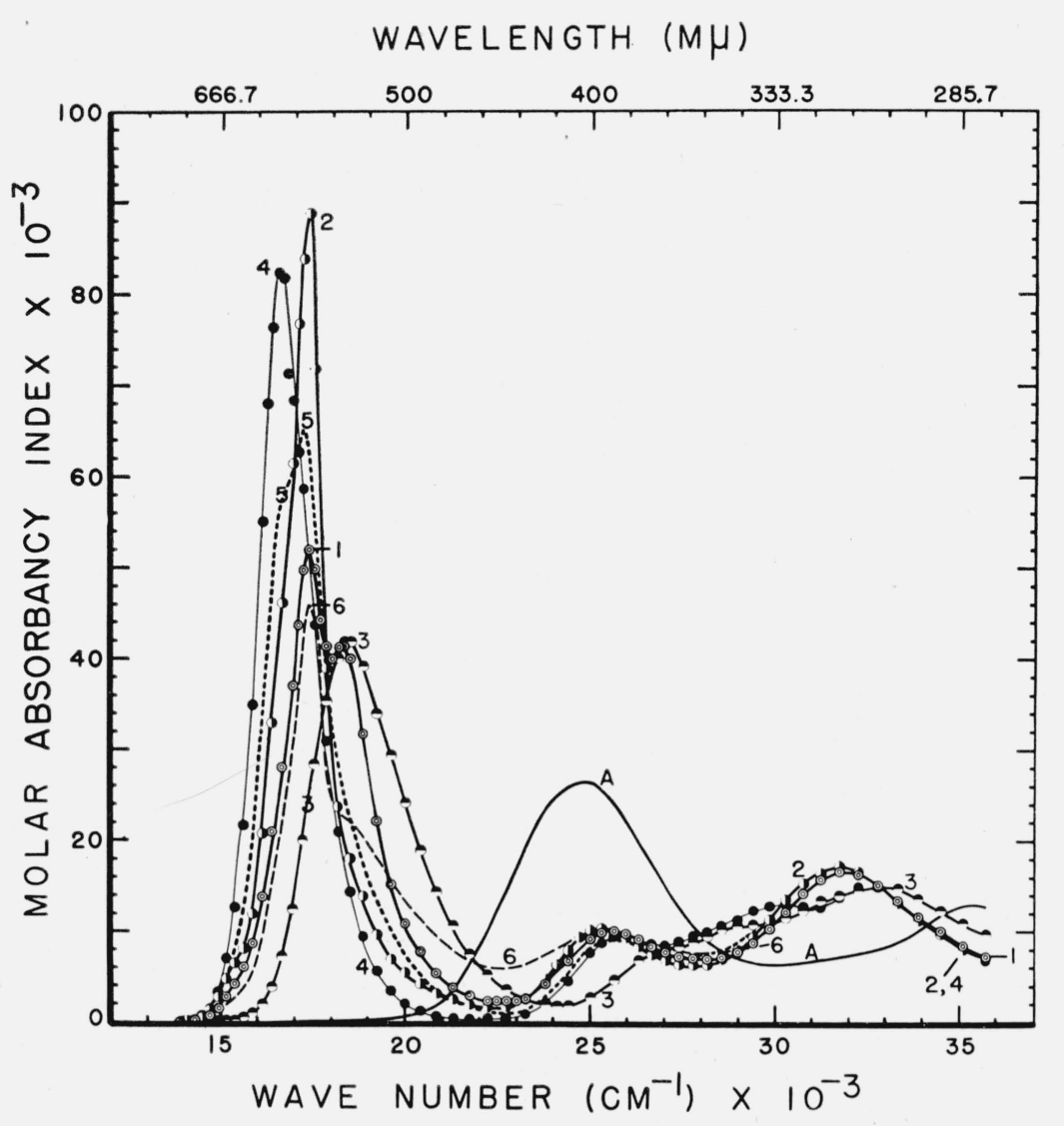

FIGURE 21. Molar absorbancy curves for bromphthalein magenta and its salts with different organic bases in benzene.

(A) Bromphthalein magenta E; (1) bromphthalein magenta E plus cyclohexylamine; (2) bromphthalein magenta E plus diethylamine; (3) bromphthalein magenta B plus triethylamine; (4) tetra- $n$-butylammonium salt of bromphthalein magenta $\mathrm{B}, 10^{-4} M$; $(5)$ bromphthalein magenta $\mathrm{E}$ plus $1,3-$ dicyclohexylguanidine; (6) bromphthalein magenta $\mathrm{E}, 10^{-4} \mathrm{M}$, plus 800 molar equivalents of tetrahydroquinoline.

visible region is located practically midway between the wave numbers 25.0 and $24.7 \mathrm{~cm}^{-1} \times$ $10^{-3}$. In the other curves, there is evidence of two maxima in the visible spectrum, as follows: for curves 1 and 6 , at 17.4 and 18.4 ; for curve 6 , at 16.5 and 17.4. An explanation has been suggested (section IV-8) for the behavior of the primary, secondary, tertiary, and quaternary alkylammonium compounds (curves 1 to 4). The behavior of tetrahydroquinoline (curve 6) indicates that its salt with bromphthalein magenta is partly dimerized and partly in the monomeric form, while the curve for dicyclohexylguanidine suggests that its salt may exist partly as a dimer and partly as ion-pairs. However, because the dicyclohexylguanidine was only 98 to 99 percent pure, further experimentation is necessary before this interpretation can be accepted.

Trimethylamine oxide.-Although this paper is concerned primarily with bases in which the nitro- gen atom acts as proton acceptor, it is of interest to compare the behavior of a tertiary amine oxide toward bromphthalein magenta in benzene with that of the tertiary amines, already discussed. Nylén found six amine oxides, including trimethylamine oxide, to be weaker bases than the corresponding amines in aqueous solution [73]. Some other published data for trimethylamine and trimethylamine oxide are summarized in table 6 . Obviously the addition of an oxygen atom to the amine nitrogen changes appreciably the character of the base. Not only is the polar character of the molecule greatly enhanced and the carbon to nitrogen bond increased in length, but the picrate is much less dissociated in nitrobenzene. Consequently, one would predict stable salt formation from the reaction of trimethylamine oxide and bromphthalein magenta. The hygroscopicity of trimethylamine oxide is so great that preparation of a benzene solution of known concentration was 
not attempted. However, some distinctive characteristics of the amine oxide could be detected.

TABLE 6.-Comparison of trimethylamine and trimethylamine oxide

\begin{tabular}{|c|c|c|}
\hline Physical property & $\begin{array}{l}\text { Trimethyl- } \\
\text { amine }\end{array}$ & $\begin{array}{l}\text { Trimethyl- } \\
\text { amine oxide }\end{array}$ \\
\hline Dipole moment $(D)$ & s 0.65 & b 5.02 \\
\hline Length of $\mathrm{C}-\mathrm{N}$ bond $(A)$ & c $1.47 \pm 0.02$ & d $1.54 \pm 0.02$ \\
\hline Dissociation constant of picrate in nitrobenzene $e$ & $1.46 \times 10^{-4}$ & 1. $73 \times 10^{-5}$ \\
\hline
\end{tabular}

a L. G. Groves and S. Sugden, J. Chem. Soc. (London) 1779 (1937); gas.

b E. P. Linton, J. Am. Chem. Soc. 62, 1945 (1940); in benzene at $45^{\circ}$ C.

c I. O. Brockway and H. O. Jenkins, J. Am. Chem. Soc. 58, 2036 (1936).

d M. W. Lister and L. E. Sutton, Trans. Faraday Soc. 35, 495 (1939).

e C. A. Kraus, J. Phys. Chem. 43, 231 (1939).

Trimethylamine was oxidized to trimethylamine oxide by 3-percent hydrogen peroxide, according to the method of Dunstan and Goulding [74], and crystallized as the dihydrate. The anhydrous product was obtained as a sublimate by Meisenheimer's procedure, namely, heating of the dihydrate in vacuo over phosphorus pentoxide [75]. Part of the sublimate was transferred quickly to a volumetric flask containing $5 \times 10^{-5}-M$ bromphthalein magenta in benzene. Although the amine oxide did not dissolve completely, the solution immediately assumed a magenta tone. The absorbancy curve for this solution (curve 2) is shown in figure 22, together with the curves for

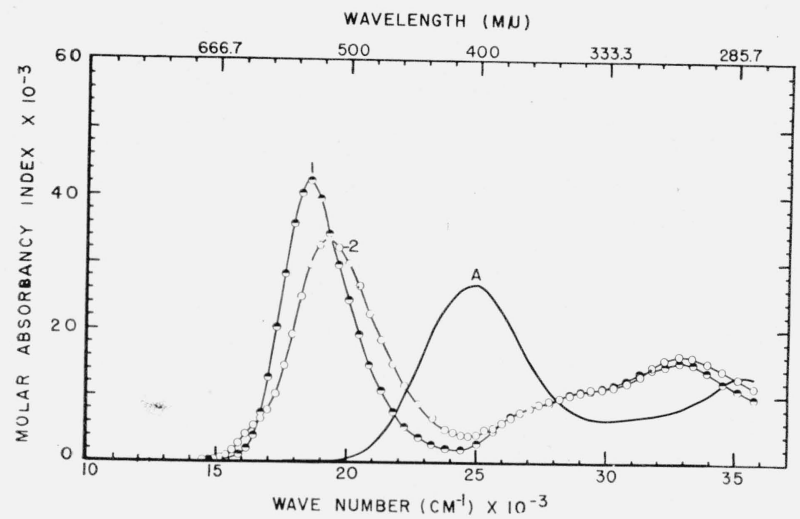

FIGURE 22. Molar absorbancy curves for bromphthalein magenta and its salts with different organic bases in benzene.

(A) Bromphthalein magenta $\mathrm{E}$; (1) bromphthalein magenta $\mathrm{E}$ plus triethylamine; (2) bromphthalein magenta $\mathrm{E}$ plus trimethylamine oxide.

bromphthalein magenta (curve A) and its triethylammonium salt (curve 1). The solution was probably saturated with trimethylamine oxide, since the absorption spectrum did not change after further shaking and the lapse of $2 \frac{1}{2}$ days. As compared with the triethylammonium salt, the absorbancy curve for the salt of trimethylamine oxide is shifted approximately $20 \mathrm{~m} \mu\left(700 \mathrm{~cm}^{-1}\right)$ toward shorter wavelengths, and the molar absorbancy index is 33,000 instead of 42,000 . In the region 310 to $380 \mathrm{~m} \mu\left(31,700\right.$ to $\left.26,300 \mathrm{~cm}^{-1}\right)$ the absorbancy curves for the triethylammonium and trimethylamine oxide salts are nearly identical. Curve 2 intersects curve $\mathrm{A}$ at the approximate wavelength $450 \mathrm{~m} \mu\left(22,220 \mathrm{~cm}^{-1}\right)$, while curve 1 intersects curve A near $460 \mathrm{~m} \mu\left(21,740 \mathrm{~cm}^{-1}\right)$. Degeneracy of the resonance structures of the anion of bromphthalein magenta appears to be less complete in the salt formed by combination of the indicator with trimethylamine oxide than in the salts formed by its combination with trialkylamines, indicating that the proton of the indicator acid undergoes a smaller displacement toward the oxygen of trimethylamine oxide than toward the nitrogen of triethylamine. This interpretation is in harmony with the low value reported by Kraus (table 6 , footnote e) for the dissociation into ions of the picrate of trimethylamine oxide in nitrobenzene.

A different sample of dehydrated trimethylamine oxide absorbed atmospheric moisture so quickly that the melting point corresponded to the dihydrate. Consequently, it is not certain that the sample that was mixed with bromphthalein magenta in benzene was free of water. No visual change was evident in the solution after several months. A striking change occurred when methyl or ethyl alcohol was added. One drop of the alcohol caused a marked fading of the magenta color, and several drops destroyed it completely, leaving the yellow color of the indicator acid. $n$-Butyl alcohol and cyclohexanol produced a smaller effect, and isopropyl alcohol was still less effective. Water was without visual effect, as were acetone, ethyl acetate, and dioxane. Pending further experimental work, it cannot be said whether the effect was due to the alcohol itself or to a trace of an acidic impurity. This behavior of the salt of trimethylamine oxide with bromphthalein magenta makes it readily distinguishable from solutions that contain the salt of bromphthalein magenta with unoxidized tertiary amines, such as trimethyl- or triethylamine. 
10. Reaction of Bromphthalein Magenta with Organic Bases in Other Organic Solvents. Discussion of Solvent Effects

Cyclohexane.-In this solvent, as in benzene, bromphthalein magenta gives different colors with primary, secondary, and tertiary amines. However, the salts formed have limited solubilities, as shown by the fact that a Tyndall beam could be observed when more than a minute quantity of the amine was present. Transmittancy curves for bromphthalein magenta $\mathrm{B}, 5 \times 10^{-5} \mathrm{M}$ in cyclohexane (curve 1), and for its products with diethylamine (curve 8) and with triethylamine (curves 2 through 7 ) are shown in figure 23. A

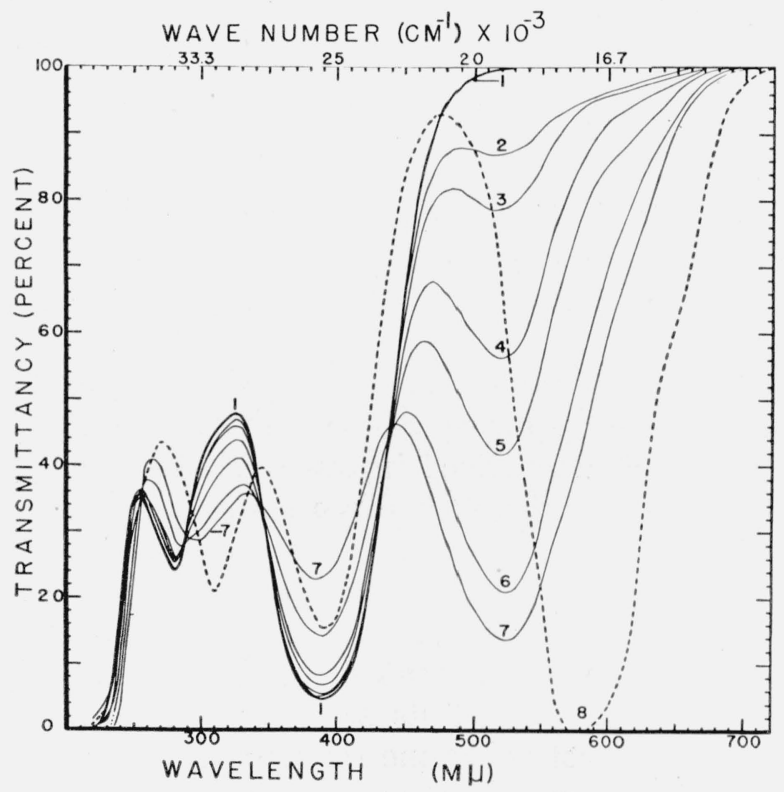

Figure 23. (1 to 7) Transmittancy curves for bromphthalein magenta $B, 5 \times 10^{-5} \mathrm{M}$ in cyclohexane, in 1-cm cell, with various concentrations of triethylamine expressed as molar equivalents of the indicator.

(1) None; (2) 1 ; (3) 2 ; (4) 5 ; (5) 8 ; (6) 20 ; (7) 40; (8) transmittancy curve for same solution of the indicator with 1 molar equivalent of diethylamine, $1-\mathrm{cm}$ cell.

1-cm absorption cell was used throughout. Curve 8 was obtained for the indicator after the addition of one molar equivalent of diethylamine. Curves 2 through 7 were obtained for solutions that contained the following successive concentrations of triethylamine, expressed as molar equivalents of the indicator acid: (2) 1 ; (3) 2 ; (4) 5 ; (5) 8 ; (6) 20 ; (7) 40. A Tyndall beam was not detected for the solutions that gave curves 2,3 , and 4 . With 20 molar equivalents of triethylamine, a Tyndall beam appeared after the solution had stood overnight, and with 40 molar equivalents, immediately after preparation of the solution. Maximum absorbancy in the visible region occurs 15 to $20 \mathrm{~m} \mu$ nearer the ultraviolet in cyclohexane than in benzene. The transmittancy curves show spreading at the higher wavelengths, probably due to scattering of light by suspended particles.

Ethylene dichloride.-In figure 24 are shown transmittancy curves for bromphthalein magenta $\mathrm{E}, 5 \times 10^{-5} \mathrm{M}$ in ethylene dichloride (curve 1), and for its reaction products with one molar equivalent of the following amines: (2) $n$-Butylamine; (3) di- $n$-butylamine; (4) triethylamine; (5) 1,3-di-otolylguanidine. Curve 6 was obtained for the $5 \times 10^{-5}-M$ solution of the tetraethylammonium salt of bromphthalein magenta $E$ in ethylene dichloride. A 5-mm absorption cell was used throughout.

The behavior of the tetraethylammonium salt in different solvents, including ethylene dichloride, was discussed above (sections $I V-5$ and $I V-8$ ), and it was concluded that the color of the anion shows no significant interaction of the anion with the cation and no appreciable solvent effect. The situation is different for the incompletely alkylated ammonium salts. As in benzene, these salts behave differently from the tetraalkylammonium salts. However, in ethylene dichloride primary, secondary, and tertiary ammonium salts show similar behavior instead of the characteristic differences observed for solutions in benzene. From figure 24 it is evident that in ethylene dichloride the $n$-butylammonium, di- $n$-butylammonium, triethyl-ammonium, and 1,3-di-o-tolylguanidinium salts of bromphthalein magenta possess similar transmittancy curves, with a maximum absorbancy (minimum transmittancy) near $570 \mathrm{~m} \mu$. This "leveling action" of ethylene dichloride may be explained as analogous to the effect of oxygen compounds (see fig. 19, II). In other words, a chlorine atom in ethylene dichloride, like oxygen in alcohols, ethers, etc., apparently can serve as a proton acceptor with primary or secondary ammonium salts, with the formation of a hydrogen bridge between $\mathrm{N}$ and $\mathrm{Cl}$. As a result, the dimeric form of the salt gives place to the monomer. An additional effect of ethylene dichloride is to shift the absorption band approximately $30 \mathrm{~m} \mu$ toward the infrared. A bathochromic effect upon dyes is often observed as the dielectric 


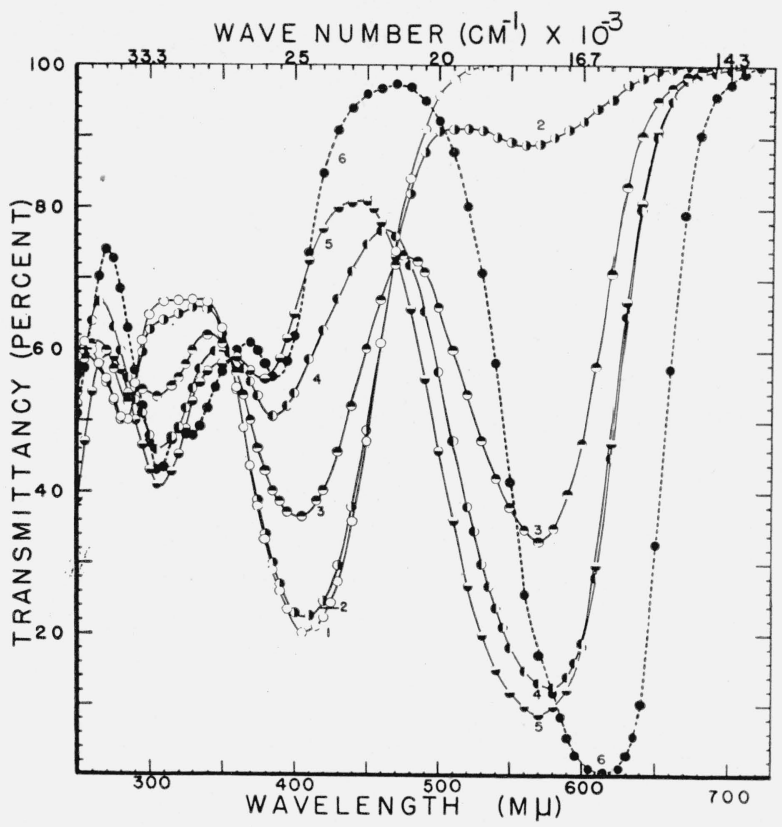

Figure 24. (1 to 5) Transmittancy curves for bromphthalein magenta $E, 5 \times 10^{-5} M$ in ethylene dichloride, in 5 -mm cell, with 1 molar equivalent of the following bases.

(1) None; (2) $n$-butylamine; (3) di- $n$-butylamine; (4) triethylamine; (5)1,3-di-o-tolylguanidine; (6) transmittancy curve for tetraethylammonium salt of bromphthalein magenta $\mathrm{E}, 5 \times 10^{-5} \mathrm{M}$ in ethylene dichloride, 5 -mm cell.

constant of the solvent is increased, and the dielectric constant of ethylene dichloride (10.24) is appreciably greater than that of benzene (2.27). Whether ethylene dichloride exerts its effect through dipole orientation [76] or in some other manner is debatable. The apparent effect of the solvent, however, is to reduce the extent of interaction of the indicator anion with the various cations, thus increasing the degeneracy of the resonance structures of the anion.

The reactions shown by curves 2 through 5 were incomplete, as only one molar equivalent of amine had been added to the indicator. The transmittancies at $570 \mathrm{~m} \mu$ are therefore a measure of the relative strengths of the bases. It is evident that these basicities fall in the same order as in benzene solution.

Other solvents.-Discussion of solvent effects.Qualitative observations of the color of brom- phthalein magenta and of its salts with primary, secondary, and tertiary amines in solvents of different types are summarized in table 7 . For the indicator and for these three types of salts, a solvent effect is plainly evident. In the table the color of the quaternary ammonium salt is also stated for a number of solvents; for this type of compound a solvent effect is absent or slight. Conclusions drawn from visual tests are necessarily tentative because spectrophotometric measurements often reveal differences that are not evident to the eye. It is also possible that traces of impurities were present in some of the solvents. Furthermore, as stated in section IV-8, in oxygen-containing solvents, the color is dependent upon the concentration of the indicator and base. Nevertheless, for bromphthalein magenta and for its quaternary or tertiary ammonium salts, the following simple explanations of solvent effects are possible: 
TABLE 7.-Effect of solvent and added base upon the color of bromphthalein magenta ${ }^{\mathrm{a}}$

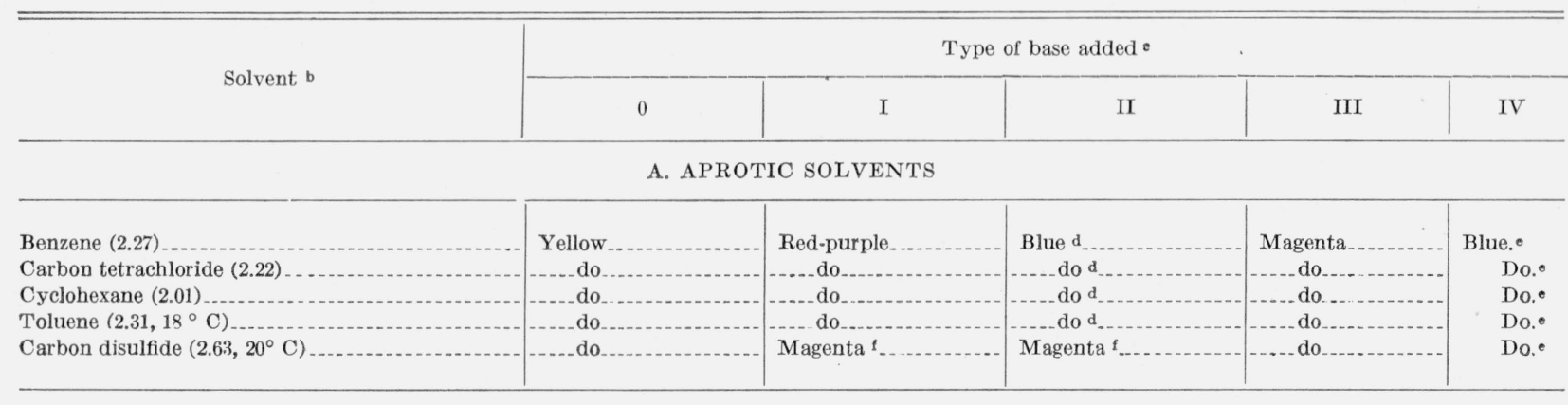

\section{B. PARTLY HALOGENATED HYDROCARBONS}

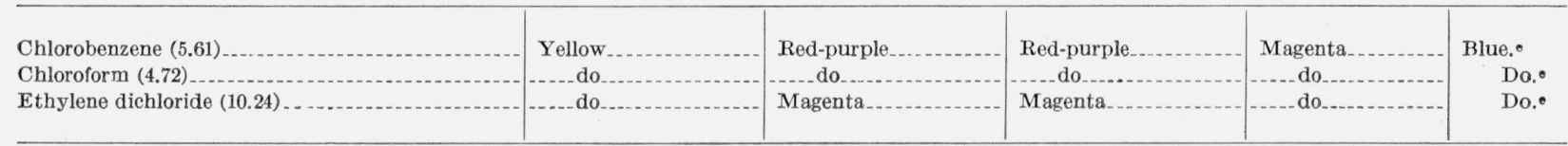

C. ETHERS

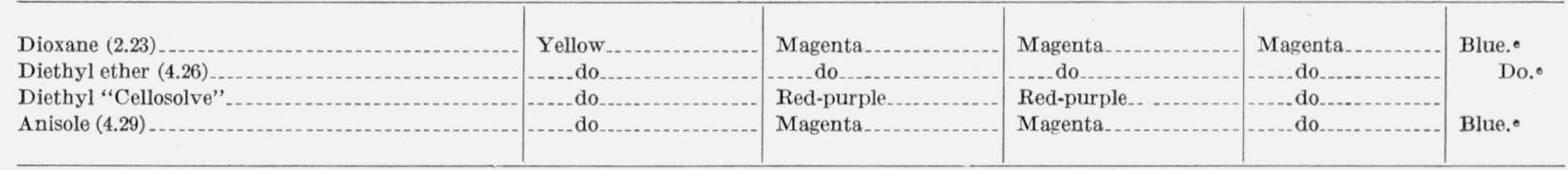

D. ESTERS

Ethyl acetate $\left(4.5,18^{\circ} \mathrm{C}\right)$

Butyl stearate $\left(3.12,30^{\circ} \mathrm{C}\right) \mathrm{g}$

Dibutyl sebacate $\left(4.55,30^{\circ} \mathrm{C}\right) \mathrm{g}$

Methyl benzoate $\left(6.9,20^{\circ} \mathrm{C}\right)$.

Benzyl benzoąte $\left(4.8,20^{\circ} \mathrm{C}\right)$

Tributyl phosphate $\left(7.97,30^{\circ} \mathrm{C}\right) \mathrm{g}$

\begin{tabular}{l} 
Red-purple_....... \\
Magenta \\
Red-purple \\
\hline Blue
\end{tabular}

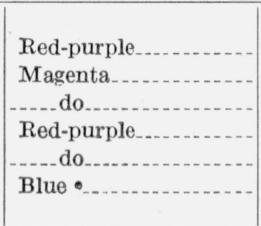

\section{E. KETONES}

Acetone (20.88)

Methyl isobutyl ketone.

Acetonylacetone

Cyclohexanone $\left(18.2,20^{\circ} \mathrm{C}\right)$.

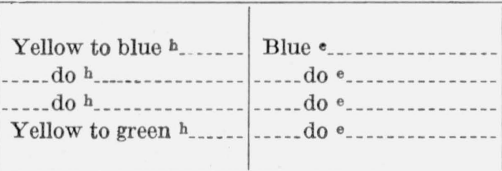

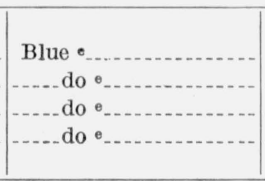

\begin{tabular}{|} 
Magenta $\ldots \ldots$ \\
\hline do \\
\hline Blue \\
\hline do \\
\hline do
\end{tabular}

\section{F. ALCOHOLS :}

Ethyl alcohol $\left(23.2,30^{\circ} \mathrm{C}\right)$

$n$-Propyl alcohol $\left(21.2,21.9^{\circ} \mathrm{C}\right)$

$n$-Butyl alcohol $\left(18.4,20.5^{\circ} \mathrm{C}\right)$

Heptanol-2..

Octyl alcohol (2-ethylhexanol)

Heptadecanol (3,9-diethyltridecanol-6)

Cyclohexanol $\left(8.5,18^{\circ} \mathrm{C}\right)$

Benzyl alcohol $\left(13.6,21.5^{\circ} \mathrm{C}\right)$

2.Methyl-2,3-pentanediol $\left(24.4,30^{\circ} \mathrm{C}\right)$

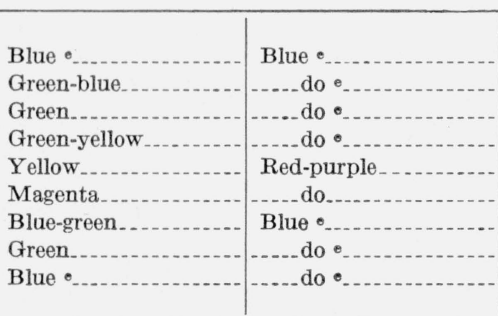

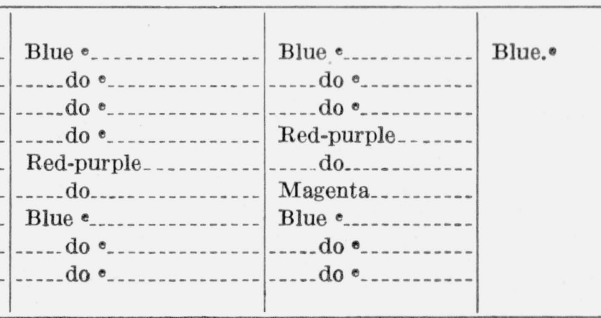

See footnotes at end of taple. 


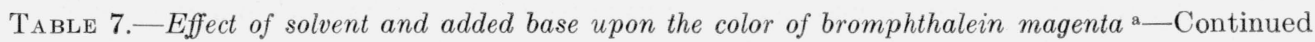

\begin{tabular}{|c|c|c|c|c|c|}
\hline \multirow{2}{*}{ Solvent b } & \multicolumn{5}{|c|}{ Type of base added 0} \\
\hline & 0 & I & II & III & IV \\
\hline
\end{tabular}

G. MISCELLANEOUS

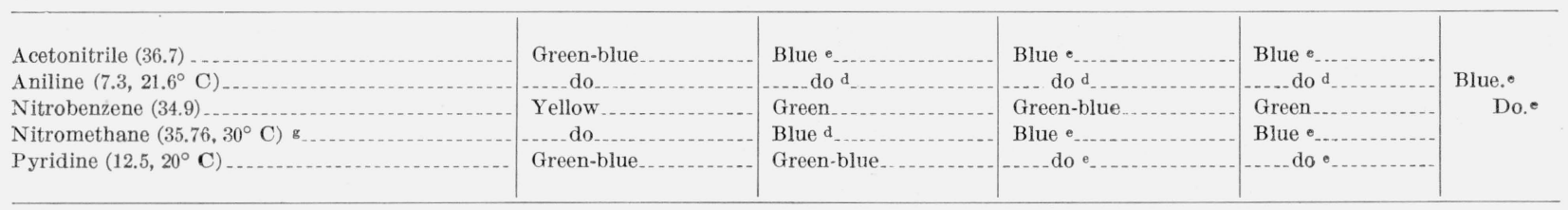

a Bromphthalein magenta B was used in these tests.

b Numbers in parentheses are dielectric constants, at $25^{\circ} \mathrm{C}$ unless otherwise indicated. Values were taken from the literature [92,93], except as noted.

- Key to bases used: $\mathrm{O}=$ no base; $\mathrm{I}=\mathrm{C}_{8} \mathrm{H}_{17} \mathrm{NH}_{2} ; \mathrm{II}=\left(n-\mathrm{C}_{4} \mathrm{H}_{9}\right)_{2} \mathrm{NH} ; \mathrm{III}=\left(\mathrm{C}_{2} \mathrm{H}_{5}\right)_{3} \mathrm{~N} ; \mathrm{IV}=\left(r-\mathrm{C}_{4} \mathrm{H}_{8}\right)_{4} \mathrm{~N}-$ salt of bromphthalein magenta $\mathrm{B}$.

d Purple-blue.

- Blue, without purple tinge.

f Base reacted to some extent with solvent.

g Values were obtained by A. A. Maryott and C. G. Malmberg of the Bureau for high-grade commercial solvent.

b Yellow in concentrated solution, bluer upon dilution.

in concentrated solutions, the color shifts toward yellow.

(1) Bromphthalein magenta.-As stated in the introduction, it is fairly well established that ionization of most acids is dependent chiefly upon the presence of proton-attracting molecules. For example, Kraus observed that hydrogen chloride is a very weak electrolyte in nitrobenzene, in spite of the rather high dielectric constant of this solvent [57a]. On the other hand, perchloric acid is a rather strong electrolyte in nitrobenzene. There is evidently a considerable difference in the affinity of the chloride and perchlorate ions for protons. As pointed out in the introduction, this difference is masked in aqueous solutions, as protons of both acids unite with the solvent to give $\mathrm{H}_{3} \mathrm{O}^{+}$ions. From the behavior of the acid, bromphthalein magenta, it is to be inferred that the affinity of its anion for a proton is high. In solvents of classes $A, B$, and $C$, there is no evidence of ionization, whereas solvents that have a high affinity for protons, such as alcohols and pyridine, compete with the anion of the acid, producing. solvated hydrogen ions and liberating the blue anions. Ionization of the indicator acid is incomplete, however, except at very high dilutions. The peculiar behavior shown by heptadecanol (3,9diethyltridecanol-6) in producing a magenta tone rather than a blue color was also observed for tetradecanol (7-ethyl-2-methylundecanol-4). It is noteworthy that both compounds have two large hydrocarbon radicals adjacent to the $-\mathrm{OH}$ group. The following explanation for the magenta color is suggested: The hydrogen in the $-\mathrm{OH}$ group of the indicator acid forms a bridge to the oxygen in the $-\mathrm{OH}$ group of the alcohol, with the alcohol taking the role of a base. The hydrogen bridge is shielded by the hydrocarbon radicals in the alcohol, so that further solvation of the proton is impeded. It seems likely that a completely solvated proton forms a hydrogen bridge between two molecules of solvent, and complete solvation by either of these alcohols may be prevented by steric factors.

(2) Quaternary ammonium salts.-For these compounds, the attraction between the cation and the anion is Coulombic in nature and probably has little or no influence upon the electronic system of the anion. On the basis of the resonance hypothesis, the degeneracy of the structures contributing to the resonance of the anion may be considered essentially complete. Consequently, the extent to which a quaternary ammonium salt exists as free ions, ion-pairs, or other aggregates is not measurable spectrophotometrically.

(3) Tertiary ammonium salts.-In section IV-4, the behavior of tertiary ammonium salts in benzene was considered and a hydrogen bridge between the cation and anion was postulated. This is the explanation that Kraus advanced to account for the low dissociation constants of most salts of this type in solvents having no affinity for the proton. He attributed the leveling effect of pyridine upon the dissociation of ammonium salts to interaction of the proton of the cation with a pyridine molecule. In the absence of a proton- 
attracting solvent, the proton of the cation interacts strongly with the anion of the salt [57]. The behavior of trialkylammonium salts of bromphthalein magenta may be given a similar interpretation. That is, a magenta color indicates interaction between the proton of the trialkylammonium ion and the anion; a blue color is evidence that the proton of the cation has interacted with a molecule of the solvent. If the conductance were measured, a negligible value for the magenta solution and a measurable value for the blue solution would be predicted. In analogy with conclusions reached by Brooker in studies of other dyes [77], one may conclude that the degeneracy of the resonance structures of the anion in a trialkylammonium salt of bromphthalein magenta is incomplete, as shown by the fact that spectral absorption occurs at shorter wavelengths than that of tetraalkylammonium salts. It is to be recalled that salts of symmetrical diarylguanidines and triphenylguanidine behave in the same way as trialkylammonium salts of bromphthalein magenta.

(4) Secondary ammonium salts.-Before discussion of dialkylammonium salts of bromphthalein magenta, the experimental data given in table 7 and in earlier sections of the paper may be summarized:

(a) In hydrocarbons and in carbon tetrachloride, the color is purple-blue; (b) addition of small amounts of alcohol or other oxygen-containing solvents produces a change in color from purpleblue to magenta; (c) in partly halogenated hydrocarbons, ethers, and most esters, the color is magenta or red-purple; (d) in solvents of high proton-affinity, the color is blue. This is a different color, visually and spectrophotometrically, from the purple-blue of solutions in solvents of class $A$.

An explanation for observations (a), (b), and (c) was offered in section IV-8, and on page 253 . The blue color of solutions in solvents of high proton-affinity (observation (d)) resembles that exhibited by tertiary-ammonium salts in these solvents and is to be accounted for in a similar way.

In addition to the effects discussed in the preceding paragraph, note should be made of the fact that the purple-blue of solutions in benzene is changed to magenta, not only by compounds that contain oxygen or halogen, but also by other types of solvents, including acetonitrile, aniline, and nitrobenzene. In studying the deuteron-attracting properties of liquids by infrared spectroscopy, Gordy and his collaborators [78, 79] measured the shift of the OD band of heavy methyl alcohol, $\mathrm{CH}_{3} \mathrm{OD}$, or heavy water, $\mathrm{D}_{2} \mathrm{O}$. Benzene was used as reference solvent for $\mathrm{CH}_{3} \mathrm{OD}$ or $\mathrm{D}_{2} \mathrm{O}$. Nitrobenzene was found to produce a small but measurable shift, and acetonitrile, oxygen compounds, and amines produced a greater shift, in the order named. The shift produced in the OD band was greater for $\mathrm{D}_{2} \mathrm{O}$ than for $\mathrm{CH}_{3} \mathrm{OD}$. The $\mathrm{OD}$ band in $\mathrm{CH}_{3} \mathrm{OD}$ was not shifted by bromobenzene. The conclusion was reached that nitrobenzene can be linked through deuterium to the oxygen of $\mathrm{D}_{2} \mathrm{O}$ or $\mathrm{CH}_{3} \mathrm{OD}$. Moreover, an intramolecular hydrogen bridge in $o$-nitrophenol is generally postulated to account for differences in the behavior of this compound and its $m$ - and $p$-isomers.

From the experimental data presented in this paper, it is evident that in a dialkylammonium salt of bromphthalein magenta, the cation behaves as a strong proton donor. When the nitrogen of a dialkylamine interacts with the proton of bromphthalein magenta, the proton originally attached to the amine nitrogen evidently becomes more loosely held, so that it interacts readily with oxygen, halogen, or nitrogen atoms of the same or other molecules. ${ }^{35}$ As a result, the secondary ammonium salt of bromphthalein magenta becomes either dimerized or solvated, depending upon whether the medium is an inert solvent or contains compounds of high protonaffinity. The salt can be solvated by bromobenzene and chlorobenzene, as well as by the solvents found by Gordy to exhibit bonding with $\mathrm{CH}_{3} \mathrm{OD}$.

The solvent, tri- $n$-butyl phosphate, deserves special comment. From table 7, class D, it is evident that this compound possesses a higher solvating capacity than the usual types of esters. It is therefore interesting to note that it causes a much larger shift in the $\mathrm{OD}$ band of $\mathrm{CH}_{3} \mathrm{OD}$ than other esters [79]. Moreover, the esters of phosphoric acid are exceptionally good solvents for monofluorodichloromethane [80]. In all these cases, the observations can be attributed to the strong proton-affinity of the $\mathrm{P}=\mathrm{O}$ group.

\footnotetext{
${ }_{35}$ As a corollary, it might be postulated that a mono- or dialkylamine should show greater reactivity with an acid (in an aprotic solvent) after the addition of a proton-accepting substance.
} 
From a comparison of curves 2, 3, and 4 in figure 21 it may be concluded that in the dimeric form of a dialkylammonium salt of bromphthalein magenta, the degeneracy of the resonance structures is almost as great as in a tetraalkylammonium salt [77]. Dicyclohexylguanidine appears to resemble secondary amines in its behavior rather than tertiary amines and di- or triarylguanidines.

(5) Monoalkylammonium salts.-As stated in section IV-4, monoalkylammonium salts of bromphthalein magenta appear to exist partly in the monomeric and partly in the dimeric condition. The limited stability of the dimeric form may be explained in several ways:

(a) A monoalkylamine does not interact so completely with the proton of the acid and consequently the cation of the salt has weaker protondonor properties; (b) as the cation possesses two protons, neither proton is so readily donated as the single proton of a dialkylammonium salt; (c) the dimer of a monoalkylammonium salt of bromphthalein magenta has lower symmetry than the dimer of a dialkylammonium salt; (d) a monoalkylamine has sufficiently strong proton-acceptor properties to become coordinated with a mono- or dialkylammonium ion.

Interpretation of the behavior of tetrahydroquinoline involves similar factors, as well as a possible effect of the adjacent benzene ring.

In the above discussion, the possible role of the dielectric constant of the solvent has been to a large extent ignored. As the attraction between the anion and incompletely substituted ammonium ions is partly Coulombic in nature [57], the dielectric constant undoubtedly has some effect. However, the general pattern of behavior of bromphthalein magenta and its salts can be interpreted satisfactorily from a consideration of the proton-affinity of the solvent, but not from a consideration of its dielectric constant. Gordy has pointed out that there is no correlation evident in the dipole moments of solvents and the strengths of the bonds formed with $\mathrm{CH}_{3} \mathrm{OD}$ [79].

\section{Sulfonephthalein Analogs and Other Indicators}

Further brief mention may be made of the fact that the peculiarities in behavior described above for bromphthalein magenta are not characteristic of this indicator alone. For example, differences in the colors produced by the addition of secondary and tertiary amines to the indicator in benzene solution are shown by several other indicators. These include the following sulfonephthaleins: bromcresol green, bromcresol purple, bromphenol blue, chlorphenol blue, bromchlorphenol blue, iodophenol blue, and tetrabromphenol blue. Ethyl eosin (the ethyl ester of eosin free acid) and fluorescent blue show characteristic differences in their behavior with secondary and tertiary amines that are quite marked spectrophotometrically, though less readily detected by the unaided eye [43]. These indicators have a related structure. With the exception of fluorescent blue, they may be regarded as derivatives of benzaurin (fig. 4), with one or more halogen atoms ortho to the phenol and quinoid oxygens.

In inert solvents, bromphthalein magenta is superior to the other indicators listed here, on account of its greater solubility as well as the brilliant and sensitive color changes that it undergoes in reactions with aliphatic amines. Furthermore, being a monobasic acid, its reactions can be studied more readily than those of the sulfonephthalein indicators, which are dibasic acids. As is to be expected, the carbethoxy group, $-\mathrm{COOC}_{2} \mathrm{H}_{5}$, undergoes hydrolysis by strong aqueous acid or alkali and conversion to tetrabromophenolphthalein results. In addition, bromphthalein magenta is almost insoluble in water, as by intention it lacks the water-solubilizing sulfonic acid group that is present in the sulfonephthaleins. In short, an indicator must be tailored to fit the medium in which it is to be used. The sulfonephthaleins are excellent indicators for aqueous media, but unsuited for use in hydrocarbons, whereas the reverse is true for bromphthalein magenta.

Bromphthalein magenta, the analog of bromphenol blue, is only one of a series of indicators that should prove useful in organic media. The analogs of bromcresol green and other sulfonephthaleins would undoubtedly be valuable. Other derivatives of benzaurin, not necessarily containing the carbethoxy group, are possibilities. For example, $\alpha$-naphtholbenzein is soluble in benzene and, being less acidic than bromphthalein magenta, is more satisfactory for some purposes.

The progressive solvation of incompletely alkylated ammonium ions, so readily observed for the salts of bromphthalein magenta and the other halogenated indicators referred to above, because of the striking color changes that accompany sol- 
vation, undoubtedly occurs in the case of alkylammonium salts of other acids. This study of bromphthalein magenta therefore serves not only to clarify its behavior and that of its analogs, but to aid in interpreting the behavior of alkylammonium salts in general.

\section{Acid-base Titrations in Organic Solvents}

For many years, it was usually assumed that acid-base reactions could not occur in a non-aqueous medium. Hantzsch and numerous others showed that this supposition is incorrect. Moreover, the feasibility of performing quantitative colorimetric titrations in inert solvents has been reported by several investigators. The progress made in this field may be reviewed briefly.

Aminometric titrations.-In 1903, Vorländer announced the successful titration of aniline with a dilute solution of anhydrous hydrogen chloride in benzene, with methyl yellow ( $p$-dimethylaminoazobenzene) as indicator [81]. At that time, Vorländer was interested in correlation of the color and structural changes of the indicator rather than in titrimetric analyses. Thirty years passed before he turned to possible analytical applica. tions of the titration, for which he coined the name "aminometry" or "alkalimetry without hydroxyl ions" [82, 83]. Investigations showed that with chloroform or carbon tetrachloride in place of benzene as the solvent, the color change was sharper. Chloroform was best because of its solvent power and could be used alone or in mixtures with benzene or toluene. Benzenesulfonic acid and other anhydrous sulfonic acids were as satisfactory as hydrogen chloride, and changed titer less rapidly. The acid could be standardized against aqueous alkali or against tribenzylamine in chloroform.

A number of aromatic and aliphatic amines not satisfactorily titratable in aqueous or alcoholic media could be titrated with results ranging from less than 1 percent to 2 percent below the theoretical values. Volatile amines were dissolved in an excess of the acid and back-titrated with tribenzylamine solution. Brucine, one of the alkaloids that titrated successfully, gave equally good results for the anhydrous and the hydrated forms. With precautions, both amino groups of certain diacidic alkaloids could be titrated. Picric acid was not usable for lack of a suitable indicator.
A short time later, Dietzel and Paul became interested in Vorländer's aminometric procedure as a means of simplifying pharmacopoeial methods for the analysis of alkaloids and other drugs and their preparations and made further tests of its applicability to primary, secondary, and tertiary aliphatic and aromatic amines [84]. In preliminary experiments, the titration vessel was protected from access of atmospheric moisture, but this precaution was later found unnecessary. Anhydrous $p$-toluenesulfonic acid in chloroform proved to be the most satisfactory standard solution. Hexamethylenetetramine, which acts as a monoacid base, was equally as good as tribenzylamine for determining the titer of the acid, was cheaper and was more easily purified. The base concerned was weighed exactly into a beaker and titrated with $0.05-M p$-toluenesulfonic acid in chloroform, with the addition of 5 drops of 0.05 -percent methyl yellow in chloroform until the color changed sharply to red. Alkaloids and drugs that could be titrated with an accuracy of \pm 1 percent included atropine, cinchonine, papaverine, narcotine, codeine, strychnine, and ephedrine. The addition of about 1 percent of chemically pure phenol by volume led to a sharper color change. Morphine, although insoluble in chloroform alone, dissolved in 1:3 phenol-chloroform and was titrated exactly. Accurate results were obtained for nonvolatile aliphatic amines and for aromatic amines of basicity comparable to that of aniline. Any halogen-containing solvent, such as tetrachloroethane or methylene chloride, could be substituted for chloroform.

Khairt [85] used the procedure of Dietzel and Paul in the assay of atropine, lobeline, and harmine. Preliminary experiments with various types of amines confirmed the superiority of $p$-toluenesulfonic acid to other acids. A $0.05-M$ solution of the acid in chloroform gave better results than a less concentrated solution. With bromphenol blue as the indicator, the color change was not sharp in many cases. Quinine was found to titrate as a monoacidic base in the presence of bromphenol blue and as a diacidic base in the presence of methyl yellow. Tribenzylamine was not available for standardization of the acid, and codeine recrystallized from alcohol and dried to constant weight at $100^{\circ} \mathrm{C}$ proved a satisfactory substitute. The results were much better when chloroform was used as the solvent than when the 
titrations were performed in aqueous or alcoholic media.

Trautner and coworkers have made further applications of Vorländer's aminometric titration to the analysis of pharmaceutical preparations and plant materials for their alkaloid content, following the modifications introduced by Dietzel and Paul [86, 87]. $\quad$-Toluenesulfonic acid suitable for the analyses is obtained by dehydrating the purified monohydrate. This is accomplished by heating the monohydrate at $10-\mathrm{mm}$ pressure and under a current of air dried with phosphorus pentoxide for 1 hour to $120^{\circ} \mathrm{C}$. The product is a grayish crystalline mass that melts at $105^{\circ} \mathrm{C}$, is not very hygroscopic, and can be kept in a wellstoppered bottle [86]. Nicotine, which is slightly more than monoacidic toward $p$-toluenesulfonic acid, reacts as a diacidic base with picric acid. Precipitation of the monopicrate is prevented by warming the solution to about $40^{\circ} \mathrm{C}$ [87].

In nearly all of the experiments cited, the indicator used was methyl yellow. Methyl yellow is probably a stronger base than some of the bases titrated, and this may account for the fact that in a number of cases less acid was consumed than that equivalent to the known content of base. The weaker base, benzeneazodiphenylamine, dissolves readily in inert solvents and changes color upon the addition of sulfonic acids. Its use in place of methyl yellow might yield more accurate results in titrations of some of the weaker organic bases. There are probably still other indicator dyes, commercially available or readily synthesized, which would extend the usefulness of the method. The role of phenol in sharpening the methyl yellow endpoint is not explained and de. serves investigation. $p$-Toluenesulfonic acid appears to be the most satisfactory reference acid for most purposes, but at the present time it is necessary for each worker to purify and dehydrate the commercial product. A wider recognition of the feasibility of such titrations and commercial availability of anhydrous strong acids and suitable indicators should lead to other analytical applications.

Titrations of weak organic acids in mixtures of absolute alcohols with aprotic solvents.-About 35 years ago, Folin and coworkers directed attention to the successful titration of weak acids, such as benzoic and stearic acids, dissolved in benzene, toluene, chloroform, or carbon tetrachloride, with sodium ethylate or amylate in the corresponding absolute alcohol as the base, and any one of several indicators, preferably phenolphthalein. Although the conductivities of their solutions were found to be extremely low, the reactions were instantaneous and the behavior of the indicator essentially the same as in aqueous solutions [88 to 90]. This work did not receive wide attention, as it antedated the Brönsted-Lowry and Lewis theories and the results reported violated the theory then current, that dissociation into ions is a prerequisite in chemical reactions.

The Folin method is excellent for determination of the acid content of impure solvents, such as ligroin and turpentines, except in the case of very old turpentines in which extremely weak acids, possibly peroxides, show a buffering effect upon the endpoint [91]. This might be overcome by the use of an indicator of higher acidity than phenolphthalein. Bromphthalein magenta can be used in the titration of relatively strong acids, such as trichloroacetic acid, but is too strong an acid to be used in the titration of acetic and benzoic acids. Dicyclohexylguanidine, $0.1 M$ in absolute ethyl alcohol, is a convenient substitute for sodium ethylate and gives exact results in the titration of trichloroacetic acid, picric acid, or benzoic acid in benzene, with $\alpha$-naphtholbenzein as indicator but not with phenolphthalein. Bromphthalein magenta is slightly superior to $\alpha$-naphtholbenzein in the titration of picric acid [91].

The Folin procedure was adapted by Lavine and Toennies to the differential titration of acetic and perchloric acids in acetonitrile, with methyl alcoholic sodium methylate as the base. Thymol blue was used as the indicator, changing from pink to yellow when the perchloric acid was neutralized, and from yellow to blue at the acetic acid endpoint [92].

Titrations in aprotic solvents with bromphthalein magenta as indicator.-As shown earlier in this paper, 1,3-diphenyl-, di-o-tolyl- and dicyclohexylguanidine appear to be stronger bases in benzene than any of the aliphatic amines, with increasing. strength in the order named. Dicyclohexylguanidine is too difficultly soluble in benzene for practical use, although it produces a color change not only with bromphthalein magenta but with the weaker acid, $\alpha$-naphtholbenzein. Di-o-tolyl- and diphenylguanidine react readily with bromphthalein magenta but not with $\alpha$-naphtholbenzein. As 
bromphthalein magenta is a relatively strong acid, it cannot be used in the titration of acids as weak as benzoic and acetic acids. As pointed out earlier in the discussion, there is need for a strong guanidine derivative or other organic base that is readily soluble in benzene. Dicyclohexylguanidine is fairly soluble in chloroform but reacts with the solvent, particularly upon warming. This reaction is not sufficiently rapid at room temperature to prevent excellent titrations with acetic acid, o-chlorophenoxyacetic acid, and picric acid, with $\alpha$-naphtholbenzein as the indicator [91].

A series of experimental titrations with benzene as solvent and a 0.1-percent solution of bromphthalein magenta in benzene as the indicator is summarized in table 8 . The total volume was approximately $30 \mathrm{ml}$, and the volume of acid or base was less than $10 \mathrm{ml}$ in all cases and as little as 1 to $2 \mathrm{ml}$ in some instances. Moreover, in three

TABLE 8.-Acid-base titrations in benzene with bromphthalein magenta as indicator

\begin{tabular}{|c|c|c|}
\hline Reference base & Acid & $\begin{array}{l}\text { Molarity found } \\
\text { for acid }\end{array}$ \\
\hline $\begin{array}{l}\text { Diphenylguanidine, } \\
0.0100 \mathrm{M.}^{\mathrm{s}}\end{array}$ & $\begin{array}{l}d \text {-Camphorsulfonic acid (Rey- } \\
\text { chler), approximately } 0.005 \\
\text { M. }\end{array}$ & $\begin{array}{l}0.00527, \quad 0.00529 \\
0.00528 \text {. }\end{array}$ \\
\hline Reference acid & Base & $\begin{array}{l}\text { Molarity found } \\
\text { for base }\end{array}$ \\
\hline $\begin{array}{l}d \text {-Camphorsulfonic } \\
\text { acid, } 0.00528 M .^{\text {b }}\end{array}$ & $\begin{array}{l}\text { Impure dicyclohexylguani- } \\
\text { dine, approximately } 0.0025 \\
M \text {. }\end{array}$ & $0.00238, \quad 0.00237$ \\
\hline $\begin{array}{l}\text { Trichloroacetic acid, } \\
\quad 0.106 \mathrm{M} \text {. }^{\circ}\end{array}$ & $\begin{array}{l}\text { Di-o-tolylguanidine, approxi- } \\
\text { mately } 0.05 \mathrm{M} \text {. }\end{array}$ & $\begin{array}{l}0.0484, \quad 0.0484 \\
0.0487 \text {. }\end{array}$ \\
\hline Picric acid $0.0100 M^{\mathrm{a}}$ & $\begin{array}{l}\text { Di-o-tolylguanidine, approxi- } \\
\text { mately } 0.01 \quad M .^{\mathrm{d}}\end{array}$ & $\begin{array}{l}0.00970, \quad 0.00972, \\
0.00972 .\end{array}$ \\
\hline $\begin{array}{l}\text { Trichloroacetic a ci d, } \\
0.106 \mathrm{M} \text {. }\end{array}$ & $\begin{array}{l}\text { Triethylamine, approximately } \\
0.1 \mathrm{M}^{\mathrm{e}}\end{array}$ & ${ }^{1} 0.0942, \quad 0.0944$ \\
\hline $\begin{array}{l}\text { Trichloroacetic a cid, } \\
0.0106 M_{. \mathrm{g}}\end{array}$ & $\begin{array}{l}\text { Triethylamine, approximately } \\
0.01 M_{.}^{\mathrm{h}}\end{array}$ & $\begin{array}{l}0.00935, \quad 0.00935, \\
0.00936 \text {. }\end{array}$ \\
\hline Picric acid, $0.0100 M^{\mathrm{s}}$ & $\ldots$ do $b_{\ldots} \ldots \ldots \ldots \ldots$ & $\begin{array}{r}0.00962, \quad 0.00962 \\
0.00960,0.00962 .\end{array}$ \\
\hline $\begin{array}{l}\text { Trichloroacetic a cid, } \\
0.106 \mathrm{M} \text {. }^{\circ}\end{array}$ & $\begin{array}{l}\text { Piperidine, approximately } \\
\quad 0.1 M^{e}\end{array}$ & f $0.0957,0.0953$ \\
\hline Picric acid, $0.100 M^{\mathrm{a}}$ & do ${ }^{\circ}$ & $\begin{array}{rr}\text { i } 0.0982, & 0.0990 \\
0.0977 . & \end{array}$ \\
\hline
\end{tabular}

a The solid was assumed to be 100 percent pure.

b Standardized against diphenylguanidine in benzene.

- Standardized against sodium ethylate in absolute ethyl alcohol with phenolphthalein as indicator: the sodium ethylate was standardized against benzoic acid with the same indicator.

d Diluted from the approximately $0.05-M$ solution.

- Would be $0.100 M$ if no impurities were present.

${ }^{\prime}$ A cloud formed during the titration. Methyl yellow did not give a sharp color change.

$\mathrm{g}$ Diluted from the $0.106-M$ solution.

h Diluted from the approximately $0.1-M$ solution.

i Picrate hegan to precipitate out. of the titrations the concentrations of the acid and the base differed by a factor of two. The endpoints were very sharp, vivid, and reproducible except in the titration of piperidine with trichloroacetic acid, where the formation of a cloud caused slightly lower precision.

From inspection of the table it can be noted that trichloroacetic acid and picric acid, used at different concentrations, gave the same value in the titration of ditolylguanidine. With triethylamine or piperidine as the base, trichloroacetic acid gave lower values than picric acid. From the standpoint of reproducibility and vividness of the color change, the results are very satisfactory.

Diphenylguanidine was used by Kilpatrick for the quantitative estimation of the very strong acid, perchloric acid, in acetonitrile, with methyl yellow, benzoyl auramine, or thymol blue as the indicator, but an organic base sufficiently strong for the estimation of acetic acid in this solvent was lacking [45]. It should be possible, after further investigations, to obtain strong acids and bases and a series of indicators that will permit the quantitative titration of weak bases and weak acids in the various aprotic solvents.

\section{Summary. Applications of Brom- phthalein Magenta}

The differentiating reaction of bromphthalein magenta with various classes of organic bases in inert solvents, and its uses for quantitative comparisons of basic strengths and for certain acidbase titrations were described above. An additional application is in the measurement of the relative strengths of organic acids in inert solvents. Studies are in progress in this laboratory in which the strength of an acid is measured by the extent of its competition with bromphthalein magenta for a reference base, such as di-o-tolylguanidine. Preliminary measurements have been made for more than 30 acids, that include acetic and benzoic acids as well as a number of their more highly acidic derivatives. Some of these are too insoluble to be studied in aqueous solutions [91].

Other applications of the indicator to specific analytical problems will doubtless occur to some readers. The description and interpretations given in this paper of the behavior of the indicator acid in different environments will serve as a guide in selecting the proper conditions. As indicated 
in section IV-9, qualitative tests can be made with salts as well as with the corresponding bases.

The experimental data of the authors and the investigations of other workers that have been reviewed in this report point to promising lines of work in the practical and theoretical study of acid-base reactions.

The authors express their indebtedness to $\mathrm{S}$. F. Acree, former chief of section V-10, pH Standards, for suggesting the development of new indicators for the measurement of acidity and basicity in organic solvents, for his interest in the progress of the work, and for his assistance in the designing of various absorption cells used in the spectrophotometric measurements. Grateful acknowledgment is also made to Kenneth D. Fleischer for the chemical analyses, to Wilbur W. Brannon and Fred H. Engler for construction of the stoppered endplates and other portions of the absorption cells, to Mary Ellen Lovelace for assistance in part of the synthetic work, and to the commercial organizations listed in section III of this report for their contributions of bases and solvents used in the investigations.

\section{References}

[1] J. N. Brönsted, Rec. trav. chim. 42, 718 (1923).

[2] J. N. Brönsted, Chem. Rev. 5, 231 (1928).

[3] J. N. Brönsted, Z. angew. Chem. 43, 229 (1930).

[4] J. N. Brönsted, Z. physik. Chem. [A] 169, 52 (1934).

[5] T. M. Lowry, Trans. Faraday Soc. 20, 13 (1924).

[6] A. Hantzsch, Z. Elektrochem. 29, 221 (1923); 30, 194 (1924).

[7] A Hantzsch and W. Voigt, Ber. deut. chem. Ges. 62, 975 (1929).

[8] N. F. Hall and J. B. Conant, J. Am. Chem. Soc. 49, 3047 (1927).

[9] N. F. Hall and T. H. Werner, J. Am. Chem. Soc. 50, 2367 (1928).

[10] N. F. Hall, J. Am. Chem. Soc. 52, 5115 (1930).

[11] E. C. Franklin, The nitrogen system of compounds (Reinhold Publishing Corp., New York, N. Y., 1935).

[12] T. S. Moore, J. Chem. Soc. (London) 91, 1373, 1379 (1907).

[13] W. M. Latimer and W. H. Rodebush, J. Am. Chem. Soc. 42, 1419 (1920).

[14] N. V. Sidgwick, The electronic theory of valency (Oxford Univ. Press, New York, N. Y., 1927).

[15] M. L. Huggins, J. Org. Chem. 1, 407 (1936).

[16] E. N. Lassettre, Chem. Rev. 20, 258 (1937).

[17] L. Pauling, The nature of the chemical bond (Cornell Univ. Press, Ithaca, N. Y., 1st ed., 1939; 2nd ed., 1940).
[18] W. H. Rodebush and A. M. Buswell, J. Phys. Chem. 43, 219 (1939); and other papers by Rodebush, Buswell, and collaborators.

[19] H. Hoyer, Z. Elektrochem. 49, 97 (1943).

[20] W. Gordy, Sci. Monthly 53, 440 (1941); and numerous papers by Gordy and coworkers in J. Chem. Physics and J. Am. Chem. Soc.

[21] G. Briegleb, Z. Elektrochem. 50, 35 (1944).

[22] L. Hunter, Chemistry \& Industry, p. 155 (1944).

[23] G. W. Wheland, The theory of resonance and its application to organic chemistry (John Wiley \& Sons, Inc., New York, N. Y., 1944).

[24] G. N. Lewis, Valence and the structure of atoms and molecules (Chemical Catalog Co., New York, N. Y., 1923).

[25] G. N. Lewis, J. Franklin Inst. 226, 293 (1938).

[26] G. N. Lewis and G. T. Seaborg, J. Am. Chem. Soc. 61, 1886 (1939).

[27] W. F. Luder and S. Zuffanti, The electronic theory of acids and bases (John Wiley \& Sons., Inc., New York, N. Y., 1946). Much of this material is contained in a series of papers by Luder and coauthors in J. Am. Chem. Soc., J. Chem. Education, and Chem. Rev.

[28] P. Walden, Salts, acids, and bases: Electrolytes: Stereochemistry (McGraw-Hill Book Co., Inc., New York, N. Y., 1929).

[29] Acids and bases (J. Chem. Education, Easton, Pa., 1941); More acids and bases (same publisher, 1944). Both are collections of papers by N. F. Hall, L. P. Hammett, W. F. Luder and others, which originally appeared in J. Chem. Education.

[30] I. M. Kolthoff, J. Phys. Chem. 48, 51 (1944).

[31] H. H. Cary and A. O. Beckman, J. Optical Soc. Am. 31, 682 (1941).

[32] A. Baeyer, Liebigs Ann. Chem. 201, 80 (1880).

[33] R. Nietzki and E. Burckhardt, Ber. deut. chem. Ges. 30, 175 (1897).

[34] M. E. Maclean, P. J. Jencks, and S. F. Acree, J. Research NBS 34, 271 (1945) RP1643.

[35] E. Eigenberger, J. prakt. Chem. 130, 75 (1931).

[36] A. Thiel and R. Diehl, Sitzungsber. Ges. Beförderung gesamt. Naturwissenschaften Marburg 62, 472 (1927).

[37] P. Ramart-Lucas, Bull. soc. chim. 8, 865 (1941).

[38] P. Ramart-Lucas, Bull. soc. chim. 10, 279 (1943).

[39] F. Feigl and V. Anger, Mikrochem. Acta 2, 107 (1937); M. Ishidate and T. Sakaguchi, Ber. deut. chem. Ges. 74, 163 (1941).

[40] J. N. Brönsted, Ber. deut. chem. Ges. 61, 2049 (1928).

[41] V. K. LaMer and H. C. Downes, J. Am. Chem. Soc. 55, 1840 (1933).

[42] V. K. LaMer and H. C. Downes, Chem. Rev. 13, 47 (1933).

[43] Marion Maclean Davis, Priscilla J. Schuhmann, and Mary Ellen Lovelace, National Bureau of Standards. Unpublished data.

[44] D. C. Griffiths, J. Chem. Soc. (London), 818 (1938).

[45] M. Kilpatrick, Jr. and M. L. Kilpatrick, Chem. Rev. 13, 131 (1933).

[46] A. Baeyer, Liebigs Ann. Chem. 354, 152 (1907). 
[47] S. F. Acree and E. A. Slagle, Am. Chem. J. 42, 115 (1909).

[48] E. Q. Adams and L. Rosenstein, J. Am. Chem. Soc. 36, 1452 (1914).

[49] C. R. Bury, J. Am. Chem. Soc. 57, 2115 (1935).

[50] G. Schwarzenbach, M. Brandenberger, G. H. Ott, and O. Hagger, Helv. chim. Acta 20, 490 (1937).

[51] B. Cohen, Reprint No. 1131 from the Public Health Reports (Government Printing Office, Washington, D. C., 1927).

[52] L. Pauling, J. Am. Chem. Soc. 58, 94 (1936).

[53] O. R. Wulf, U. Liddel, and S. B. Hendricks, J. Am. Chem. Soc. 58, 2287 (1936).

[54] J. L. Hague, Proc. Am. Soc. Testing Materials 44, 712 (1944).

[55] G. Briegleb, Naturwiss. 30, 436, 469, 506 (1942).

[56] T. S. Moore and T. F. Winmill, J. Chem. Soc. (London) 101, 1635 (1912).

[57a] C. A. Kraus, J. Phys. Chem. 43, 231 (1939).

[57b] C. A. Kraus, Science 90, 281 (1939), and numerous other publications by Kraus and coworkers.

[58] N. L. Cox, C. A. Kraus, and R. M. Fuoss, Trans. Faraday Soc. 31, 749 (1935).

[59] E. C. Lingafelter, J. Am. Chem. Soc. 63, 1999 (1941).

[60] H. C. Brown, H. I. Schlesinger, and S. Z. Cardon, J. Am. Chem. Soc. 64, 325 (1942); H. C. Brown and H. Bartholomay, Jr., J. Chem. Phys. 11, 43 (1943); H. C. Brown, Science 103, 385 (1946); and other papers by H. C. Brown and coworkers.

[61] A. Thiel, A. Dassler, and F. Wülfken, Fortschr. Chem. Physik physik. Chem. 18, (3), 1 (1924).

[62] W. M. Clark, The determination of hydrogens ions, p. 161 (Williams and Wilkins Co., Baltimore, Maryland, 3rd ed., 1928).

[63] I. M. Kolthoff, Acid-base indicators, p. 122 (translation from the 4th German ed. by C. Rosenblum, Macmillan Co., New York, N. Y., 1937).

[64] E. Heuser, Organic chemistry, an advanced treatise, Henry Gilman, Editor-in-Chief, II, 1672 (John Wiley \& Sons, Inc., New York, N. Y., 2nd ed., 1943).

[65] R. M. Fuoss and C. A. Kraus, J. Am. Chem. Soc. 55, 3614 (1933); discussed by H. S. Harned and B. B. Owen, The physical chemistry of electrolytic solutions (American Chemical Society Monograph No. 95, Reinhold Publishing Corp., New York, N. Y., 1943).

[66] W. H. Zachariasen, J. Am. Chem. Soc. 54, 3841 (1932).

[67] B. E. Warren, Chem. Rev. 26, 237 (1940).

[68] L. Hunter and J. A. Marriott, J. Chem. Soc. (London), 777 (1941).
[69] N. Bjerrum, Kgl. Danske Videnskab. Selskab, Math.-fys. Medd. 7,3 (1926).

[70] W. Gordy, J. Am. Chem. Soc. 59, 464 (1937).

[71] A. A. Maryott, National Bureau of Standards. Unpublished data.

[72] T. L. Davis and R. C. Elderfield, J. Am. Chem. Soc. 54, 1499 (1932).

[73] P. Nylén, Tids. Kjemi Bergvesen 18, 48 (1938).

[74] W. R. Dunstan and E. Goulding, J. Chem. Soc. (London) $\mathbf{7 5 ,} 1004$ (1899).

[75] J. Meisenheimer, Liebigs Ann. Chem. 397, 273 (1913).

[76] L. G. S. Brooker and R. H. Sprague, J. Am. Chem. Soc. 63, 3214 (1941).

[77] L. G. S. Brooker, Rev. Modern Phys. 14, 275 (1942), and other papers by Brooker and coworkers.

[78] W. Gordy, J. Chem. Phys. 7, 93 (1939).

[79] W. Gordy and S. C. Stanford, J. Chem. Phys. 8, 170 (1940); also numerous other papers by Gordy and collaborators.

[80] M. J. Copley, G. F. Zellhoefer, and C. S. Marvel, J. Am. Chem. Soc. 60, 2666 (1938).

[81] D. Vorländer, Ber. deut. chem. Ges. 36, 1485 (1903).

[82] D. Vorländer, with J. Fischer and F. Wildner, Ber. deut. chem. Ges. 66, 1789 (1933).

[83] D. Vorländer, Ber. deut. chem. Ges. 67, 145 (1934).

[84] R. Dietzel and W. Paul, Arch. Pharm. 273, 507 (1935); 276, 408 (1938).

[85] G. Ya. Khay̌t, Farmatsiya 8, 26 (1945). For an abstract of the article, see Chem. Abstracts 41, 2206 (1947).

[86] E. M. Trautner and F. H. Shaw, Australian Chem. Inst. J. \& Proc. 12, 232, 405 (1945).

[87] E. M. Trautner and C. E. Neufeld, Australian Chem. Inst. J. \& Proc. 13, 70 (1946).

[88] O. Folin and A. H. Wentworth, J. Biol. Chem. 7, 421 (1910).

[89] O. Folin and F. F. Flanders, J. Am. Chem. Soc. 33, 1622 (1911); 34, 774 (1912).

[90] O. Folin and F. F. Flanders, J. Biol. Chem. 11, 257 (1912).

[91] Marion Maclean Davis and Priscilla J. Schuhmann, National Bureau of Standards. Unpublished data.

[92] T. F. Lavine and G. Toennies, J. Biol. Chem. 101, 727 (1933).

[93] P. Debye and H. Sack, Tables Annuelles de Constantes et Données Numériques XI, Section 22 (Paris, 1937).

[94] L. Hartshorn and A. G. Milligan, Int. Crit. Tables VI, 83 (National Research Council, Washington, D. C., 1929).

Washington, May 5. 1947. 